\title{
Soil and Soil Gas Sampling in Old Ellenton, the SRL Oil Test Site, the Fire Training Area and the Miscellaneous Chemicals Basin
}

by

D. G. Jackson

Westinghouse Savannah River Company

Savannah River Site

Aiken, South Carolina 29808

Universitv of Pittsburgh Applied Research Center

DOE Contract No. $\lambda E-4009-765 \times 00001$

This paper was prepared in connection with work done under the above contract number with the U. S. Department of Energy. By acceptance of this paper, the publisher and/or recipient acknowledges the U.S. Government's right to retain a nonexclusive, royalty-free license in and to any copyright covering this paper, along with the right to reproduce and to authorize others to reproduce all or part of the copyrighted paper. 


\section{DISCLAIMER}

This report was prepared as an account of work sponsored by an agency of the United States Government. Neither the United States Government nor any agency thereof, nor any of their employees, makes any warranty, express or implied, or assumes any legal liability or responsibility for the accuracy, completeness, or usefulness of any information, apparatus, product, or process disclosed, or represents that its use would not infringe privately owned rights. Reference herein to any specific commercial product, process, or service by trade name, trademark, manufacturer, or otherwise does not necessarily constitute or imply its endorsement, recommendation, or favoring by the United States Government or any agency thereof. The views and opinions of authors expressed herein do not necessarily state or reflect those of the United States Government or any agency thereof.

This report has been reproduced directly from the best available copy.

Available to DOE and DOE contractors from the Office of Scientific and Technical Information, P. O. Box 62, Oak Ridge, TN 37831; prices available from (423) 576-8401.

Available to the public from the National Technical Information Service, U. S. Department of Commerce, 5285 Port Royal Road, Springfield, VA 22161. 


\section{DISCLAIMER}

Portions of this document may be illegible electronic image products. Images are produced from the best available original document. 
FINAL REPORT

RFP86-89

"SOIL AND SOIL GAS SAMPLING IN OLD ELLENTON, THE SRL OIL TEST SITE, THE FIRE TRAINING AREA AND THE MISCELLANEOUS CHEMICALS BASIN"

DECEMBER 1986
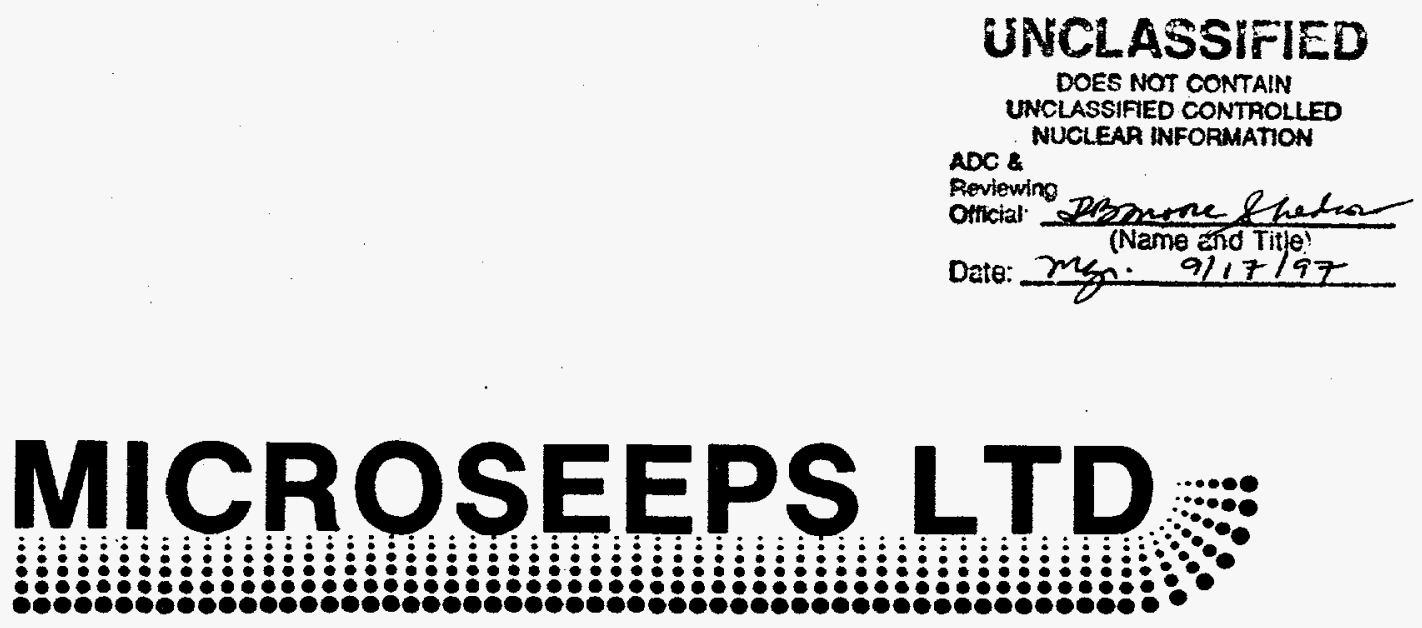

University of Pittsburgh Applied Research Center 220 William Pitt Way Pittsburgh, PA 15238

(412) 826-5245 


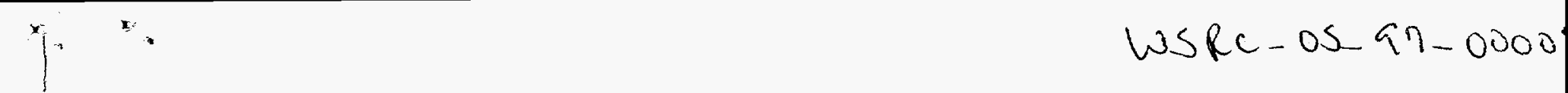

$\begin{array}{lr} & \frac{\text { Page }}{\text { List of Tables }} \\ \text { List of Figures } & i\end{array}$

I. General 1

II. Collection and Analysis of Samples 1

Soil Gas Samples 1

Soil Samples $\quad 2$

Analytical Standards 3

Detection Limits $\quad 5$

Statistics Related to Map Symbols 7

III. Quality Assurance 8

IV. Presentation and Discussion of Data 9

A. Old E1lenton Area 9

Amoco Gasoline Station $\quad 12$

Esso Gasoline Station $\quad 25$

$0: 1$ Company 32

Railroad Depot $\quad 41$

Chevrolet Dealer $\quad 48$

School $\quad 55$

Dry Cleaner $\quad 62$

B. Oil Test Site $\quad 65$

C. Fire Training Area $\quad 81$

D. Miscellaneous Chemicals Basin 94 
LIST OF TABLES

Table

$\underline{\text { Page }}$

1

Minimum Detection Levels

6

2

Amoco Gas Station Soil Gas Concentrations

20

3

Amoco Gas Station Soil Gas Percentages

22

4

Amoco Gas Station Gasoline Range Hydrocarbons

24

5

Esso Gas Station Soil Gas Concentrations

30

6

Esso Gas Station Soil Gas Percentages

31

7

Oil Company Soil Gas Concentrations

37

Oil Company Soil Gas Percentages

39

9

Railroad Depot Soil Gas Concentrations

46

10

Railroad Depot Soil Gas Percentages

47

11 Chevrolet Dealer Soil Gas Concentrations 53

12 Chevrolet Dealer Soil Gas Percentages 54

13 The School Soil Gas Concentrations 60

14 The School Soil Gas Percentages 61

15 The Dry Cleaner Halocarbon Concentrations 64

16 Oil Test Site Soil Gas Concentrations 74

17 Oil Test Site Soil Gas Percentages 76

18 Fire Training Area Soil Gas Concentrations 88

19 Fire Training Area Soil Gas Percentages 90

20 Fire Training Area Gasoline Range Hydrocarbons 92

21 Miscellaneous Chemicals Basin Halocarbon

Concentrations

96 
LIST OF FIGURES

Figure

$\underline{\text { Page }}$

$1 \quad$ 01d Ellenton Area Index Map

11

2 Amoco Gas Station Site Location Map 15

3 Amoco Gas Station - Methane 16

4 Amoco Gas Station - Butane 17

$5 \quad$ Amoco Gas Station - Percent Butane (in C1 - C4) 18

6 Amoco Gas Station - Gasoline Range Hydrocarbon Chromatographic Trace at Site AM-52

7 Esso Gas Station Site Location Map 26

8 Esso Gas Station - Methane 27

9 Esso Gas Station - Butane 28

10 Esso Gas Station - Percent Butane (in C1 - C4) 29

$110 i 1$ Company Site Location Map 33

$120 i 1$ Company Site - Methane 34

$13 \quad 0 i 1$ Company Site - Butane 35

14 0il Company Site - Percent Butane (in Cl - C4) 36

15 Railroad Depot Site Location Map 42

16 Railroad Depot - Methane 43

17 Railroad Depot - Butane 44

18 Railroad Depot - Percent Butane (in C1 - C4) 45

19 Chevrolet Dealer Site Location Map 49

20. Chevrolet Dealer - Methane 50

21 Chevrolet Dealer - Butane 51 
Figure

Page

22 Chevrolet Dealer - Percent Butane (in C1 - C4) 52

23 The School Site Location Map 56

24 The School - Methane 57

25 The School - Butane 58

26 The School - Percent Butane (in C1 - C4) 59

27 The Dry Cleaner Site Location Map 63

$28 \quad 0 i 1$ Test Site Location Map 68

$290 i 1$ Test Site - Methane 69

$30 \quad 0 i 1$ Test Site - Percent Ethylene + Propylene

31 0i1 Test Site - Butane 71

32 0il Test Site - Histogram of Percent Ethylene + Percent Propylene (All Sites) 72

33 0il Test Site - Histogram of Percent Ethylene + Percent Propylene (Control Sites) 73

Dil Test Site - Histogram of Percent Ethylene + Percent Propylene (0il Sites)

$0 i 1$ Test Site - Gasoline Range Hydrocarbon Chromatogram for Site OT-25

0il Test Site - Gasoline Range Hydrocarbon Chromatogram for Site 0T-29 79

$37 \quad 0 i 1$ Test Site - Gasoline Range Hydrocarbon Chromatogram for Site OT-58 80

38 Fire Training Area Site Location Map 83

39 Fire Training Area - Methane 84

40 Fire Training Area - Ethylene + Propylene 85

41 Fire Training Area - Butane 86 
Fire Training Area - Gasoline Range Hydrocarbons

Fire Training Area - Gasoline Range Hydrocarbons Chromatogram for Site FT-47

Miscellaneous Chemical Basin Site Location Map

Miscellaneous Chemical Basin - Tetrachloroethylene

101

Miscellaneous Chemical Basin - Trichloroethylene

102

47

Miscellaneous Chemical Basin - Trichloromethane

Miscellaneous Chemical Basin - cis 1,2 dichloroethylene

Miscellaneous Chemical Basin - trans 1,2dichloroethylene

50 Gasol ine Range Hydrocarbons

$10 \mathrm{ppb}$ standard chromatogram

51 Gasoline Range Hydrocarbons 
FINAL REPORT FOR RFP86-89

"SOIL AND SOIL GAS SAMPLING IN OLD ELLENTON;: THE SRL OIL TEST SITE, THE FIRE TRAINING AREA

"AND THE MISCELLANEOUS CHEMICALS BASIN"

I. General

Presented herein are the soil and soil gas analytical results from RFP86-89 which were carried out by Microseeps, Ltd. at the SRL site. In summary, we have collected and analyzed on-site:

390 soil gas samples for light hydrocarbons $\left(C_{1}-C_{4}\right)$,

85 soil samples for gasoline range $\left(C_{5}-C_{8}\right)$ hydrocarbons, and

136 soil samples for specified chlorinated hydrocarbons

using gas chromatography. The sampling and analytical techniques are described and the data presented in both tabular and mapped formats. The data have already been transmitted to SRL personnel on IBM diskettes and on field maps.

II. Collection and Analysis of Samples

Soil Gas Samples. Samples of soil air were taken from a hole created by driving a 5/8 inch diameter steel rod with a slide hammer. Depth of sampling was mostly at $3 \mathrm{ft}$, although samples from depths ranging from $1 \mathrm{ft}$ to $6 \mathrm{ft}$ were taken at selected sites as a means of establishing depth profiles. After the rod was removed, a probe sampler was inserted into the hole in order to obtain the sample. The probe was equipped with a rubber packer to prevent dilution of the soil gas sample by air. A volume of gas greater than the internal volume of the probe was discarded before a $200 \mathrm{cc}$ soil gas sample was extracted from the 
bottom of the hole using a $250 \mathrm{cc}$ syringe. This sample was injected into a previously evacuated $100 \mathrm{cc}$ serum bottle equipped with a septum. The sample pressure after filling the serum bottle was $\sim 14$ psig. Any leakage, which we have determined to be extremely small, is out of the bottle; thus, no dilution or contamination of the sample is incurred.

Sample analysis was carried out using a gas chromatograph equipped with a flame ionization detector. The chromatograph was designed and built by Microseeps, Ltd. personnel. It provides sensivity in the range of $1 \mathrm{ppb}$ for light hydrocarbons, $\mathrm{C}_{1}$ through $\mathrm{C}_{4}$, and allows rapid analysis time for this suite of compounds. Each soil gas sample was analyzed for methane, ethane, propane, iso-butane, n-butane, ethylene and propylene.

\section{Soil Samples. Soil samples were collected mostly from a depth}

of 18 to 24 inches, except where depth profiles were determined to a depth of 60 inches. Samples were obtained using a $1 \frac{1}{4}$ inch stainless steel pipe which was driven to a depth of 18 inches, removed from the hole and the core discarded. The pipe was again inserted into the hole and a 6 inch sample core obtained. An analogous procedure was followed at other depths.

A portion of this sample core was placed directly into a screw cap sample bottle and sealed with a cap lined with aluminum foil. Each bottle had been washed, rinsed and baked at $90^{\circ} \mathrm{C}$ for a minimum of one hour before use.

Immediately upon return to the laboratory, an $8.0 \pm 0.1$ gram portion of each sample core was obtained using a small soil coring tool, and 
placed in a $22 \mathrm{ml}$ headspace vial. Each vial had similarly been washed, rinsed and baked at $90^{\circ} \mathrm{C}$ before use.

To each vial was added a $5 \mathrm{ml}$ aliquot of a solution consisting of $1000 \mathrm{ml}$ distilled water, $50 \mathrm{gm} \mathrm{Na}_{2} \mathrm{SO}_{4}$ and $1.5 \mathrm{ml}$ conc $\mathrm{H}_{3} \mathrm{PO}_{4}$. The headspace volume of each vial was constant at about $10 \mathrm{ml}$.

Halocarbon and gasoline range hydrocarbon analyses were performed on-site at a laboratory provided by SRL using automated headspace gas chromatography. These methods were implemented using a Hewlett-Packard 5890A Gas Chromatograph equipped with an HP 19395A Headspace Sampler, an HP3392A Networking Inegrator, an electron capture detector (ECD) for the halocarbons, and a flame ionization detector (FID) for the hydrocarbons. One halocarbon, vinyl chloride, was determined using the FID. For both halocarbons and hydrocarbons, a Supelco vocol wide bore capillary column, $60 \mathrm{~m} \times 0.75 \mathrm{~mm}$ I.D. with a $1.5 \mathrm{um} \mathrm{film}$, was used.

Analytical Standards. Calibration of the chromatograph for the light hydrocarbons, $\mathrm{C}_{1}-\mathrm{C}_{4}$, is achieved through the use of a commercial certified standard gas which is in the order of $10 \mathrm{ppm}$ for methane and $1 \mathrm{ppm}$ for the other hydrocarbons.

For the gasoline range hydrocarbons and the halocarbons, a standard solution is prepared composed of a blend of each compound. For the halocarbons (except vinyl chloride), the solvent used is methyl alcohol. For the gasoline range hydrocarbons, and vinyl chloride, the solvent is carbon disulfide. In both cases, the solution is made such that when $1 \mathrm{u} 1$ of standard solution is added to $8 \mathrm{gm}$ of soil in a headspace vial, 
the concentration of compound in the vial was increased as if the soil contained an additional $10 \mathrm{ppb}$ of compound. Thus, by adding appropriate volumes of standard solution to a headspace vial, several standardization points were achieved to form a calibration curve for each compound. A 100 to 1 dilution of the standard solution was used for standards less than $10 \mathrm{ppb}$. 
Detection Limits

Minimum detection limits for the light hydrocarbons, $\mathrm{C}_{1}-\mathrm{C}_{4}$, based on noise current when only pure nitrogen is injected is less than $1 \mathrm{ppb}$. In practice, using hand injection techniques, a $100 \mathrm{ppb}$ sample (standard) is reproducible $\pm 5 \mathrm{ppb}$.

For the headspace analysis of gasoline range hydrocarbons and halocarbons, reagent blanks were prepared by placing only the $\mathrm{Na}_{2} \mathrm{SO}_{4}-\mathrm{H}_{3} \mathrm{PO}_{4}$ solution in the headspace vials. At least one blank was prepared and analysed for every ten samples.

For the halocarbons, peak areas for compounds detected in 22 blanks were used to establish minimum values for detection limits which were set at the mean plus two standard deviations of the measured levels in the blanks.

For the gasoline-range hydrocarbons, only toluene could be detected in the blanks and the minimum detection level was set at the mean plus two standard deviations determined from 22 blank runs. For the remainder of the gasoline range hydrocarbons and vinyl chloride, the minimum detection level was estimated from a series of standard runs. In each case a standard equivalent to the presence of $10 \mathrm{ppb}$ or less (see Figures 50 and 51) was prepared and run. The minimum detection level quoted was estimated from the response to this standard.

Minimum detection levels for the halocarbons and gasoline range hydrocarbons are listed in Table 1. 
TABLE 1

MINIMUM DETECTION LEVELS (ppb)

\begin{tabular}{ll} 
Compound & MDL \\
pentane & 1.5 \\
hexane & 1.5 \\
heptane & 1.5 \\
octane & 1.5 \\
benzene & 1.5 \\
toluene & 2.0 \\
vinyl chloride & 3.0 \\
1,1 dichloroethylene & 0.3 \\
dichloromethane & 0.5 \\
trans l,2-dichloroethylene & 2.8 \\
cis 1,2-dichloroethylene & 4.0 \\
1,1-dichloroethane & 5.0 \\
1,2-dichloroethane & 5.0 \\
trichloromethane (chloroform) & 0.03 \\
trichloroethylene & 0.04 \\
tetrachloroethylene & 0.03 \\
\hline &
\end{tabular}




\section{Statistics}

Rudimentary statistical analyses were applied to the data for the purpose of deriving map symbols to illustrate the distribution of the species of interest.

For the light hydrocarbons, a mean $(m)$ and standard deviation $(\sigma)$ were determined for the magnitude of each hydrocarbon of interest. The map symbols relative to this distribution are as follows:

$$
\begin{aligned}
\boldsymbol{H} & =>m+2 \sigma \\
& =m+1.5 \sigma+m+2 \sigma \\
& =m+1.0 \sigma \rightarrow m+1.5 \sigma \\
0 & =m+m+1 \sigma \\
& =<m
\end{aligned}
$$

All samples in the 01d Ellenton Area were considered as one data set for statistical analyses. The $0 i 1$ Test Site and the Fire Training Area were considered separately.

For the halocarbons, since many of the sites were below the minimum detection levels, normal statistical parameters such as means and standard deviations could not be used to establish map parameters. Therefore, we have assumed that the data (or their logs) are normally distributed and have used conventional percentile distributions as map parameters. For example, the normal distribution is such that $2.28 \%$ of the sites (parameters) are more than $2 \sigma$ above the mean, $4.4 \%$ of the sites between $1.5 \sigma$ and $2.0 \sigma$ above the mean, etc. 


\section{Quality Assurance}

For the soil gas samples, field quality assurance included purging the soil gas probe at least five times between each site. Additionally, an air sample was taken every 10 sites (in addition to the first and last site of the day) and analyzed for long term contamination. Then by taking and exhausting a volume of air equal to the volume of the soil gas probe at each site before filling of the sample bottle, it was assured that only soil gas from the sampling depth was sampled.

For the soil samples, the sampling tube was carefully cleaned between each site. Then by collecting and discarding the first $18^{\prime \prime}$ of soil, the tube was abraded by a portion of the new sample.

Laboratory quality assurance was discussed above in Section II, Collection and Analysis of Samples. 
IV. Presentation and Discussion of Data

A. Old Ellenton: Area

The purpose of the investigations in the 01d Ellenton area shown in Figure 1 was to determine the presence or absence of contamination residual in the soils at several specified locations of highest probability of occurrence. Of particular interest were the 1ight hydrocarbons methane and butarie; the gasoline range hydrocarbons, pentane, hexane, heptane, octane, benzene and toluene; and the halocarbons, mainly tetrachoroethylene.

Methane is produced by anaerobic bacterial processes and could be indicative of abandoned septic tanks, buried decomposing organic material (trash) or other such sources.

Butane is the lowest molecular weight component of gasoline and the presence of anomalous butane with respect to methane, ethane and propane could be suggestive of gasoline spills or leaking gasoline tanks. Since the light hydrocarbons are relatively quickly determined analytically, they are an ideal survey tool for the presence of gasoline range hydrocarbons in water or soils using the relative quantity of butane as an indicator.

Soil analysis for the gasoline range hydrocarbons, a much more time consuming and expensive analytical procedure, was reserved for areas where evidence from the butane analysis (or other evidence) suggested the potential presence of these compounds. 
Soil analysis for halocarbons in the 01d Ellenton area was limited to the vicinity of the former dry cleaning store where potentially this material was used as a cleaning fluid.

As indicated on the 0ld Ellenton Area Index Map (Figure 1) a total of seven specific areas were surveyed and they will be discussed separately. 
FIGURE 1
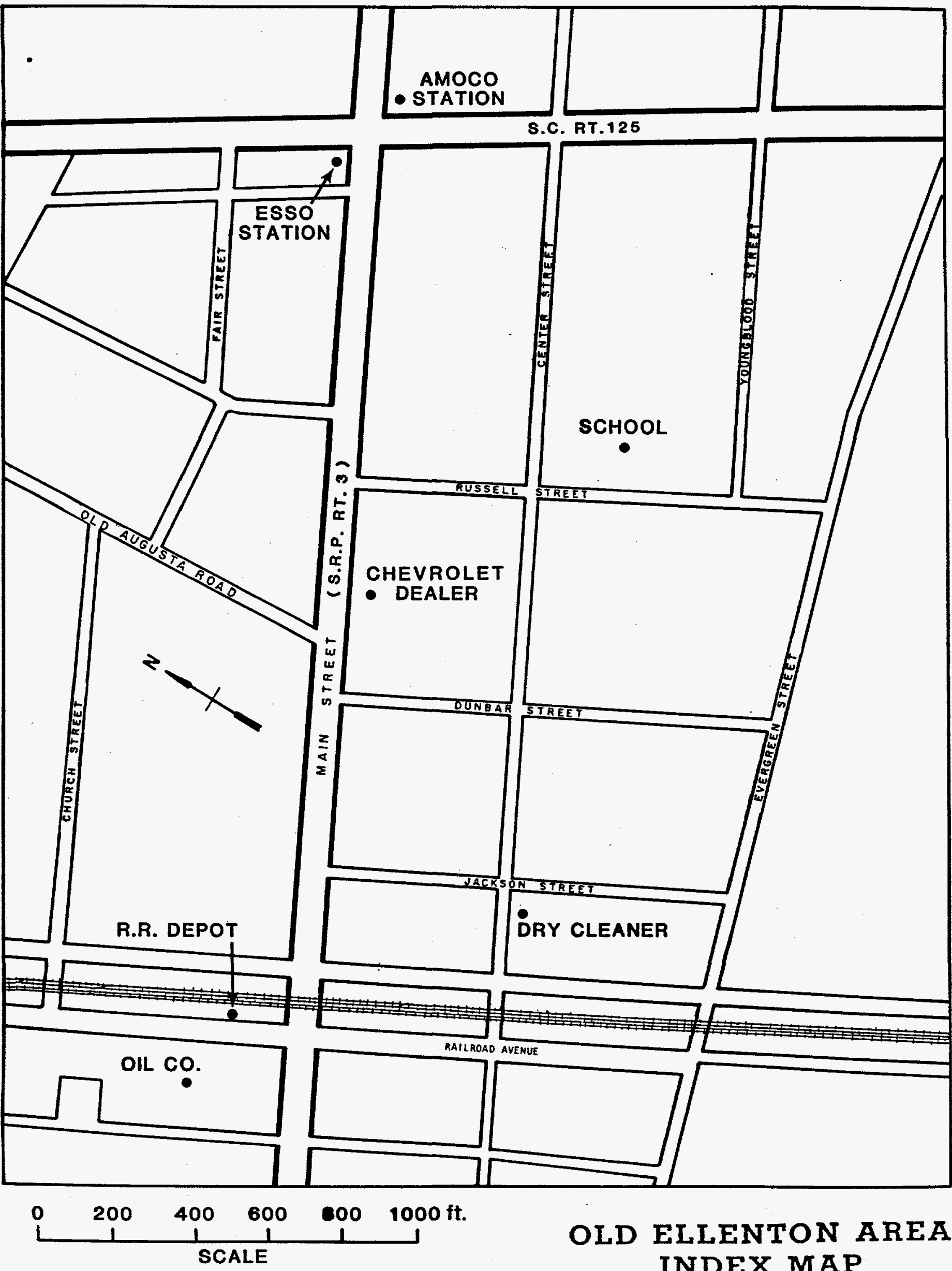

OLD ELLENTON AREA
INDEX MAP 


\section{Amoco Gasoline Station}

The 01d Ellenton Amoco gasoline station site is located on the eastern corner of the intersection of South Caroline Rt. 125 and Main Street (SRP Rt. 3), as shown on the Index Map (Figure 1). The building was vacated in the early to mid-1950's, at which time all surface structures were removed and the site graded over. Currently the site is relatively level, smooth and grass covered, with little or no surficial evidence which would indicate the existence of the original building foundation. Near surface soils are well drained and are composed predominantly of medium to fine grained sand with some silt, but little to no clay.

It was not known whether the underground gasoline tanks were removed at the time the gasoline station was demolished. We were provided with SRP photographs of the building before demolition and these enabled us to establish the approximate location of the building on the lot and the probable location of the underground storage tanks.

Based on the information described above, we initiated our sampling at the Amoco station, using a $7 \times 7$ site grid on 20 foot centers, both on and off the presumed location of the filling station as shown on the site location map on Figure 2. From this initial grid, a total of 46 soil gas samples were taken at a depth of $3 \mathrm{ft}$ and analyzed for $\mathrm{C}_{1}-\mathrm{C}_{4}$ hydrocarbons. The results of these analyses revealed anomalously high iso and normal butane (\% butane $=25.6$, see Table 3 ). This site was offset $1 \mathrm{ft}$ (site 13-R) and 
resampled to confirm the indication of anomalous butane at site 13 . At site $13-R$, butane was significantly higher $(\%$ butane $=53.5$, see Table 3) than at site 13, thus confirming the previous results.

Based on these results, we sampled 23 additional sites (sites 47-69) on 5 to $10 \mathrm{ft}$ centers in proximity to sites 13 and $13-\mathrm{R}$ as shown on Figure 2. In additions the sample probe was modified in order to obtain samples from $6 \mathrm{ft}$ depths at 14 sites. All of the data is recorded in Tables 2 and 3, and maps of pertinent parameters are shown in Figures 3, 4 and 5.

It is noted from Figures 4 and 5 that anomalous butane magnitudes and \% butanes are localized near the street intersection. During the sampling operation, a metallic object was encountered at several spots near site 52 at a depth of about 18 inches. The audible sound of the probe encountering the object led us to suspect it was a buried gasoline storage tank.

Consideration of the data from sites where both $3 \mathrm{ft}$ and $6 \mathrm{ft}$ samples were taken revealed a decrease of butane with depth at 13 of the 14 such sites. The one site which is excepted was a background (non-anomalous) site and the slight increase with depth at this site is considered normal. We conclude that the source of butane is near the surface, not at depth. This could result from spillage of gasoline during filling of the storage tank, a tank vent near the surface or overflows of automobile gasoline tanks. 
Based on the results of the light hydrocarbon analyses, soil samples were collected at 30 sites in the area of anomalous butanes. Soil samples were collected at a depth of 18 to 24 inches at the same sites used for soil gas samples. Soil samples are indicated on the Site Location Map on Figure 2.

As shown on Table 4, the only gasoline range hydrocarbons detected were $3.5 \mathrm{ppb}$ benzene and $2.0 \mathrm{ppb}$ toluene at Site 52 . No map of these data was made. The chromatographic trace of the sample at Site 52 is included as Figure 6.

Although some of the highest methane sites of this data set, shown on Figure 3, are found in the area of anomalous butanes, the highest site and several other high sites are not in this area. It is noticed that these latter sites are alligned near and parallel to the streets. On the other hand, the highest methane site is only about $6.8 \mathrm{ppm}$. This would not be considered to be of great concern. We have observed many methane levels in the 100 to $1000 \mathrm{ppm}$ range in otherwise pristine areas.

In conclusion, a localised area at the Amoco gasoline station in 01d Ellenton is characterized by anomalous levels of butane and anomalous $\%$ butanes (as measured relative to $C_{1}-C_{4}$ ). This area is probably that of buried gasoline storage tanks used in this business. Underground objects considered to be these tanks were encountered at about 18 inches depth, as discussed. Only trace residual gasoline range hydrocarbons, specifically benzene and toluene, were encountered. Based on these data, we conclude that the materials encountered are mostly due to surface spillage as opposed to tank leakage. 


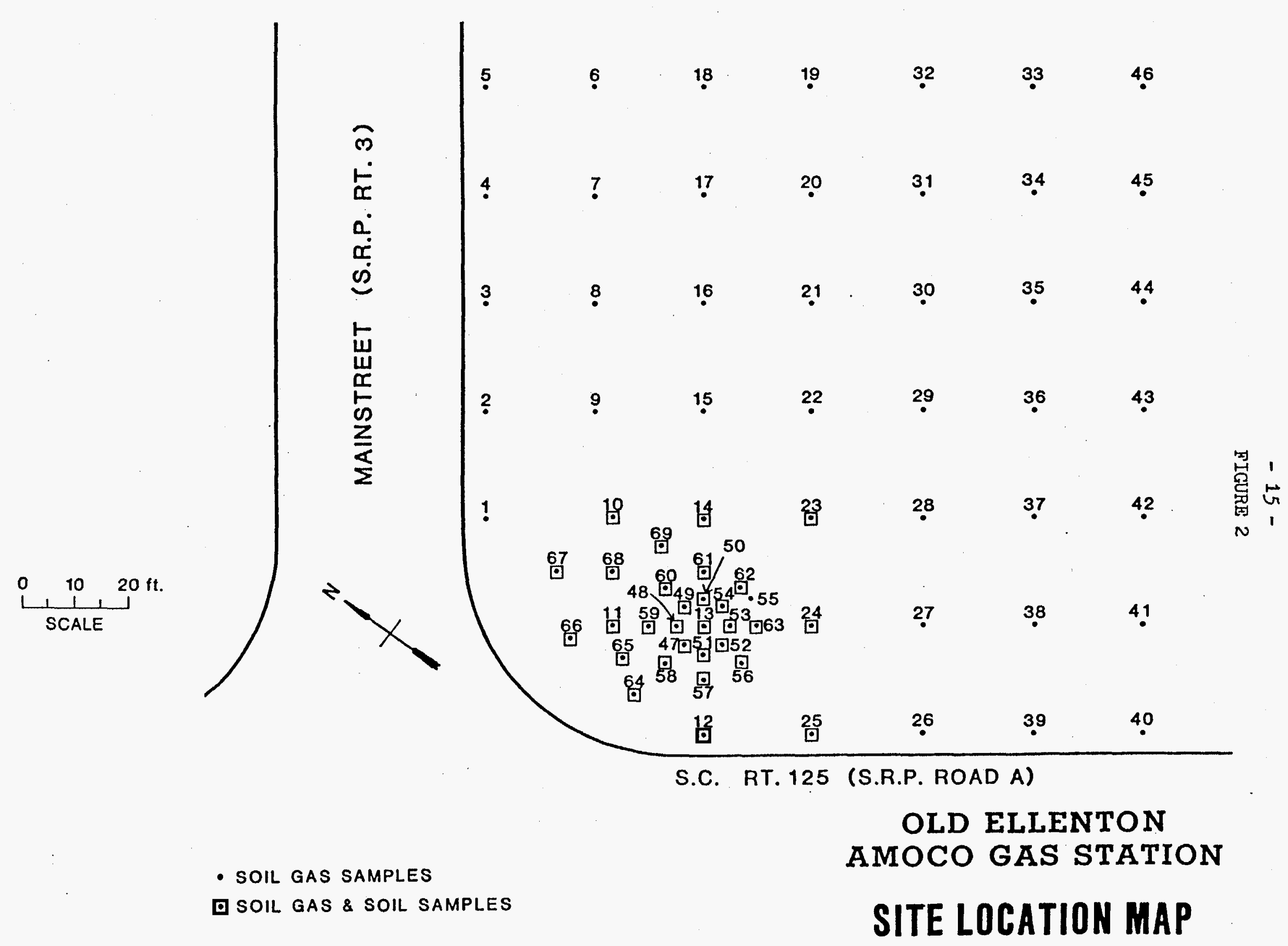

S.R.P.

NOVEMBER, 1986 



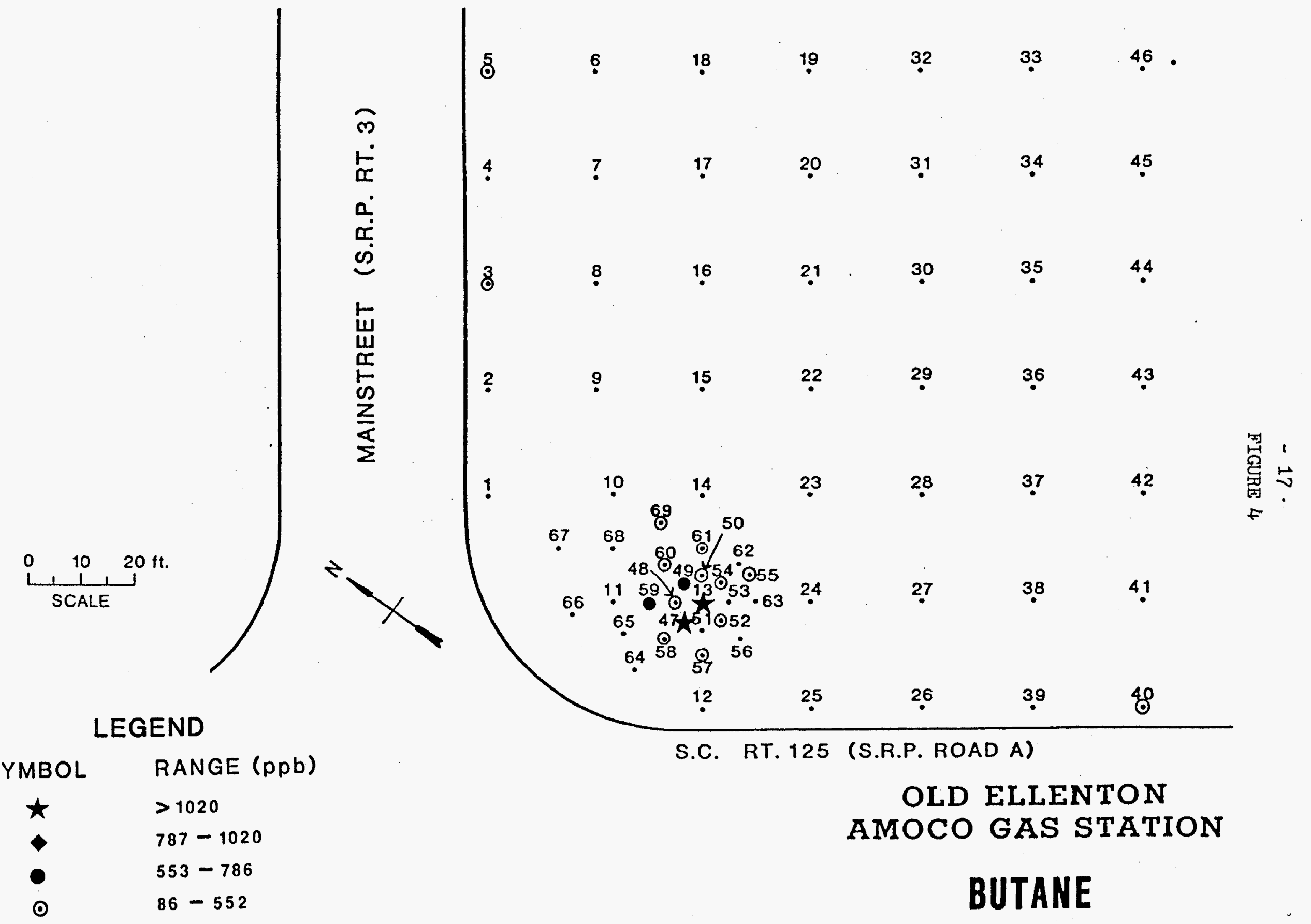

S.R.P.

NOVEMBER, 1986 


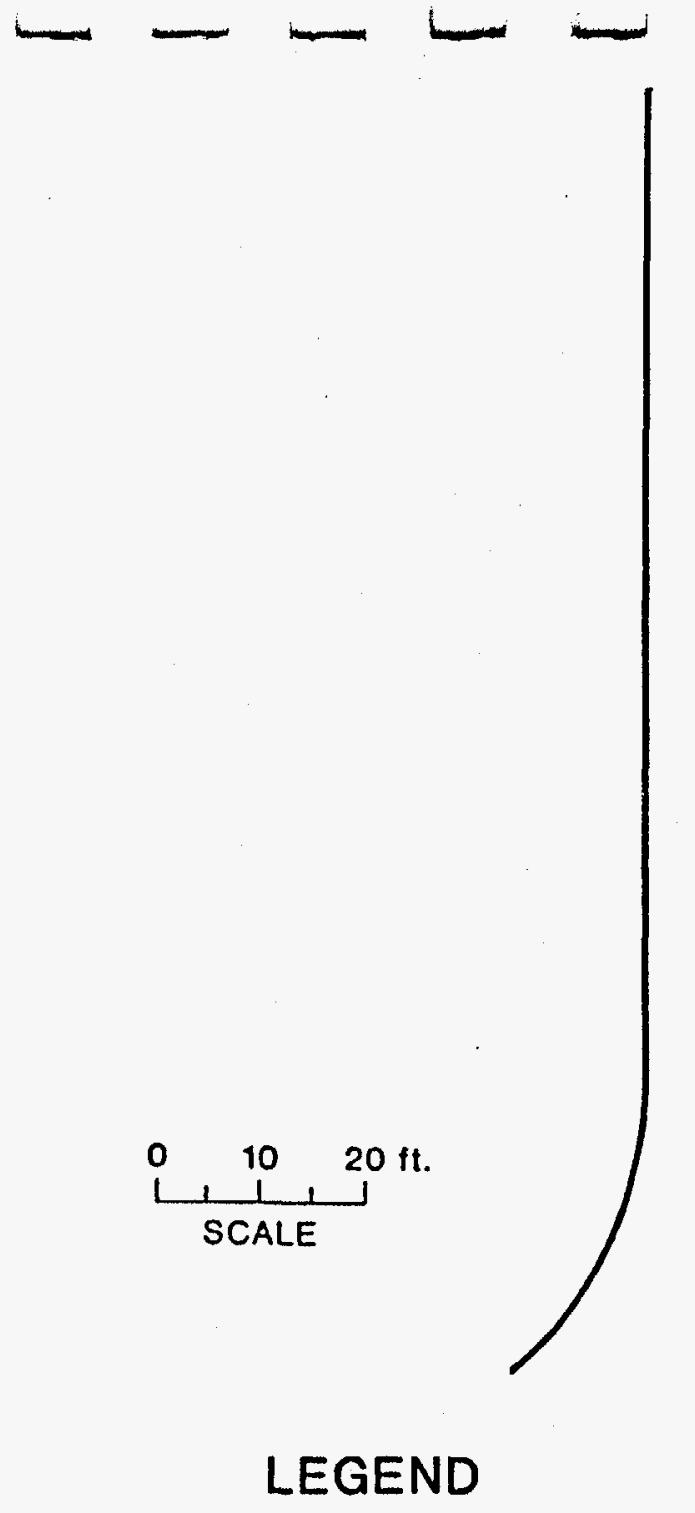

SYMBOL RANGE (\%)

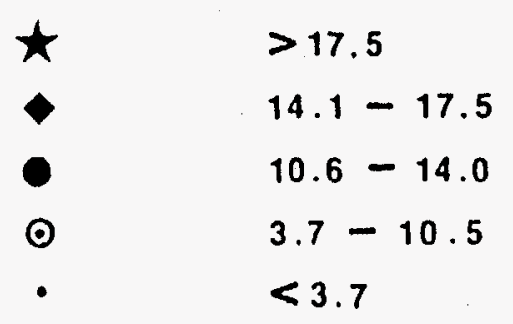

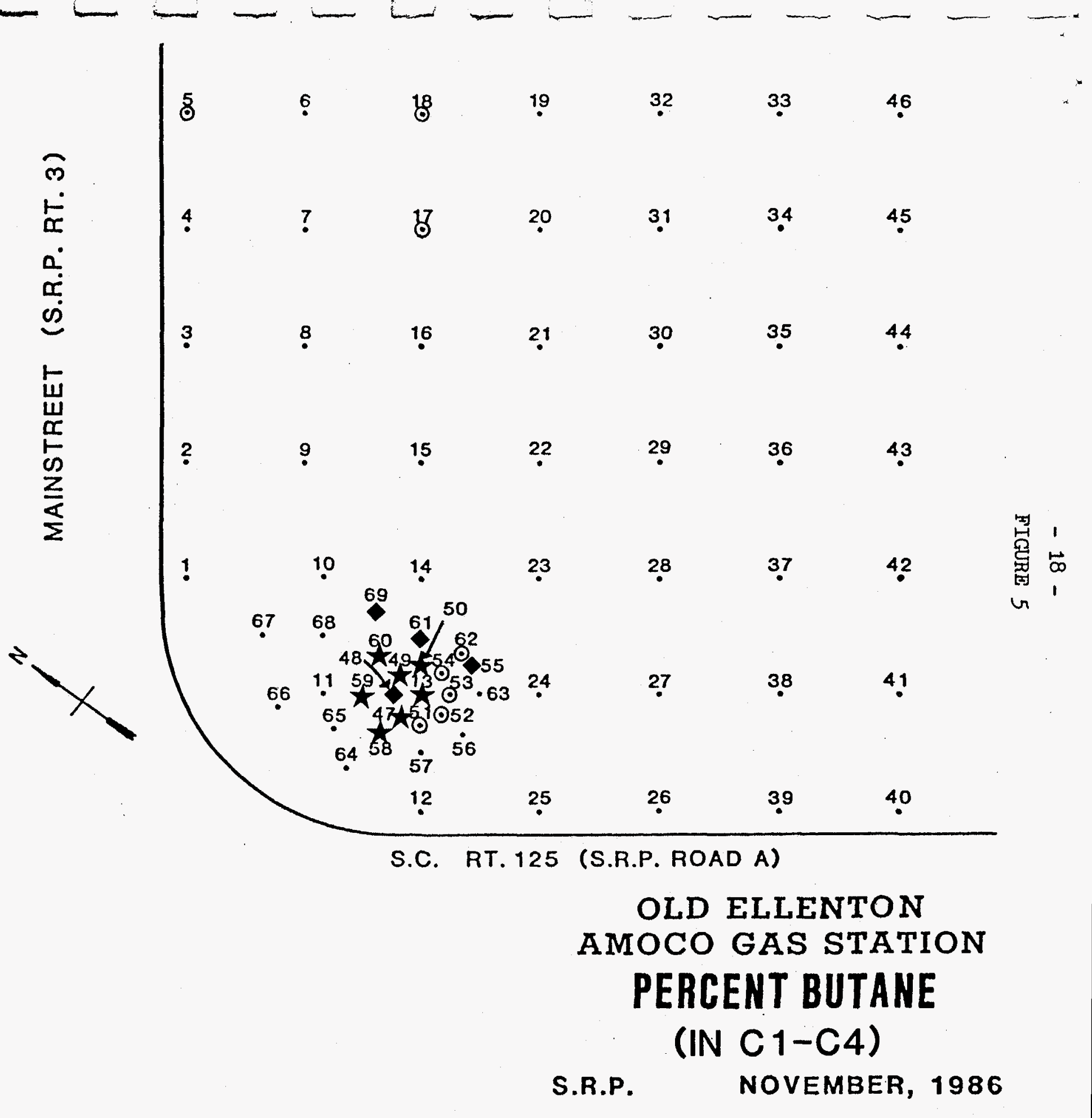


$A M-52$

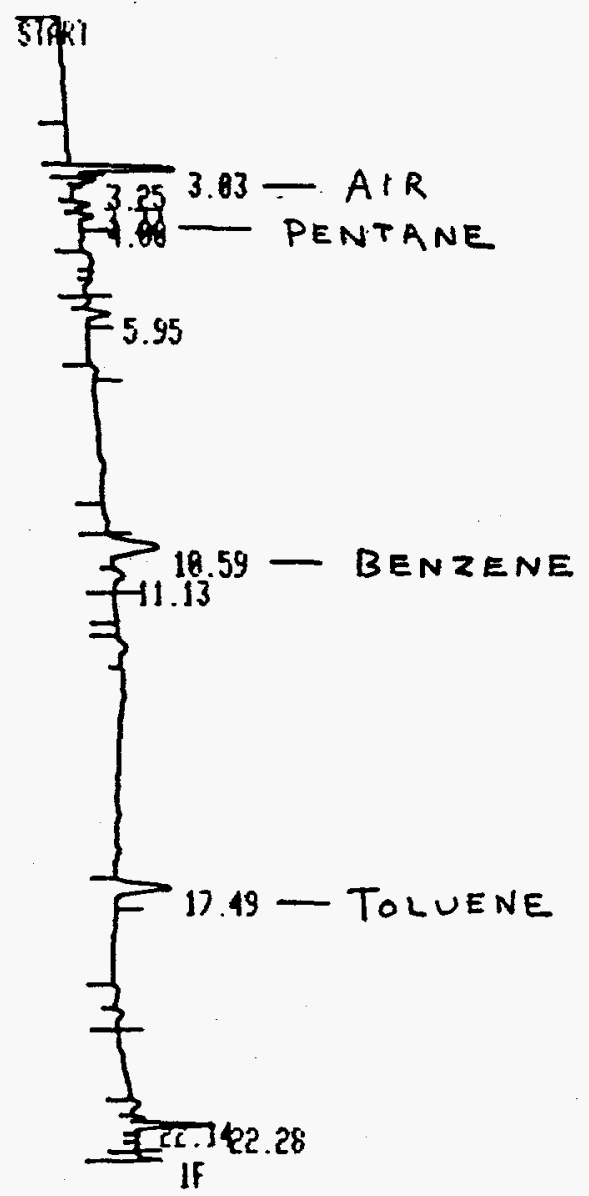

]

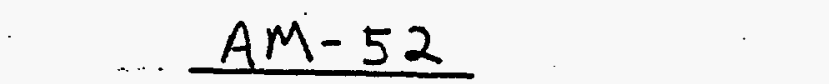

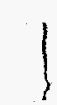
HORKF ILE MAME:

J

\begin{tabular}{|c|c|c|c|c|c|}
\hline PENTANE & $\begin{array}{c}\text { AREA\% } \\
\text { RT } \\
3.63 \\
3.25 \\
3.77 \\
4.98 \\
\end{array}$ & $\begin{array}{l}\text { AREA } \\
7822 \\
1222 \\
1193 \\
1533 \\
\end{array}$ & $\begin{array}{r}\text { TYPE } \\
\text { PE } \\
\text { PV } \\
\text { PV } \\
\text { YB } \\
\end{array}$ & $\begin{array}{l}A F / H T \\
\theta .102 \\
\theta .092 \\
0.183 \\
0.127 \\
\end{array}$ & $\begin{array}{r}\text { AREA\% } \\
19.394 \\
3.374 \\
3.294 \\
4.233 \\
\end{array}$ \\
\hline BENZENE & $\begin{array}{r}5.95 \\
10.59\end{array}$ & $\begin{array}{l}2279 \\
8819\end{array}$ & $\begin{array}{l}\text { PB } \\
\text { PY }\end{array}$ & $\begin{array}{l}0.136 \\
0.251 \\
\end{array}$ & $\begin{array}{r}6.268 \\
24.352 \\
\end{array}$ \\
\hline TOLUENE & $\begin{array}{l}11.13 \\
17.49\end{array}$ & $\begin{array}{r}1995 \\
6475 \\
\end{array}$ & $\begin{array}{l}\text { YB } \\
B B\end{array}$ & $\begin{array}{l}0.246 \\
8.173 \\
\end{array}$ & $\begin{array}{r}5.569 \\
17.879 \\
\end{array}$ \\
\hline & $\begin{array}{l}22.14 \\
22.28\end{array}$ & $\begin{array}{r}789 \\
4977\end{array}$ & $\begin{array}{l}P Y \\
Y Y\end{array}$ & $\begin{array}{l}0.082 \\
0.098\end{array}$ & $\begin{array}{r}1.958 \\
13.743\end{array}$ \\
\hline
\end{tabular}


TABLE 2

--- E. I. DUPONT DE MEMOURS CO. INC., S.R.P., ALKEN, S.C. ---

OLD ELLENTON / AMOCO STATION - PROBE SURVEY, SEPT, 1986 --.-..- SOIL GAS CONCENTRATIONS -..-

\begin{tabular}{|c|c|c|c|c|c|c|c|c|c|c|}
\hline \multicolumn{2}{|c|}{ SAMPLE } & $\begin{array}{r}\text { METHAHE } \\
\text { PPB }\end{array}$ & $\begin{array}{r}\text { ETHANE } \\
\text { PPB }\end{array}$ & $\begin{array}{c}\text { PROPAME I } \\
\text { PPB }\end{array}$ & $\begin{array}{l}\text {-BUTANE } \\
\text { PPB }\end{array}$ & $\begin{array}{c}\text { H-BUTANE E } \\
\text { PPB }\end{array}$ & $\begin{array}{c}\text { ETHYLNE } \\
\text { PPB }\end{array}$ & $\begin{array}{c}\text { PROPYLENE } \\
\text { PPB }\end{array}$ & \multicolumn{2}{|c|}{ SAMPLE } \\
\hline An & 1 & 739 & 38 & 16 & - & - & 41 & 12 & AH & 1 \\
\hline AH & 2 & 768 & 40 & 17 & - & - & 40 & 37 & All & 2 \\
\hline AH & 3 & 3841 & 201 & 69 & 72 & 19 & 126 & 140 & AH & 3 \\
\hline AH & 4 & 2014 & 99 & 34 & 56 & - & 74 & 81 & An & 4 \\
\hline AM & 5 & 6783 & 387 & 141 & 356 & 81 & 316 & 287 & AK & 5 \\
\hline AM & 6 & 711 & 47 & 22 & 22 & - & 39 & 41 & All & 6 \\
\hline Ah & 7 & 1232 & 19 & 16 & 19 & - & 29 & 24 & AM & 7 \\
\hline Ah & 8 & 1913 & 84 & 28 & 29 & - & 19 & 44 & AM & 8 \\
\hline AH & 9 & 1275 & 80 & 27 & 39 & - & 50 & 54 & AM & 9 \\
\hline AH & 10 & 849 & 45 & 19 & 17 & - & 44 & 46 & 解 & 10 \\
\hline All & 11 & 1623 & 80 & 26 & 39 & - & 48 & 45 & AH & 11 \\
\hline AK & 12 & 1478 & 66 & 25 & 25 & - & 48 & 46 & AH & 12 \\
\hline AK & 13 & 1420 & 58 & 39 & 411 & 111 & 31 & 29 & $A H$ & 13 \\
\hline AK & $13-R$ & 2594 & 301 & 3553 & 4738 & 2681 & 252 & 302 & AM & $13-R$ \\
\hline Al & 14 & 763 & 28 & 9 & 24 & - & 18 & 15 & An & 14 \\
\hline Al & 15 & 877 & 41 & 17 & 26 & - & 30 & 30 & AH & 15 \\
\hline An & 16 & 6.7 & 30 & 11 & 9 & - & 20 & 19 & AH & 16 \\
\hline An & 17 & 985 & 44 & 15 & 41 & - & 31 & 26 & Al & 17 \\
\hline AH & 18 & 1043 & 30 & 16 & 78 & - & 16 & 14 & Af & 18 \\
\hline Al & 19 & $6 \hat{7}$ & 15 & 8 & - & - & 12 & - & AlH & 19 \\
\hline AH & 20 & $8: 5$ & 31 & 11 & 11 & - & 19 & 15 & AN & 20 \\
\hline An & 21 & 799. & 19 & 7 & - & - & 13 & - & An & 21 \\
\hline An & 22 & 928 & 41 & 25 & 16 & - & 25 & 21 & All & 22 \\
\hline An & 23 & 847 & 42 & 15 & 21 & - & 28 & 25 & An & 23 \\
\hline AH & 24 & 1590 & 18 & 17 & 13 & - & 28 & 27 & AH & 24 \\
\hline An & 25 & 2870 & 118 & 39 & 58 & - & 73 & 67 & AH & 25 \\
\hline An & 26 & 2072 & 131 & 16 & 51 & - & 76 & 77 & An & 26 \\
\hline All & 27 & 934 & 47 & 18 & 22 & - & 29 & 31 & AH & 27 \\
\hline AH & 28 & 695 & 28 & 11 & - & - & 18 & 12 & AM & 28 \\
\hline All. & 29 & 488 & 18 & 0 & - & - & 14 & - & An & 29 \\
\hline AH & 30 & 1198 & 18 & - & - & - & 20 & - & AH & 30 \\
\hline A月 & 31 & 1000 & 23 & 9 & 37 & - & 19 & 13 & An & 31 \\
\hline All & 32 & 619 & 25 & 10 & 14 & - & 20 & 18 & AH & 32 \\
\hline An & 33 & 598 & 21 & 8 & - & - & 16 & 14 & AN & 33 \\
\hline A. & 34 & 891 & 31 & 12 & 28 & - & 17 & 17 & AH & 34 \\
\hline AM & 35 & 881 & 26 & 13 & - & - & 14 & 13 & An & 35 \\
\hline An & 36 & 442 & 18 & 6 & - & - & 18 & - & AM & 36 \\
\hline AH & 37 & 574 & 22 & 10 & - & - & 18 & 15 & AH & 37 \\
\hline All & 38 & 654 & 29 & 13 & 10 & - & 24 & 22 & An & 38 \\
\hline AN & 39 & 1536 & 81 & 27 & 37 & - & 49 & 46 & AH & 39 \\
\hline AM & 40 & 2638 & 123 & 17 & 72 & 24 & 104 & 84 & All & 40 \\
\hline AK & 41 & 2174 & 147 & 64 & 48 & 11 & 161 & 131 & AK & 41 \\
\hline AM & 42 & 913 & 41 & 25 & 22 & - & 12 & 30 & All & 12 \\
\hline AK & 43 & 1464 & 56 & 26 & 16 & - & 50 & 46 & AK & 43 \\
\hline AM & 44 & 820 & 26 & 10 & 11 & - & 17 & 12 & AH & 44 \\
\hline AH & 45 & 428 & 16 & 9 & - & - & 12 & - & AK & 45 \\
\hline AH & 46 & 770 & 26 & 10 & - & - & 19 & - & An & 16 \\
\hline AM & 47 & 1044 & 53 & 142 & 1184 & 178 & 33 & 36 & An & 17 \\
\hline AM & $47-R$ & 1491 & 84 & 61 & 562 & 50 & 18 & 48 & An & $17-R$ \\
\hline
\end{tabular}


TABLE 2 (cont)

--- E. 1. DUPONT DE NEKOURS CO. IKC., S.R.P., AIKEN, S.C. ---

-..- OLD ELLENTON / AHOCO STATION - PROBE SURVEY, SEPT. 1986 -..-.-- SOIL GAS CONCENTRATIONS -..--

\begin{tabular}{|c|c|c|c|c|c|c|c|c|c|c|}
\hline SAM: & & $\begin{array}{r}\text { METHANE } \\
\text { PPB }\end{array}$ & $\begin{array}{r}\text { ETHANE } \\
\text { PPB }\end{array}$ & $\begin{array}{r}\text { PROPANE } \\
\text { PPB }\end{array}$ & $\begin{array}{c}\text { 1-BUTANE } \\
\text { PPB }\end{array}$ & $\begin{array}{c}\text { N-BUTANE E } \\
\text { PPB }\end{array}$ & $\begin{array}{c}\text { ETHYLENE } \\
\text { PPB }\end{array}$ & $\begin{array}{c}\text { PROPYLENE } \\
\text { PPB }\end{array}$ & & PLE \\
\hline Ar & $47-6$ & $8: 5$ & 38 & 31 & 473 & 31 & 31 & 32 & An & $47-6$ \\
\hline AH & 48 & 1130 & 45 & 32 & 194 & 23 & 31 & 28 & AM & 48 \\
\hline AM & $48-6$ & 907 & 29 & 11 & 53 & - & 18 & 21 & AK & $48-6$ \\
\hline AK & 49 & 1159 & 48 & 35 & 515 & 103 & 31 & 32 & AK & 49 \\
\hline Ah & $19-6$ & 1028 & 30 & 11 & 127 & - & 16 & 18 & AM & $49-6$ \\
\hline AK & 50 & 1035 & 39 & 28 & 216 & 39 & 33 & 29 & AM & 50 \\
\hline AII & $50-6$ & 951 & 15 & - & 17 & - & 8 & - & AN & $50-6$ \\
\hline AH & 51 & 1019 & 30 & 12 & 48 & - & 24 & 15 & AH & 51 \\
\hline All & $51-6$ & 725 & 31 & 13 & 21 & - & 21 & 26 & All & $51-6$ \\
\hline AL & 52 & 3912 & 448 & 247 & 162 & 167 & 480 & 570 & AK & 52 \\
\hline AH & $52-6$ & 984 & 27 & 11 & 33 & - & 19 & 24 & AH & $52-6$ \\
\hline AH & 53 & 1043 & 38 & 16 & 54 & - & 27 & 27 & An & 53 \\
\hline AH & 54 & 1623 & 98 & 61 & 81 & 48 & 76 & 64 & AH & 54 \\
\hline AH & $54-t$ & 977 & 29 & 10 & 24 & - & 20 & 25 & AH & $54-6$ \\
\hline AM & 55 & 1090 & 36 & 16 & 212 & 19 & 38 & 28 & AH & 55 \\
\hline All & $55-t$ & 38639 & 35 & 18 & 22 & - & 22 & 35 & AM & $55-6$ \\
\hline AM & 56 & 1130 & 27 & 10 & 19 & - & 20 & 15 & AK & 56 \\
\hline AH & $56-i$ & 1101 & 38 & 14 & 29 & - & 21 & 24 & AK & $56-6$ \\
\hline AK & 57 & 2435 & 179 & 70 & 57 & 35 & 174 & 149 & AH & 57 \\
\hline AH & $57-\dot{0}$ & 927 & 35 & 13 & 25 & - & 24 & 32 & Al & $57-6$ \\
\hline AH & 58 & 1217 & 71 & 188 & 223 & 113 & 70 & 52 & AM & 58 \\
\hline AH & $58-5$ & 714 & 22 & 9 & 19 & - & 16 & 20 & All & $58-6$ \\
\hline AH & 59 & 980 & 43 & 29 & 599 & 65 & 27 & 22 & AH & 59 \\
\hline AK & $59-6$ & 1047 & 30 & 11 & 40 & - & 20 & 22 & All & $59-6$ \\
\hline An & 60 & 896 & 37 & 19 & 262 & - & 25 & 26 & AN & 60 \\
\hline AH & $60-6$ & 990 & 15 & 8 & 30 & - & 10 & 13 & AH & $60-6$ \\
\hline AN & 61 & 1159 & 64 & 103 & 189 & 66 & 44 & 35 & All & 61 \\
\hline Al & $61-6$ & 1143 & 23 & 8 & 21 & - & 11 & - & An & $61-6$ \\
\hline AH & 62 & 1232 & 46 & 21 & 55 & - & 40 & 32 & An & 62 \\
\hline AH & 63 & 1348 & 38 & 17 & 23 & - & 37 & 28 & Al & 63 \\
\hline AH & 64 & 1477 & 62 & 23 & 54 & - & 46 & 49 & AN & 64 \\
\hline AY & 65 & 831 & 25 & 9 & 16 & - & 18 & 18 & AH & 65 \\
\hline An & 66 & 965 & 12 & - & - & - & 12 & - & All & 66 \\
\hline AH & 67 & 1156 & 47 & 19 & 33 & - & 40 & 39 & AH & 67 \\
\hline An & 68 & 872 & 34 & 12 & 25 & - & 21 & 22 & AK & 68 \\
\hline AK & 69 & 761 & 11 & 15 & 142 & - & 23 & 30 & AK & 69 \\
\hline
\end{tabular}

NOTE:

$$
\begin{aligned}
& \text { AH } X X-3 \text { FT. SAMPLE } \\
& \text { AH } X X-6-6 \text { FT. SAMPLE } \\
& \text { AH } X X-R \text { - REPEAT SAMPLE }
\end{aligned}
$$


TABLE 3

--- E. 1. DUPONT DE MEMOURS CO. INC., S.R.P., AIKEN, S.C. ---

--- DLD ELLENTON / AMOCO STATION - PROBE SURVEY, SEPT. 1986 -..-

---- SOIL GAS PERCENTAGES ----

\begin{tabular}{|c|c|c|c|c|c|c|c|c|c|c|c|}
\hline \multicolumn{2}{|c|}{ SAMPLE } & $\begin{array}{l}\text { TOTAL } \\
\text { Cl-C4 }\end{array}$ & $\begin{array}{r}\text { METHANE } \\
\text { ZCI }\end{array}$ & $\begin{array}{r}\text { ETHANE } \\
\text { IC2 }\end{array}$ & $\begin{array}{l}\text { PROPANE } \\
\text { ICJ }\end{array}$ & $\begin{array}{r}\text { BUTANE } \\
\text { 2CA }\end{array}$ & $\begin{array}{c}\text { TOTAL } \\
\text { HYDCARB }\end{array}$ & $\begin{array}{c}\text { ETHYLENE } \\
z\end{array}$ & $\begin{array}{c}\text { YLERE } \\
?\end{array}$ & \multicolumn{2}{|c|}{ SAMPLE } \\
\hline AH & 1 & 793 & 93.2 & 4.8 & 2.0 & 0.0 & 876 & 4.7 & 4.8 & An & 1 \\
\hline Al & 2 & 825 & 93.1 & 4.8 & 2.1 & 0.0 & 902 & 4.4 & 4.1 & AH & 2 \\
\hline AM & 3 & 4202 & 91.4 & 4.8 & 1.6 & 2.2 & 4468 & 2.8 & 3.1 & AK & 3 \\
\hline$A 5$ & 1 & 2203 & 91.4 & 4.5 & 1.5 & 2.5 & 2358 & 3.1 & 3.4 & AK & 4 \\
\hline AH & 5 & 7748 & 87.5 & 5.0 & 1.8 & 5.6 & 8351 & 3.8 & 3.4 & AK & 5 \\
\hline AH & 6 & 802 & 88.7 & 5.9 & 2.7 & 2.7 & 882 & 4.1 & 4.6 & An & 6 \\
\hline A月 & 7 & 1316 & 93.6 & 3.7 & 1.2 & 1.4 & 1369 & 2.1 & 1.8 & AN & 7 \\
\hline AH & 8 & 2054 & 93.1 & 4.1 & 1.4 & 1.4 & 2147 & 2.3 & 2.0 & AN & 8 \\
\hline Aln & 9 & 1421 & 89.7 & 5.6 & 1.9 & 2.7 & 1525 & 3.3 & 3.5 & AN & 9 \\
\hline An & 10 & 930 & 91.3 & 4.8 & 2.0 & 1.8 & 1020 & 1.3 & 4.5 & An & 10 \\
\hline Al & 11 & 1768 & 91.8 & 4.5 & 1.5 & 2.2 & $186 !$ & 2.6 & 2.4 & An & 11 \\
\hline AH & 12 & 1594 & 92.7 & 4.1 & 1.6 & 1.6 & 1688 & 2.8 & 2.7 & All & 12 \\
\hline An & 13 & 2039 & 69.6 & 2.8 & 1.9 & 25.6 & 2099 & 1.5 & 1.4 & All & 13 \\
\hline AH. & $13-R$ & 13867 & 18.7 & 2.2 & 25.6 & 53.5 & 14421 & 1.7 & 2.1 & An & $13-R$ \\
\hline An & 14 & 829 & 92.6 & 3.4 & 1.1 & 2.9 & 862 & 2.1 & 1.7 & AM & 14 \\
\hline AI & 15 & 961 & 91.3 & 4.3 & 1.8 & 2.7 & 1021 & 2.9 & 2.9 & AH & 15 \\
\hline All & 16 & 707 & 92.9 & 4.2 & 1.6 & 1.3 & 746 & 2.7 & 2.5 & AK & 16 \\
\hline All & 17 & 1085 & 90.8 & 4.1 & 1.4 & 3.8 & 1142 & 2.7 & 2.3 & AK & 17 \\
\hline Al & 18 & 1167 & 89.4 & 2.6 & 1.4 & 6.7 & 1197 & 1.3 & 1.2 & AK & 18 \\
\hline An & 19 & 632 & 96.4 & 2.4 & 1.3 & 0.0 & 644 & 1.9 & 0.0 & AK & 19 \\
\hline Al & 20 & 928 & 94.3 & 3.3 & 1.2 & 1.2 & 962 & 2.0 & 1.6 & AN & 20 \\
\hline Af & 21 & 825 & 96.8 & 2.3 & 0.8 & 0.0 & 838 & 1.6 & 0.0 & AN & 21 \\
\hline AH & 22 & 1010 & 91.9 & 1.1 & 2.5 & 1.6 & 1056 & 2.4 & 2.0 & AK & 22 \\
\hline AH & 23 & 925 & 91.6 & 4.5 & 1.6 & 2.3 & 978 & 2.9 & 2.6 & AK & 23 \\
\hline AH & 24 & 1658 & 95.3 & 2.9 & 1.0 & 0.8 & 1713 & 1.6 & 1.6 & AK & 24 \\
\hline AH & 25 & 3085 & 93.0 & 3.8 & 1.3 & 1.9 & 3225 & 2.3 & 2.1 & AM & 25 \\
\hline AM & 26 & 2300 & 90.1 & 5.7 & 2.0 & 2.2 & 2453 & 3.1 & 3.1 & AK & 26 \\
\hline AH & 27 & 1021 & 91.5 & 4.6 & 1.8 & 2.2 & 1081 & 2.7 & 2.9 & All & 27 \\
\hline AK & 28 & 734 & 94.7 & 3.8 & 1.5 & 0.0 & 764 & 2.4 & 1.6 & AK & 28 \\
\hline AK & 29 & 506 & 96.1 & 3.6 & 0.0 & 0.0 & 520 & 2.7 & 0.0 & AM & 29 \\
\hline Ah & 30 & 1216 & 98.5 & 1.5 & 0.0 & 0.0 & 1236 & 1.6 & 0.0 & AM & 30 \\
\hline AM & 31 & 1069 & 93.5 & 2.2 & 0.8 & 3.5 & 1101 & 1.7 & 1.2 & AM & 31 \\
\hline AH & 32 & 668 & 92.7 & 3.7 & 1.5 & 2.1 & 706 & 2.8 & 2.5 & AM & 32 \\
\hline AH & 33 & 627 & 95.4 & 3.3 & 1.3 & 0.0 & 657 & 2.1 & 2.1 & AM & 33 \\
\hline AH & 34 & 962 & 92.6 & 3.2 & 1.2 & 2.9 & 996 & 1.7 & 1.7 & An & 34 \\
\hline A月 & 35 & 920 & 95.8 & 2.8 & 1.4 & 0.0 & 947 & 1.5 & 1.1 & AM & 35 \\
\hline AY & 36 & 166 & 94.8 & 3.9 & 1.3 & 0.0 & 484 & 3.7 & 0.0 & AM & 36 \\
\hline Al & 37 & 606 & 94.7 & 3.6 & 1.7 & 0.0 & 639 & 2.8 & 2.3 & AH & 37 \\
\hline AH & 38 & 706 & 92.6 & 4.1 & 1.8 & 1.4 & 752 & 3.2 & 2.9 & AK & 38 \\
\hline AH & 39 & 1681 & 91.1 & 4.8 & 1.6 & 2.2 & 1776 & 2.8 & 2.6 & AK & 39 \\
\hline AH & 40 & 2904 & 90.8 & 4.2 & 1.6 & 3.3 & 3092 & 3.4 & 2.7 & An & 40 \\
\hline AK & 41 & 2444 & 89.0 & 6.0 & 2.6 & 2.4 & 2736 & 5.9 & 4.8 & AM & 11 \\
\hline AM & 42 & 1001 & 91.2 & 4.1 & 2.5 & 2.2 & 1073 & 3.9 & 2.8 & AM & 12 \\
\hline All & 43 & 1562 & 93.7 & 3.6 & 1.7 & 1.0 & 1658 & 3.0 & 2.8 & AH & 43 \\
\hline An & 44 & 867 & 94.6 & 3.0 & 1.2 & 1.3 & 896 & 1.9 & 1.3 & An & 44 \\
\hline AH & 45 & 453 & 94.5 & 3.5 & 2.0 & 0.0 & 465 & 2.6 & 0.0 & AK & 45 \\
\hline Al & 46 & 806 & 95.5 & 3.2 & 1.2 & 0.0 & 825 & 2.3 & 0.0 & An & 46 \\
\hline AH & 47 & 2601 & 40.1 & 2.0 & 5.5 & 52.4 & 2670 & 1.2 & 1.3 & An & 17 \\
\hline AM & $47-R$ & 2248 & 66.3 & 3.7 & 2.7 & 27.2 & 2344 & 2.0 & 2.0 & AM & $47-R$ \\
\hline
\end{tabular}


--- E. 1. DUPOKT DE MEMOURS CO. INC., S.R.P., AIKEN, S.C. ----

--- DLD ELLENTON / AMOCO STATION - PROBE SURUEY, SEPT. 1986 ----.-- SOIL GAS PERCENTAGES ----

\begin{tabular}{|c|c|c|c|c|c|c|c|c|c|c|c|}
\hline \multicolumn{2}{|c|}{$\begin{array}{c}\text { SAMPLE } \\
\vdots\end{array}$} & \multirow{2}{*}{$\begin{array}{l}\text { TOTAL } \\
\text { CI-CA } \\
1408\end{array}$} & \multirow{2}{*}{$\begin{array}{r}\text { METHANE } \\
\text { ZC1 } \\
59.3\end{array}$} & \multirow{2}{*}{$\begin{array}{r}\text { ETHANE } \\
\text { IC2 }\end{array}$} & \multirow{2}{*}{$\begin{array}{r}\text { PROPANE } \\
\text { YC3 } \\
2.2\end{array}$} & \multirow{2}{*}{$\begin{array}{r}\text { BUTANE } \\
\text { 2C4 } \\
35.8\end{array}$} & \multirow{2}{*}{$\begin{array}{c}\begin{array}{c}\text { TOTAL } \\
\text { HYDCARB }\end{array} \\
1471\end{array}$} & \multicolumn{2}{|c|}{$\begin{array}{c}\text { ETHYLENE PROPYLENE } \\
2\end{array}$} & \multicolumn{2}{|c|}{ SAMPLE } \\
\hline AM & $47-6$ & & & & & & & 2.1 & 2.2 & All & $17-6$ \\
\hline AH & 48 & 1424 & 79.4 & 3.2 & 2.2 & 15.2 & 1483 & 2.1 & 1.9 & Af & 48 \\
\hline An & $48-6$ & 1000 & 90.7 & 2.9 & 1.1 & 5.3 & 1039 & 1.7 & 2.0 & AY & $48-6$ \\
\hline AH & 19 & 1860 & 62.3 & 2.6 & 1.9 & 33.2 & 1923 & 1.6 & 1.7 & An & 49 \\
\hline AH & $19-6$ & 1196 & 86.0 & 2.5 & 0.9 & 10.6 & 1230 & 1.3 & 1.5 & AK & $49-6$ \\
\hline AH & 50 & 1356 & 76.3 & 2.9 & 2.1 & 18.7 & 1418 & 2.3 & 2.0 & AN & 50 \\
\hline An & $50-6$ & 983 & 96.7 & 1.5 & 0.0 & 1.7 & 991 & 0.8 & 0.0 & AH & $50-6$ \\
\hline AM & 51 & 1109 & 91.9 & 2.7 & 1.1 & 4.3 & 1148 & 2.1 & 1.3 & AH & $5 !$ \\
\hline AH & $51-6$ & 790 & 91.8 & 3.9 & 1.6 & 2.7 & 837 & 2.5 & 3.1 & AH & $51-6$ \\
\hline An & 52 & 1966 & 79.4 & 9.0 & 5.0 & 6.6 & 6016 & 8.0 & 9.5 & AH & 52 \\
\hline AM & $57-6$ & 1055 & 93.3 & 2.6 & 1.0 & 3.1 & 1098 & 1.7 & 2.2 & All & $52-6$ \\
\hline AH & 5 & 1151 & 90.6 & 3.3 & 1.4 & 4.7 & 1205 & 2.2 & 2.2 & AH & 53 \\
\hline AH & $5:$ & 1911 & 81.9 & 5.1 & 3.2 & 6.8 & 2051 & 3.7 & 3.1 & An & 54 \\
\hline AK & $5--6$ & 1040 & 93.9 & 2.8 & 1.0 & 2.3 & 1085 & 1.8 & 2.3 & AK & $54-6$ \\
\hline AM & $5 \bar{J}$ & 1373 & 79.1 & 2.6 & 1.2 & 16.8 & 1439 & 2.6 & 1.9 & AK & 55 \\
\hline AH & $: 5-6$ & 38714 & 99.8 & 0.1 & 0.0 & 0.1 & 38771 & 0.1 & 0.1 & An & $55-6$ \\
\hline AK & 56 & 1186 & 95.3 & 2.3 & 0.8 & 1.6 & 1221 & 1.6 & 1.2 & An & 56 \\
\hline AH & $=6-6$ & 1182 & 93.1 & 3.2 & 1.2 & 2.5 & 1227 & 1.7 & 2.0 & An & $56-6$ \\
\hline An & 57 & 2776 & 87.7 & 6.4 & 2.5 & 3.3 & 3099 & 5.6 & 4.8 & An & 57 \\
\hline AK & $57-6$ & 1000 & 92.7 & 3.5 & 1.3 & 2.5 & 1056 & 2.3 & 3.0 & An & $57-6$ \\
\hline An & 58 & 1812 & 67.2 & 3.9 & 10.4 & 18.5 & 1934 & 3.6 & 2.7 & An & 58 \\
\hline Ah & $58-6$ & 764 & 93.5 & 2.9 & 1.2 & 2.5 & 800 & 2.0 & 2.5 & AK & $58-6$ \\
\hline AH & 59 & 1716 & 57.1 & 2.5 & 1.7 & 38.7 & 1765 & 1.5 & 1.2 & An & 59 \\
\hline An & $59-6$ & 1128 & 92.8 & 2.7 & 1.0 & 3.5 & 1170 & 1.7 & 1.9 & AK & $59-6$ \\
\hline AM & 60 & 1214 & 73.8 & 3.0 & 1.6 & 21.6 & 1265 & 2.0 & 2.1 & AK & 60 \\
\hline AY & $60-6$ & 1043 & 94.9 & 1.4 & 0.8 & 2.9 & 1066 & 0.9 & 1.2 & AK & $60-6$ \\
\hline An & 61 & 1581 & 73.3 & 4.0 & 6.5 & 16.1 & 1660 & 2.7 & 2.1 & AM & 61 \\
\hline$A K$ & $61-6$ & 1195 & 95.6 & 1.9 & 0.7 & 1.8 & 1206 & 0.9 & 0.0 & AY & $61-6$ \\
\hline AK & 62 & 1351 & 91.0 & 3.4 & 1.6 & 4.1 & 1426 & 2.8 & 2.2 & All & 62 \\
\hline Ah & 63 & 1426 & 94.5 & 2.7 & 1.2 & 1.6 & 1491 & 2.5 & 1.9 & AK & 63 \\
\hline AH & 64 & 1616 & 91.4 & 3.8 & 1.1 & 3.3 & 1711 & 2.7 & 2.9 & AK & 64 \\
\hline AH & 65 & 881 & 94.3 & 2.8 & 1.0 & 1.8 & 917 & 2.0 & 2.0 & AH & 65 \\
\hline AH & 66 & 977 & 98.8 & 1.2 & 0.0 & 0.0 & 989 & 1.2 & 0.0 & AK & 66 \\
\hline AM & $b ?$ & 1255 & 92.1 & 3.7 & 1.5 & 2.6 & 1334 & 3.0 & 2.9 & AH & 67 \\
\hline An & 68 & 913 & 92.5 & 3.6 & 1.3 & 2.7 & 986 & 2.1 & 2.2 & An & 68 \\
\hline AK & 69 & 959 & 79.4 & 4.3 & 1.6 & 14.8 & 1012 & 2.3 & 3.0 & AH & 69 \\
\hline
\end{tabular}

MOTE:

$$
\begin{aligned}
& \text { AH } X X-3 \text { FT. SAKPLE } \\
& \text { AH } X X-6-6 \text { FT. SAMPLE } \\
& \text { AH } X X-R-\text { REPEAT SAMPLE }
\end{aligned}
$$



-..- SOIL CONCENTRATIONS BY MEIGHT -..-

\begin{tabular}{|c|c|c|c|c|c|c|c|c|c|c|}
\hline $\begin{array}{l}\text { SAMPLE } \\
\text { MUMBER }\end{array}$ & DEPTH & $\begin{array}{r}\text { PENTANE } \\
\text { PPB }\end{array}$ & $\begin{array}{r}\text { HEXANE } \\
\text { PPB }\end{array}$ & $\begin{array}{r}\text { HEPTANE } \\
\text { PPB }\end{array}$ & $\begin{array}{r}\text { BENZENE } \\
\text { PPB }\end{array}$ & $\begin{array}{r}\text { BCTANE } \\
\text { PPB }\end{array}$ & $\begin{array}{r}\text { TOLUENE } \\
\text { PPB }\end{array}$ & $\begin{array}{r}\text { TOTAL } \\
\text { AREA }\end{array}$ & $\begin{array}{l}\text { SAMFLE } \\
\text { NUMBER }\end{array}$ & DEPTH \\
\hline An 10 & 2.0 & - & - & - & - & - & - & 7689 & AK 10 & 2.0 \\
\hline AM 11 & 2.0 & - & - & - & - & - & - & 7530 & All 11 & 2.0 \\
\hline Als 12 & 2.0 & - & - & - & - & - & - & 7459 & AK 12 & 2.0 \\
\hline An 13 & 0.5 & - & - & - & - & - & - & 17513 & An 13 & 0.5 \\
\hline An 13 & 2.0 & - & - & - & - & - & - & 9807 & Af 13 & 2.0 \\
\hline AH IJ & 3.0 & - & - & - & - & - & - & 6114 & AN 13 & 3.0 \\
\hline An 13 & 4.0 & - & - & - & - & - & - & 7272 & Af $1 ?$ & 4.0 \\
\hline AH 13 & 4.5 & - & - & - & - & - & - & 6059 & AK $1:$ & 4.5 \\
\hline Af 13 & 5.0 & - & - & - & - & - & - & 8337 & An 1: & 5.0 \\
\hline An 14 & 2.0 & - & - & - & - & - & - & 10706 & An 14 & 2.0 \\
\hline AN 23 & 2.0 & - & - & - & - & - & - & 9555 & A. 23 & 2.0 \\
\hline All 24 & 2.0 & - & - & - & - & - & - & 8240 & Aั 24 & 2.0 \\
\hline All 25 & 2.0 & - & - & - & - & - & - & 7703 & A⿱ 25 & 2.0 \\
\hline Af 47 & 2.0 & - & - & - & - & - & - & 9970 & AY 47 & 2.0 \\
\hline An 48 & 2.0 & - & - & - & - & - & - & 9735 & A. 48 & 2.0 \\
\hline An 49 & 2.0 & - & - & - & - & - & - & 10788 & A.: 49 & 2.0 \\
\hline An 50 & 2.0 & - & - & - & - & - & - & 9690 & A: 50 & 2.0 \\
\hline An 51 & 2.0 & - & - & - & - & - & - & 9579 & At: 51 & 2.0 \\
\hline An 52 & 2.0 & - & - & - & 3.5 & - & 2.0 & 36215 & A. 52 & 2.0 \\
\hline A月 53 & 2.0 & - & - & - & - & - & - & 9661 & A* 53 & 2.0 \\
\hline Ah 54 & 2.0 & - & - & - & - & - & - & 19375 & Al: 54 & 2.0 \\
\hline AK 56 & 2.0 & - & - & - & - & - & - & 18986 & $A: 56$ & 2.0 \\
\hline An 57 & 2.0 & - & - & - & - & - & - & 9372 & AH 57 & 2.0 \\
\hline AH 58 & 2.0 & - & - & - & - & - & - & 94416 & AY: 58 & 2.0 \\
\hline A) 59 & 2.0 & - & - & - & - & - & - & 9293 & A. 59 & 2.0 \\
\hline Al 60 & 2.0 & - & - & - & - & - & - & 9532 & At: 60 & 2.0 \\
\hline AK 61 & 2.0 & - & - & - & - & - & - & 9560 & AK 61 & 2.0 \\
\hline An 62 & 2.0 & - & - & - & - & - & - & 7356 & An 62 & 2.0 \\
\hline An 63 & 2.0 & - & - & - & - & - & - & 11460 & An 63 & 2.0 \\
\hline Af 64 & 2.0 & - & - & - & - & - & - & 32072 & An 64 & 2.0 \\
\hline An 65 & 2.0 & - & - & - & - & - & - & 9135 & AM 65 & 2.0 \\
\hline All 66 & 2.0 & - & - & - & - & - & - & 9443 & AN 66 & 2.0 \\
\hline Al 67 & 2.0 & - & - & - & - & - & - & 20690 & Af 67 & 2.0 \\
\hline AH 68 & 2.0 & - & - & - & - & - & - & 8928 & AN 68 & 2.0 \\
\hline AH 69 & 2.0 & - & - & - & - & - & - & 8868 & AM 69 & 2.0 \\
\hline
\end{tabular}




\section{Esso Gasoline Station}

The 01d Ellenton Esso gasoline station is located at the intersection of South Carolina Rt. 125 and Main Street (SRP Rt.3), as shown on the Index Map (Figure 1). Using the same rational as discussed with respect to the Amoco station, a grid of 36 sites on 10 and $20 \mathrm{ft}$ centers was sampled for light hydrocarbons $\mathrm{C}_{1}-\mathrm{C}_{4}$, as shown on Figure 7. Results of these analyses are shown on Figures 8, 9 and 10, and the data are tabulated in Tables 5 and 6 .

On the same basis as considered for the Amoco station data, only 4 sites at the Esso station were found marginally anomalous in $\%$ butane, and only two of these had anomalous butane magnitudes. Methane magnitudes were likewise found to be normal. No evidence of underground objects was encountered. Based on these data, no soil samples were taken for gasoline range hydrocarbon analysis. 


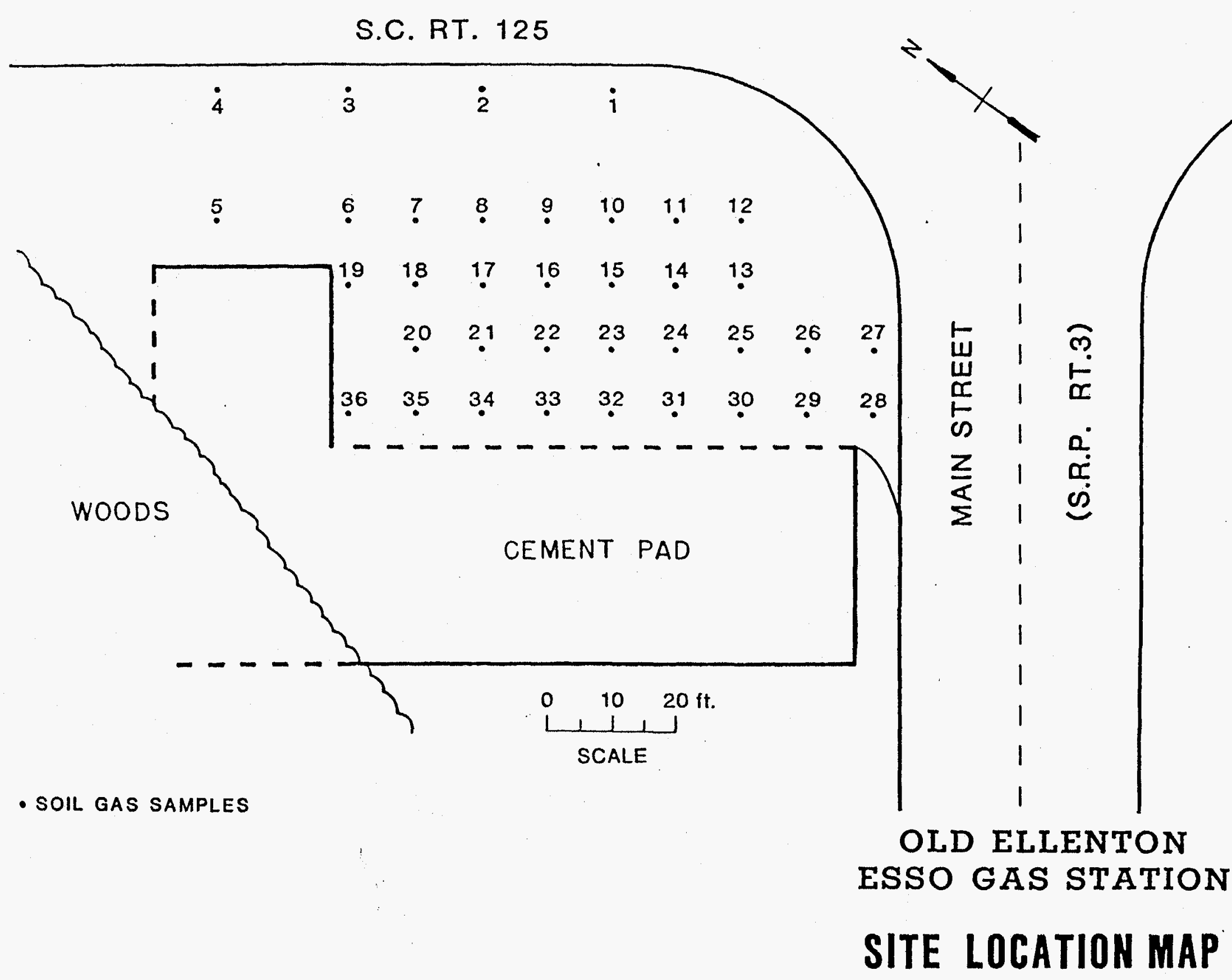

S.R.P.

NOVEMBER, 1986 


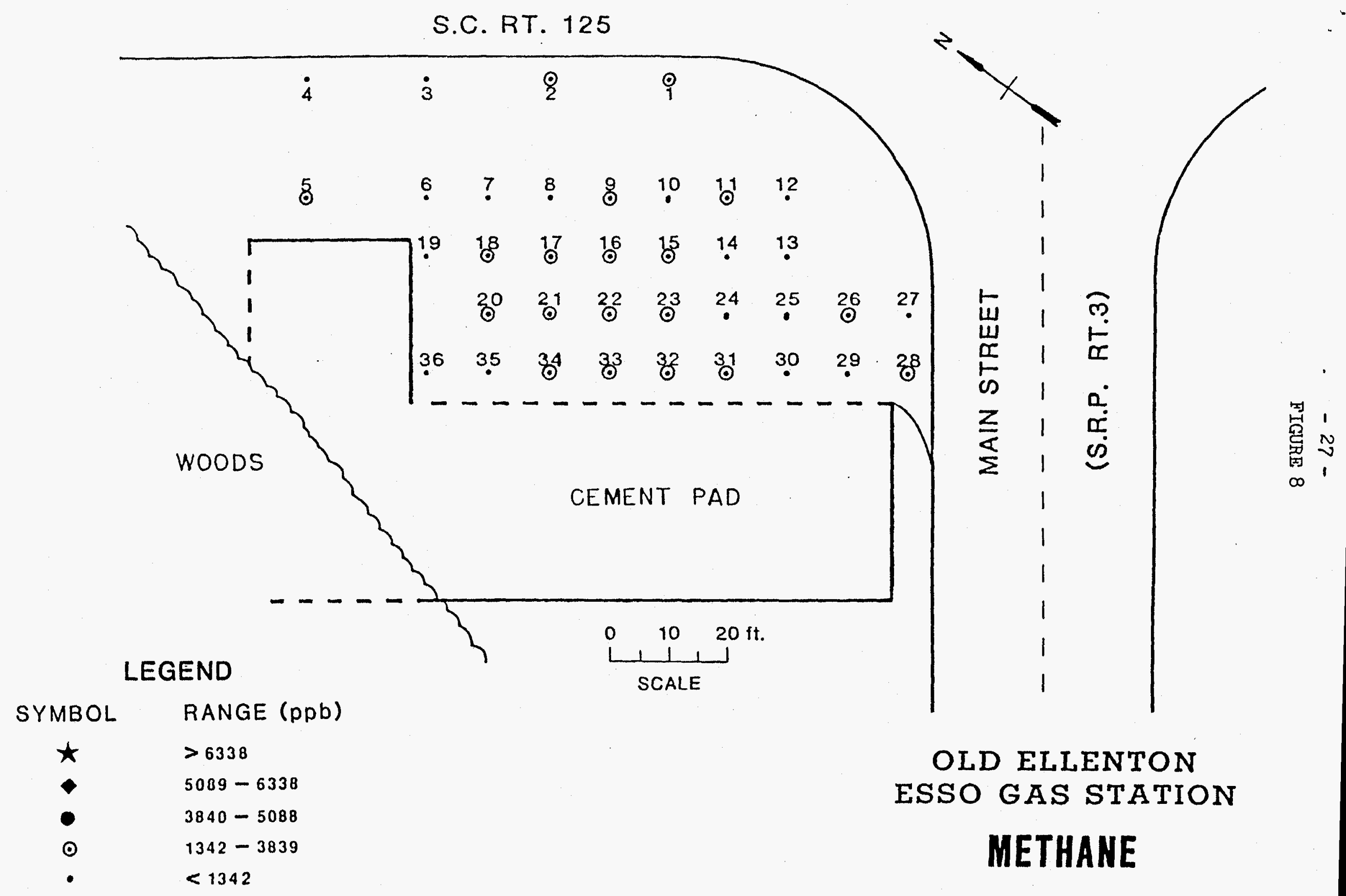

S.R.P.

NOVEMBER, 1986 

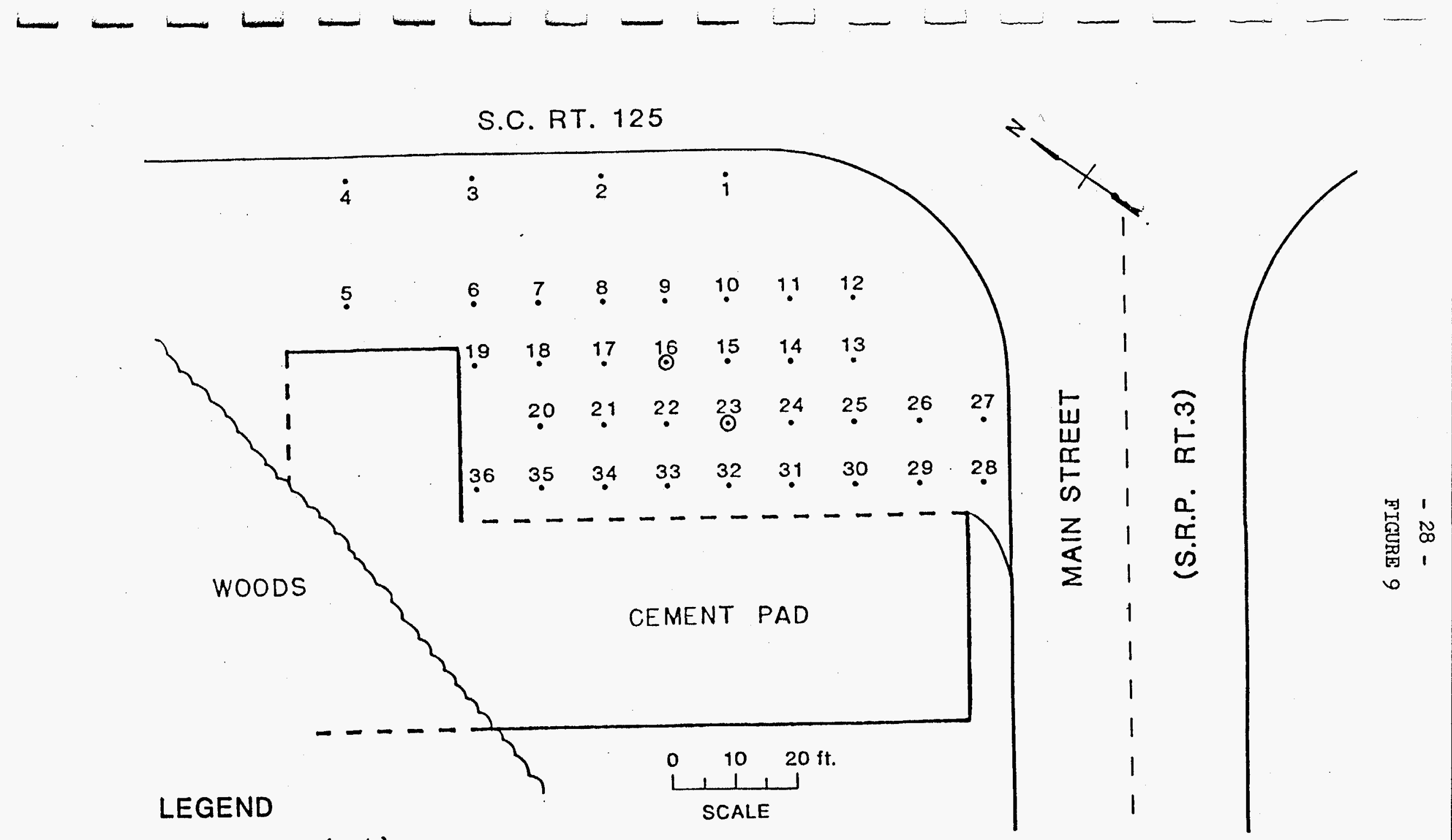

SYMBOL RANGE ( $p p b)$

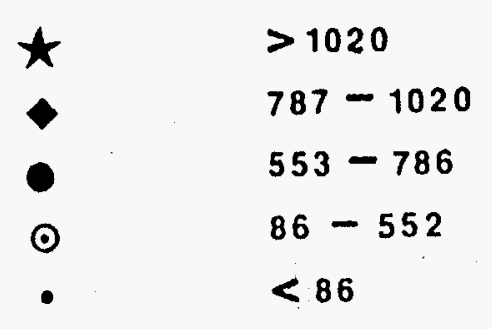

\author{
OLD ELLENTON \\ ESSO GAS STATION \\ BUTANE
}

S.R.P.

NOVEMBER, 1986 


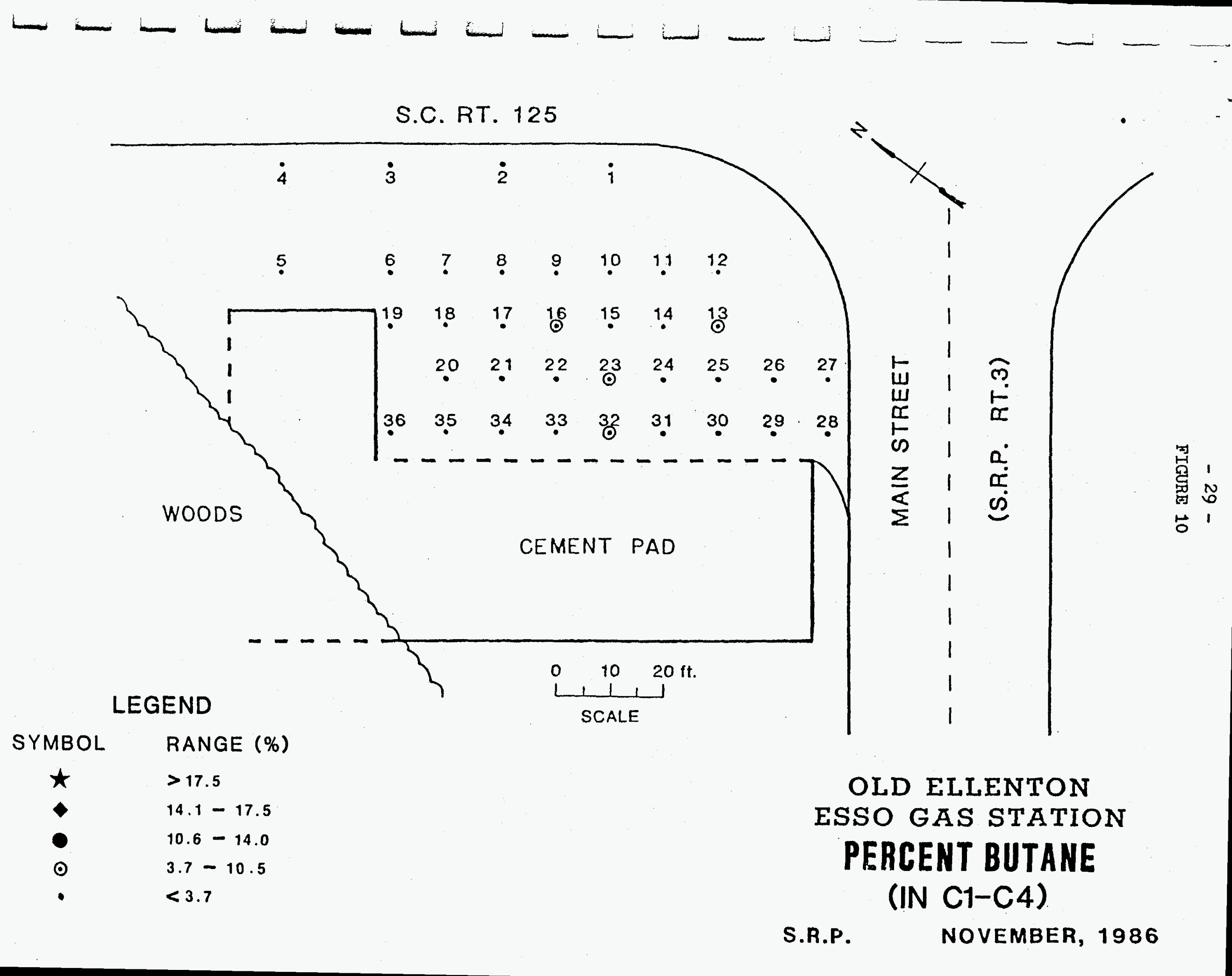


--- E. 1. DUPQNT DE MEKOURS CO. IMC., S.R.P., AIKEN, S.C. -..

--- OLD ELLENTON / ESSO STATION -- 3 FT. PROBE SURVEY, SEPT 1986 ----

-.-- SOIL GAS CONCENTRATIONS -..-

\begin{tabular}{|c|c|c|c|c|c|c|c|c|c|c|}
\hline SAMP & & $\begin{array}{r}\text { METHANE } \\
P P B\end{array}$ & $\begin{array}{r}\text { ETHANE } \\
\text { PPB }\end{array}$ & $\begin{array}{l}\text { PROPANE } \\
\text { PPB }\end{array}$ & $\begin{array}{c}\text { [-BUTARE } \\
\text { PPB }\end{array}$ & $\begin{array}{c}\text { H-BUtane E } \\
\text { PPE }\end{array}$ & $\begin{array}{c}\text { ETHYLENE PI } \\
\text { PPB }\end{array}$ & $\begin{array}{c}\text { PROPYLEME } \\
\text { PPB }\end{array}$ & SAYPLE & \\
\hline 50 & 1 & 2174 & 120 & 45 & 62 & 7 & 100 & 100 & so & \\
\hline SO & 2 & 1551 & 63 & 31 & 27 & - & 37 & 26 & 50 & 2 \\
\hline So & 3 & : j04. & 33 & 14 & 16 & - & 22 & 24 & So & 3 \\
\hline So & 4 & 957 & 37 & 14 & 22 & - & 25 & 23 & so & \\
\hline so & 5 & 1754 & 57 & 19 & 20 & - & 36 & 31 & so & \\
\hline SO & 6 & 1217 & 66 & 23 & 20 & - & 42 & 38 & So & \\
\hline so & 7 & 567 & 22 & 8 & - & - & 15 & - & so & \\
\hline SO & 8 & 1275 & 59 & 23 & 23 & - & 36 & 32 & so & \\
\hline So & 9 & 2058 & 141 & 53 & 67 & 13 & 111 & 110 & so & \\
\hline so & 10 & 1041 & 68 & 25 & 24 & - & 51 & 53 & So 1 & 10 \\
\hline so & 11 & 2203 & 115 & 46 & 41 & - & 90 & 75 & so 1 & 11 \\
\hline SO & 12 & 923 & 21 & 13 & - & - & 12 & - & so 1 & 12 \\
\hline 50 & 13 & 1297 & 52 & 24 & 66 & - & 62 & 46 & So 1 & 13 \\
\hline SO & 14 & 1116 & 53 & 22 & 31 & - & 40 & 62 & so 1 & 14 \\
\hline so & 15 & 1797 & 108 & 10 & 55 & - & 83 & 81 & so 1 & 15 \\
\hline SO & $1 t$ & 3261 & 226 & 82 & 229 & 19 & 190 & 196 & So 1 & 16 \\
\hline SO & $1:$ & 1391 & 71 & 24 & 36 & - & 45 & 50 & So 1 & 17 \\
\hline SD & 15 & 1406 & 61 & 27 & 27 & - & 39 & 38 & So 1 & 18 \\
\hline 50 & 15 & 1050 & 32 & 21 & - & - & 26 & 17 & So 1 & 19 \\
\hline so & $2 i$ & 1478 & 76 & 38 & 33 & - & 64 & 49 & SO 2 & 20 \\
\hline 50 & 21 & 1391 & 65 & 22 & 44 & - & 44 & 39 & So 2 & 21 \\
\hline SO & 22 & 1536 & 101 & 36 & 62 & - & 81 & 85 & So 2 & 22 \\
\hline So & $2 j$ & 1623 & 148 & 61 & 96 & 19 & 111 & 144 & So 2 & \\
\hline 50 & 24 & 1246 & 72 & 37 & 34 & - & 72 & 54 & So 2 & 24 \\
\hline so & $2^{5}$ & 971 & $4 !$ & 15 & 20 & - & 25 & 23 & so 2 & 23 \\
\hline SD & $2 i$ & 1624 & 37 & 12 & 13 & - & 22 & 17 & so 2 & 26 \\
\hline SO & $2 i$ & 1232 & 23 & 7 & - & - & 14 & - & So 2 & 27 \\
\hline So & 28 & 1594 & 33 & 11 & 27 & - & 20 & 15 & SO 2 & 28 \\
\hline 50 & 29 & 1162 & 34 & 33 & 20 & - & 40 & 41 & So 2 & 29 \\
\hline 50 & 30 & 1062 & 51 & 21 & 35 & - & 33 & 64 & So 3 & 30 \\
\hline so & 31 & 1609 & 89 & 23 & 39 & - & 61 & 48 & So 3 & 31 \\
\hline 50 & 32 & 1449 & 109 & 44 & 77 & - & 86 & 98 & So 3 & 32 \\
\hline 50 & 33 & 1913 & 106 & 37 & 49 & 6 & 82 & 67 & So 3 & 33 \\
\hline SO & 34 & 1348 & 88 & 40 & 17 & 6 & 90 & 74 & So 3 & \\
\hline so & 35 & 1109 & 66 & 51 & 26 & - & 73 & 65 & So 3 & 35 \\
\hline so & 36 & 990 & 28 & 34 & 12 & - & 37 & 23 & 503 & \\
\hline
\end{tabular}


--- E. I. DUPOHT DE MENOURS CO. INC., S.R.P., AIKER, S.C. ---

OLD ELLERTON / ESSO STATLON - 3 FT. PROBE SURVEY, SEPT 1986

--.- SOIL GAS PERCENTAGES ----

\begin{tabular}{|c|c|c|c|c|c|c|c|c|c|c|c|}
\hline SAMP & & $\begin{array}{l}\text { TOTAL } \\
\text { CI-CH }\end{array}$ & $\begin{array}{r}\text { METHANE } \\
\text { TCI }\end{array}$ & $\begin{array}{r}\text { ETHAKE } \\
2 \mathrm{C} 2\end{array}$ & $\begin{array}{r}\text { PROPANE } \\
\text { ICJ }\end{array}$ & $\begin{array}{l}\text { BUTANE } \\
\text { ZCA }\end{array}$ & $\begin{array}{c}\text { TOTAL } \\
\text { HYDCARB }\end{array}$ & $\begin{array}{r}\text { ETHYLENE } \\
?\end{array}$ & $\begin{array}{c}\text { PROPYLERE } \\
z\end{array}$ & \multicolumn{2}{|c|}{ SAMPLE } \\
\hline so & 1 & 2408 & 90.3 & 5.0 & 1.9 & 2.9 & 2608 & 3.8 & 3.8 & so & 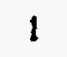 \\
\hline so & 2 & 1672 & 92.8 & 3.8 & 1.9 & 1.6 & 1735 & 2.1 & 1.5 & 50 & 2 \\
\hline so & 3 & 1367 & 95.1 & 2.4 & 1.0 & 1.2 & 1413 & 1.6 & 1.7 & 50 & 3 \\
\hline so & 4 & 1030 & 92.9 & 3.6 & 1.4 & 2.1 & 1078 & 2.3 & 2.1 & 50 & 4 \\
\hline 50 & 5 & 1850 & 94.8 & 3.1 & 1.0 & 1.1 & 1917 & 1.9 & 1.6 & 50 & 5 \\
\hline SE & 6 & 1326 & 91.8 & 5.0 & 1.7 & 1.5 & 1406 & 3.0 & 2.7 & 50 & , \\
\hline 50 & 7 & 597 & 95.0 & 3.7 & 1.3 & 0.0 & 612 & 2.5 & 0.0 & so & \\
\hline 50 & 8 & 1380 & 92.4 & 4.3 & 1.7 & 1.7 & 1448 & 2.5 & 2.2 & so & 8 \\
\hline SD & 9 & 2332 & 88.3 & 6.0 & 2.3 & 3.4 & 2553 & 4.3 & 4.3 & so & \\
\hline 50 & 10 & 1158 & 89.9 & 5.9 & 2.2 & 2.1 & 1262 & 4.0 & 4.2 & so & 10 \\
\hline SD & 11 & 2405 & 91.6 & 4.8 & 1.9 & 1.7 & 2570 & 3.5 & 2.9 & so & 11 \\
\hline SO & 12 & 957 & 96.4 & 2.2 & 1.4 & 0.0 & 969 & 1.2 & 0.0 & so & 12 \\
\hline 50 & 13 & 1439 & 90.1 & 3.6 & 1.7 & 4.6 & 1547 & 4.0 & 3.0 & so & 13 \\
\hline so & 11 & 1222 & 91.3 & 4.3 & 1.8 & 2.5 & 1324 & 3.0 & 4.7 & SO & 14 \\
\hline 50 & 15 & 2000 & 89.9 & 5.4 & 2.0 & 2.8 & 2164 & 3.8 & 3.7 & S0 & 15 \\
\hline 50 & 16 & 3817 & 95.1 & 5.9 & 2.1 & 6.5 & 4203 & 4.5 & 4.7 & so & 16 \\
\hline SO & 17 & 1522 & 91.4 & 4.7 & 1.6 & 2.4 & 1617 & 2.8 & 3.1 & SD & 17 \\
\hline 50 & 18 & 1521 & 92.4 & 4.0 & 1.8 & 1.8 & 1598 & 2.4 & 2.4 & so & 18 \\
\hline So & 19 & 1103 & 95.2 & 2.9 & 1.9 & 0.0 & 1146 & 2.3 & 1.5 & 58 & 19 \\
\hline so & 20 & 1625 & 91.0 & 4.7 & 2.3 & 2.0 & 1738 & 3.7 & 2.8 & So & 20 \\
\hline 50 & 21 & 1522 & 91.4 & 4.3 & 1.4 & 2.9 & 1605 & 2.7 & 2.4 & so & 21 \\
\hline SO & 22 & 1735 & 88.5 & 5.8 & 2.1 & 3.6 & 1901 & 4.3 & 4.5 & SO & 22 \\
\hline SD & 23 & 1947 & 83.4 & 7.6 & 3.1 & 5.9 & 2205 & 5.2 & 6.5 & so & 23 \\
\hline 50 & 24 & 1389 & 89.7 & 5.2 & 2.7 & 2.4 & 1515 & 4.8 & 3.6 & So & 24 \\
\hline S0 & 25 & 1047 & 92.7 & 3.9 & 1.4 & 1.9 & 1095 & 2.3 & 2.1 & so & 25 \\
\hline 50 & $2 t$ & 1686 & 96.3 & 2.2 & 0.7 & 0.8 & 1725 & 1.3 & 1.0 & 50 & 26 \\
\hline so & 27 & 1282 & 97.6 & 1.8 & 0.6 & 0.0 & 1276 & 1.1 & 0.0 & 50 & 27 \\
\hline SO & 28 & 1665 & 95.7 & 2.0 & 0.7 & 1.6 & 1700 & 1.2 & 0.9 & 50 & 28 \\
\hline SO & 29 & 1249 & 93.0 & 2.7 & 2.6 & 1.6 & 1330 & 3.0 & 3.1 & so & 29 \\
\hline So & 30 & 1169 & 90.8 & 4.1 & 1.8 & 3.0 & 1266 & 2.6 & 5.1 & SO & 30 \\
\hline 50 & 31 & 1760 & 91.4 & 5.1 & 1.3 & 2.2 & 1869 & 3.3 & 2.6 & SO & 31 \\
\hline so & 32 & 1679 & 86.3 & 6.5 & 2.6 & 4.6 & 1863 & 4.6 & 5.3 & so & 32 \\
\hline 50 & 33 & 2111 & 90.6 & 5.0 & 1.8 & 2.6 & 2260 & 3.6 & 3.0 & so & 33 \\
\hline SO & 34 & 1529 & 88.2 & 5.8 & 2.6 & 3.5 & 1693 & 5.3 & 4.4 & so & 3 \\
\hline so & 35 & 1252 & 88.6 & 5.3 & 4.1 & 2.1 & 1390 & 5.3 & 4.7 & so & 35 \\
\hline 50 & 36 & 1064 & 93.0 & 2.6 & 3.2 & 1.1 & 1124 & 3.3 & 2.0 & so & 36 \\
\hline
\end{tabular}


Oil Company Site

The oil company site is located near the intersection of Railroad Avenue and Main Street (SRP Rt. 3) as shown on the Index Map on Figure 1. Very little information regarding activity at this site was available. A total of 67 sites were sampled at a depth of $3 \mathrm{ft}$ for light hydrocarbons. Locations of these sites are shown on Figure 11. Data resulting from sample analysis is shown on Figures 12, 13 and 14, and is tabulated in Tables 7 and 8 .

A large methane anomaly was encountered at Site 37 , as shown on Figure 12. Based on composition, this is probably methane generated from biological activity. Other high methane sites were found in close proximity. No probable source of this methane is known to us.

Several sites were found anomalous with respect to percent butane (see Figure 14), suggesting the presence of gasoline. Based on butane magnitudes, these sites are only marginally anomalous (see Figure 13), suggesting that the level of contamination here is smaller than at the Amoco station.

At five sites, soil samples were taken for gasoline range hydrocarbon analysis. No gasoline range hydrocarbons were found above the minimum detection level (see Table 1). 


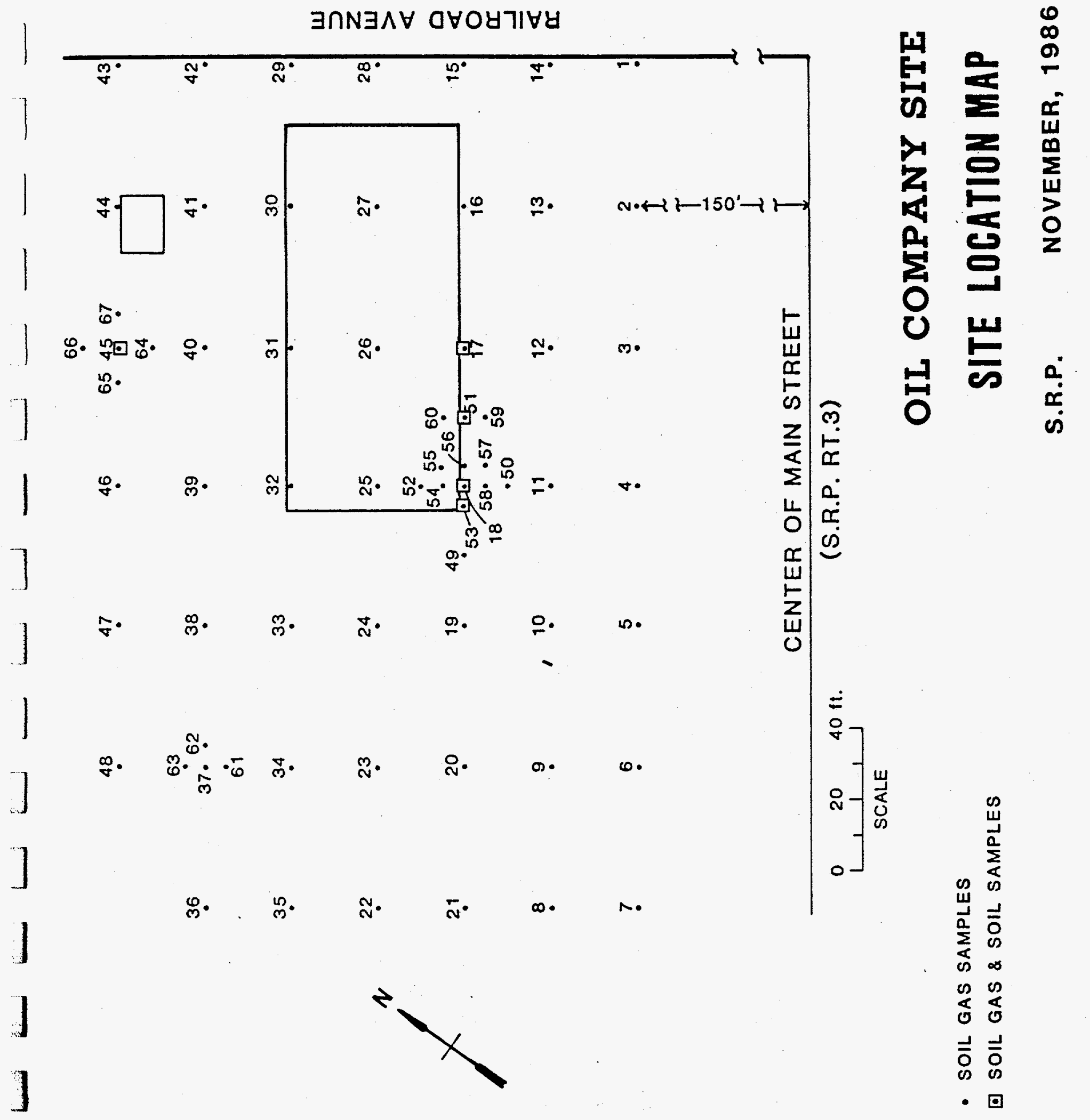




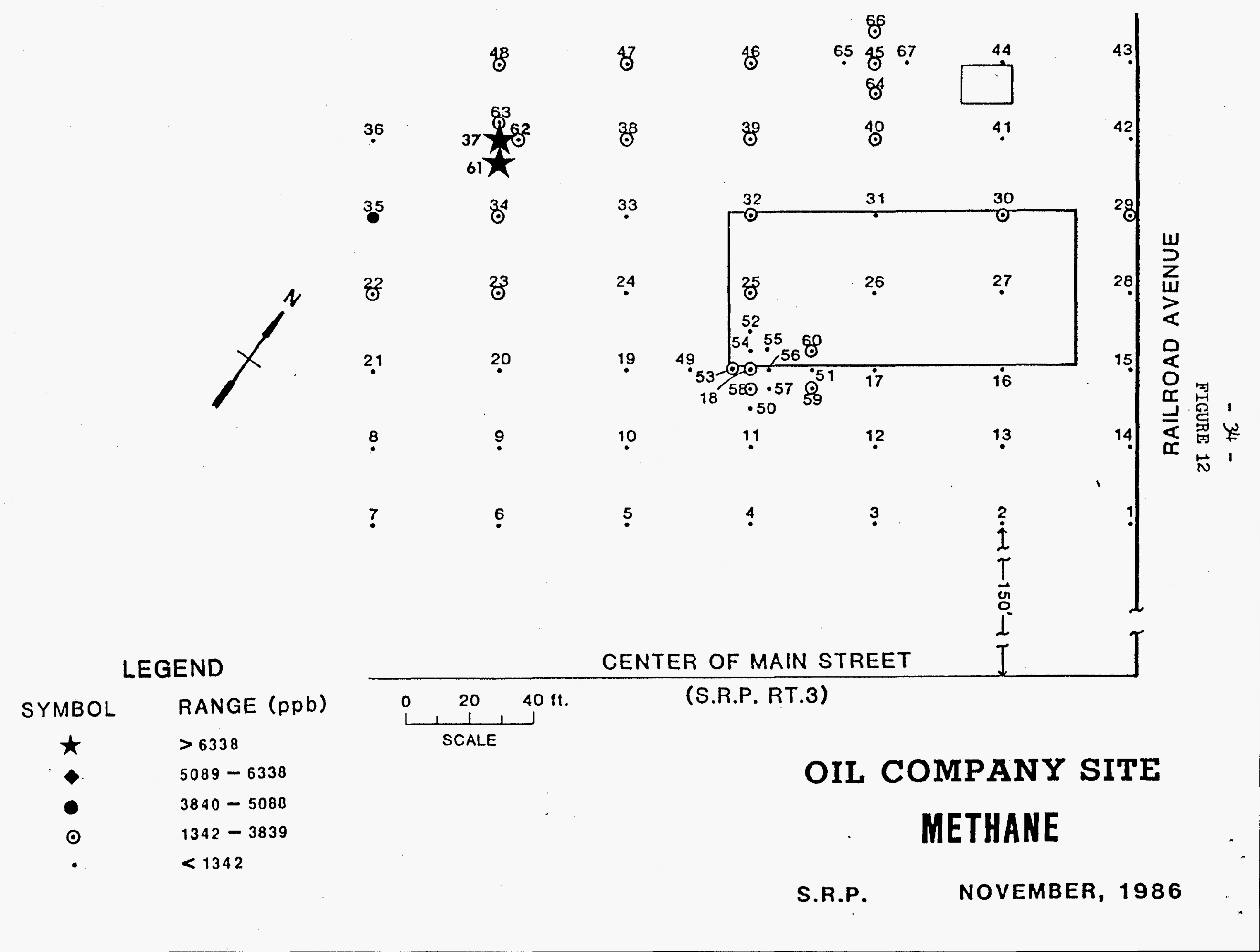




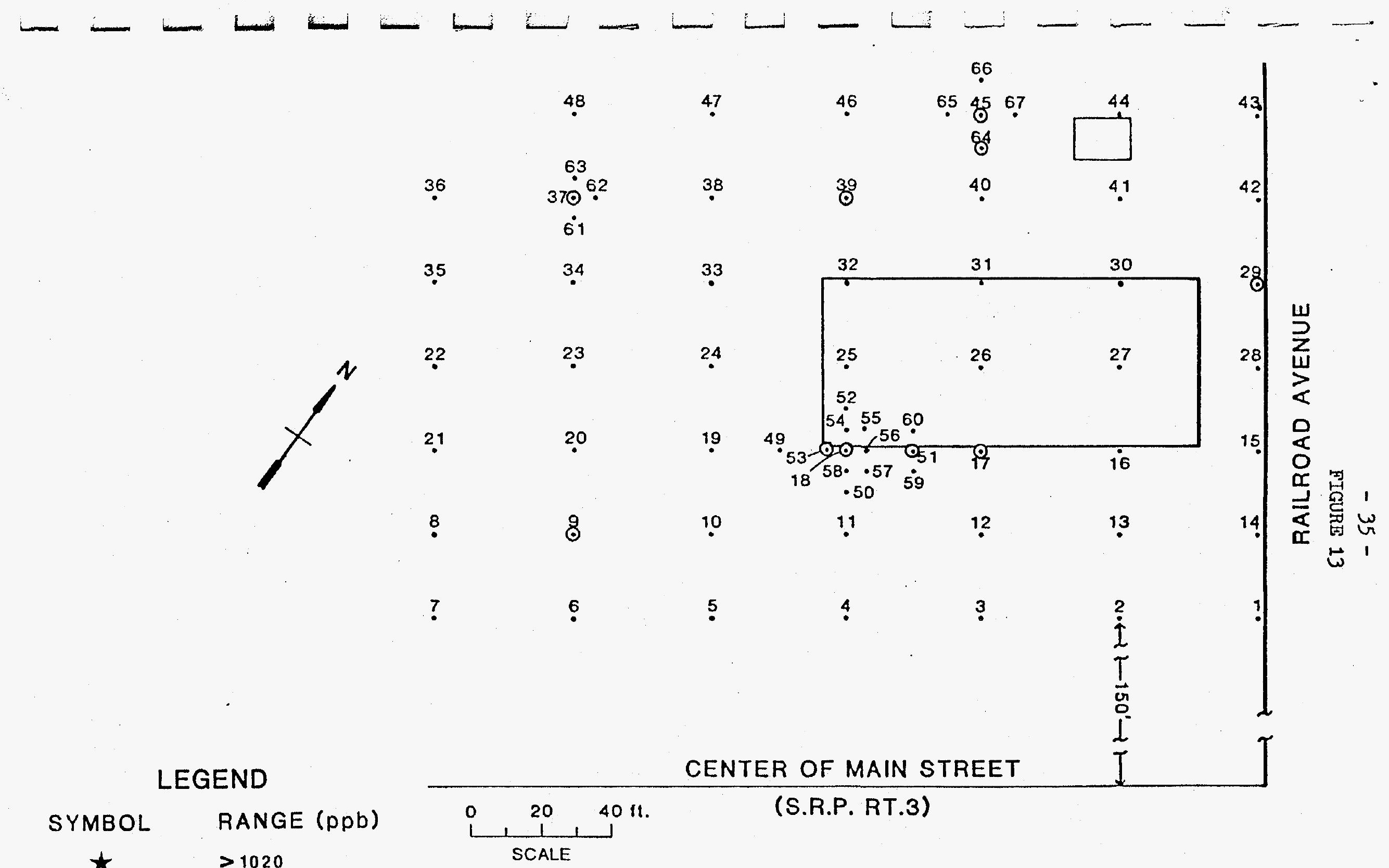

$\begin{array}{ll}\star & >1020 \\ \bullet & 787-1020 \\ \bullet & 553-786 \\ \odot & 86-552 \\ \cdot & <86\end{array}$

OIL COMPANY SITE BUTANE 


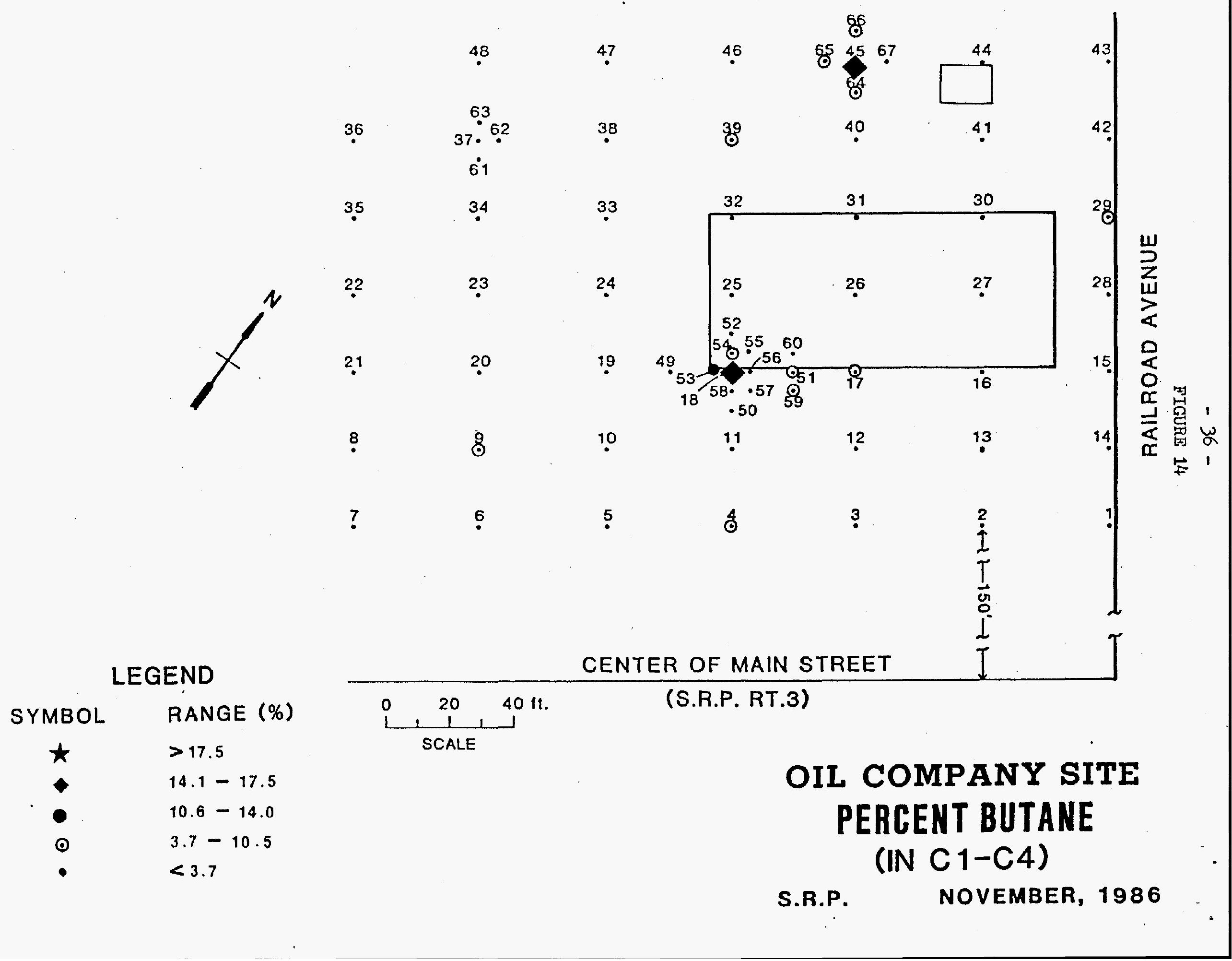


-.- E. I. DUPONT DE MEMOURS CO. INC., S.R.P., AIKEN, S.C. ---

--- OLD ELLENTOK / OIL CO. -- J FI. PROBE SURVEY, SEPT 1986 -..-

---- SOIL GAS CONCENTRATIONS ----

SAMPLE METHANE ETHANE PROPANE I-BUTANE N-BUtAME ETHYLEME PROPYLEME SAMPLE

\begin{tabular}{|c|c|c|c|c|c|c|c|c|c|c|}
\hline \multicolumn{2}{|c|}{1} & PPB & PPB & PPB & PPB & PPE & PPB & PPB & & 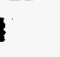 \\
\hline oc & 1 & 1055 & 35 & 14 & 22 & - & 30 & 27 & OC & 1 \\
\hline$O C$ & 2 & 796 & 16 & - & - & - & 11 & - & OC & 2 \\
\hline OC & 3 & 513 & 11 & - & - & - & 14 & - & OC & 3 \\
\hline OC & 4 & 668 & 24 & 14 & 72 & - & 16 & 12 & OC & 4 \\
\hline$O C$ & 5 & 637 & 23 & 11 & 18 & - & 16 & 12 & $O C$ & 5 \\
\hline OC & 6 & 658 & 27 & 12 & - & - & 16 & 17 & BS & 6 \\
\hline OC & 7 & 666 & 25 & 9 & - & - & 16 & 14 & OC & 7 \\
\hline OC & 8 & 963 & 41 & 21 & 21 & - & 25 & 23 & $0 C$ & 8 \\
\hline$O C$ & 9 & 1333 & 71 & 84 & 116 & 17 & 31 & 36 & $O C$ & 9 \\
\hline$O C$ & 10 & 1232 & 33 & 15 & 17 & - & 23 & 21 & OC & 10 \\
\hline OC & 11 & 910 & 26 & 10 & 18 & - & 19 & 15 & OC & 11 \\
\hline$O C$ & 12 & 859 & 17 & 11 & - & - & 13 & - & OC & 12 \\
\hline$O C$ & 13 & 942 & 34 & 20 & - & - & 17 & 14 & $O C$ & 13 \\
\hline$O C$ & 14 & 1188 & 28 & 17 & 22 & - & 24 & 21 & OC & 14 \\
\hline$O C$ & 15 & 1181 & 29 & 16 & 19 & - & 38 & 30 & OC & 15 \\
\hline$O C$ & 16 & 1304 & 46 & 20 & 14 & - & 26 & 22 & $O C$ & 16 \\
\hline$O C$ & 17 & 1130 & 30 & 80 & 77 & 41 & 18 & 9 & $O C$ & 17 \\
\hline OC & 18 & 1449 & 53 & 64 & 248 & 39 & 45 & 35 & OC & 18 \\
\hline$O C$ & $18 R$ & 1280 & 37 & 29 & 139 & 19 & 39 & 33 & OC & $18 R$ \\
\hline$O C$ & 19 & 1281 & 39 & 17 & 17 & - & 22 & 21 & OC & 19 \\
\hline OC & 20 & 1000 & 28 & 33 & 33 & - & 22 & 12 & OC & 20 \\
\hline OC & 21 & 1181 & 58 & 47 & 47 & - & 31 & 30 & oc & 21 \\
\hline OC & 22 & 1768 & 88 & 44 & 44 & - & 66 & 65 & oc & 22 \\
\hline OC & 23 & 2029 & 118 & 57 & 57 & - & 67 & 70 & $O C$ & 23 \\
\hline$O C$ & 24 & 1115 & 37 & 24 & 24 & - & 22 & 19 & oc & 24 \\
\hline OC. & 25 & 2188 & 48 & 38 & 38 & - & 39 & 10 & OC & 25 \\
\hline$D C$ & 26 & 917 & 34 & 19 & 19 & - & 21 & 23 & $O C$ & 26 \\
\hline OC & 27 & 1260 & 43 & 17 & 17 & - & 23 & 16 & oc & 27 \\
\hline OC & 28 & 1041 & 43 & 20 & 20 & - & 41 & 27 & OC & 28 \\
\hline$O C$ & 29 & 3203 & 357 & 160 & 284 & 70 & 447 & 425 & oc & 29 \\
\hline$O C$ & 30 & 1405 & 42 & 16 & 20 & - & 29 & 29 & OC & 30 \\
\hline OC & 31 & 1275 & 52 & 18 & 27 & - & 51 & 42 & oc & 31 \\
\hline OC & 32 & 1594 & 32 & 11 & 15 & - & 26 & 18 & OC & 32 \\
\hline OC & 33 & 1287 & 50 & 18 & 20 & - & 26 & 25 & OC & 33 \\
\hline oc & 34 & 2652 & 126 & 57 & 72 & - & 86 & 76 & oC & 34 \\
\hline or & 35 & 4710 & 246 & 107 & 25 & 39 & 305 & 297 & OC & 35 \\
\hline OC & 36 & 1043 & 23 & 20 & - & - & 14 & - & oc & 36 \\
\hline OC & 37 & 49468000 & \pm & 175 & 111 & 72 & 115 & 226 & OC & 37 \\
\hline OC & 38 & 2029 & 61 & 22 & 30 & - & 40 & 42 & $D C$ & 38 \\
\hline oc & 39 & 2899 & 100 & 40 & 153 & 26 & 78 & 71 & OC & 39 \\
\hline OC & 40 & 1478 & 52 & 19 & 33 & - & 35 & 36 . & OC & 40 \\
\hline oc & 41 & 1295 & 28 & 12 & 14 & - & 18 & 16 & oc & 41 \\
\hline oc & 42 & 1290 & 65 & 24 & 38 & - & 19 & 16 & $O C$ & 42 \\
\hline OC & 13 & 1127 & 46 & 16 & 16 & - & 27 & 23 & OC & 43 \\
\hline OC & 44 & 913 & 11 & 22 & 18 & - & 26 & 29 & OC & 44 \\
\hline$O C$ & 45 & 1459 & 53 & 26 & 284 & 39 & 34 & 34 & $O C$ & 45 \\
\hline oc & $15 R$ & 1289 & 44 & 18 & 75 & - & 23 & 27 & $O C$ & $45 R$ \\
\hline OC & 46 & 1667 & 82 & 29 & 36 & 6 & 44 & 42 & $O C$ & 46 \\
\hline OC & 17 & 2493 & 44 & 20 & 19 & - & 33 & 32 & OC & 47 \\
\hline
\end{tabular}


E. I. DUPONT DE MEROURS CO. IMC., S.R.P., AIKEN, S.C. ----

-.-- OLD ELLENTON / OIL CO. -- 3 FT. PROBE SURVEY, SEPT 1986 -..-

--- SOIL GAS CONCENTRATIONS ----

\begin{tabular}{|c|c|c|c|c|c|c|c|c|c|c|}
\hline & PLE & $\begin{array}{r}\text { METHANE } \\
\text { PPB }\end{array}$ & $\begin{array}{r}\text { ETHANE } \\
\text { PPB }\end{array}$ & $\begin{array}{r}\text { PROPANE } \\
\text { PPB }\end{array}$ & $\begin{array}{c}\text { 1-BUTANE } \\
\text { PPB }\end{array}$ & $\begin{array}{c}\text { N-BUTANE } \\
\text { PPB }\end{array}$ & $\begin{array}{r}\text { ETHYLENE } \\
\text { PPB }\end{array}$ & $\begin{array}{c}\text { PROPYLEME } \\
\text { PPB }\end{array}$ & SAMP & PLE \\
\hline OC & 48 & 1696 & 87 & 30 & 30 & - & 46 & 45 & OC & 48 \\
\hline$O C$ & 49 & 1152 & 57 & 25 & 10 & - & 37 & 31 & OC & 19 \\
\hline OC & 50 & 1275 & 33 & 12 & 17 & - & 24 & 22 & oc & 50 \\
\hline OC & 51 & 1072 & 45 & 47 & 80 & 33 & 29 & 24 & OC & 51 \\
\hline OC & 52 & 899 & 33 & 19 & 21 & - & 20 & 12 & $D C$ & 52 \\
\hline$O C$ & 53 & 2340 & 95 & 151 & 296 & 88 & 88 & 79 & $O C$ & 53 \\
\hline OC & 54 & 1115 & 29 & 16 & 76 & - & 32 & 28 & $D C$ & 54 \\
\hline$O C$ & 55 & 1112 & 37 & 18 & 38 & - & 24 & 23 & $D C$ & 55 \\
\hline OC & 56 & 1168 & 15 & 32 & 30 & - & 12 & 12 & $O C$ & 56 \\
\hline OC & 57 & 1209 & 39 & 15 & 34 & - & 23 & 23 & OC & 57 \\
\hline oc & 58 & 1522 & 44 & 17 & 29 & - & 28 & 33 & OC & $5 t$ \\
\hline OC & 59 & 1371 & 42 & 17 & 60 & - & 24 & 25 & $O C$ & 5 \\
\hline$O C$ & 60 & 1680 & 59 & 23 & 26 & - & 37 & 31 & $O C$ & $t:$ \\
\hline$O C$ & 61 & 12957 & 84 & 39 & 26 & - & 51 & 50 & $O C$ & $\varepsilon:$ \\
\hline$B C$ & 62 & 1663 & 75 & 34 & 35 & - & 47 & 50 & OC & $t_{-}^{-}$ \\
\hline$D C$ & 63 & 2405 & 15 & 9 & 16 & - & 13 & - & OC & $: j$ \\
\hline OC & 64 & 1517 & 67 & 29 & 102 & - & 43 & 18 & $O C$ & : 4 \\
\hline OC & 65 & 1311 & 65 & 27 & 69 & - & 65 & 40 & DC & .5 \\
\hline OC & 66 & 1431 & 59 & 22 & 59 & - & 32 & 38 & OC & 36 \\
\hline$D C$ & 67 & 1204 & 34 & 13 & 19 & - & 18 & 20 & OC & 67 \\
\hline
\end{tabular}


--- E. I. DUPONT DE NEMOURS CO. INC., S.R.P., AIKEN, S.C. ---

--- OLD ELLENTON / OIL CO. - 3 FT. PROBE SURVEY, SEPT 1988 -

-.- SOIL GAS PERCENTAGES --..-

\begin{tabular}{|c|c|c|c|c|c|c|c|c|c|c|c|}
\hline SAMF & & $\begin{array}{l}\text { TOTAL } \\
\text { CI-C4 }\end{array}$ & $\begin{array}{r}\text { METHANE } \\
\text { ZCI }\end{array}$ & $\begin{array}{r}\text { ETHANE } \\
\text { IC2 }\end{array}$ & $\begin{array}{r}\text { PROPANE } \\
\text { 2C3 }\end{array}$ & $\begin{array}{r}\text { BUTANE } \\
2 \mathrm{CA}\end{array}$ & $\begin{array}{l}\text { TOTAL } \\
\text { HYDCARB }\end{array}$ & $\begin{array}{r}\text { ETHYLEME } \\
2\end{array}$ & $\begin{array}{c}\text { PYLENE } \\
\text { I }\end{array}$ & SAMI & \\
\hline$D C$ & 1 & 793 & 93.2 & 4.8 & 2.0 & 0.0 & 876 & 4.7 & 4.8 & $O C$ & 1 \\
\hline$O C$ & 2 & 812 & 98.0 & 2.0 & 0.0 & 0.0 & 823 & 1.3 & 0.0 & $B C$ & 2 \\
\hline OC & 3 & 524 & 97.9 & 2.1 & 0.0 & 0.0 & 538 & 2.6 & 0.0 & OC & 3 \\
\hline$O C$ & 1 & 778 & 85.9 & 3.1 & 1.8 & 9.3 & 806 & 2.0 & 1.5 & DC & 4 \\
\hline OC & 5 & 689 & 92.5 & 3.3 & 1.6 & 2.6 & 717 & 2.2 & 1.7 & OC & 5 \\
\hline$O C$ & 6 & 697 & 94.1 & 3.9 & 1.7 & 0.0 & 730 & 2.2 & 2.3 & $O C$ & 6 \\
\hline OC & 7 & 700 & 95.1 & 3.6 & 1.3 & 0.0 & 730 & 2.2 & 1.9 & $D C$ & 7 \\
\hline ac & 8 & 1046 & 92.1 & 3.9 & 2.0 & 2.0 & 1094 & 2.3 & 2.1 & $D C$ & 8 \\
\hline QC & 9 & $165 !$ & 80.7 & 1.3 & 5.1 & 9.9 & 1718 & 1.8 & 2.1 & OC & 9 \\
\hline $\mathrm{OC}$ & 10 & 1297 & 95.0 & 2.5 & 1.2 & 1.3 & 1341 & 1.7 & 1.6 & OC & 10 \\
\hline$D C$ & 11 & 964 & 94.4 & 2.7 & 1.0 & 1.9 & 998 & $1 . .^{5}$ & 1.5 & OC & 11 \\
\hline OC & 12 & 887 & 96.8 & 1.9 & 1.2 & 0.0 & 900 & 1.: & 0.0 & OC & 12 \\
\hline OC & 13 & 996 & 94.6 & 3.4 & 2.0 & 0.0 & 1027 & 1. & 1.4 & OC & 13 \\
\hline OC & 14 & 1255 & 94.7 & 2.2 & 1.4 & 1.8 & 1300 & 1.3 & 1.6 & OC & 14 \\
\hline$O C$ & 15 & 1244 & 94.9 & 2.3 & 1.3 & 1.4 & 1312 & 27 & 2.3 & OC & 15 \\
\hline OC & 10 & 1384 & 94.2 & 3.3 & 1.4 & 1.0 & 1432 & 18 & 1.5 & DC & 16 \\
\hline$O C$ & 17 & 1358 & 83.2 & 2.2 & 5.9 & 8.7 & 1385 & 13 & 0.6 & OC & 17 \\
\hline OC & 18 & 1853 & 78.2 & 2.9 & 3.5 & 15.5 & 1933 & $\therefore .3$ & 1.8 & DC & 18 \\
\hline$O C$ & $18 R$ & 1504 & 85.1 & 2.5 & 1.9 & 10.5 & 1576 & $\therefore .5$ & 2.1 & OC & $18 R$ \\
\hline$O C$ & 19 & 1334 & 94.5 & 2.9 & 1.3 & 1.3 & 1377 & $\therefore .6$ & 1.5 & OC & 19 \\
\hline OC & 20 & 1094 & 91.4 & 2.6 & 3.0 & 3.0 & 1128 & 2.0 & 1.1 & OC & 20 \\
\hline$D C$ & 21 & 1333 & 88.6 & 4.4 & 3.5 & 3.5 & 1394 & 2.2 & 2.2 & OC & 21 \\
\hline$O C$ & 22 & 1944 & 90.9 & 4.5 & 2.3 & 2.3 & 2075 & 3.2 & 3.1 & $O C$ & 22 \\
\hline OC & 23 & 2261 & 89.7 & 5.2 & 2.5 & 2.5 & 2398 & 2.8 & 2.9 & OC & 23 \\
\hline OC & 24 & 1200 & 92.9 & 3.1 & 2.0 & 2.0 & 1241 & 1.8 & 1.5 & $O C$ & 24 \\
\hline$O C$ & 25 & 2312 & 94.6 & 2.1 & 1.6 & 1.6 & 2391 & 1.6 & 1.7 & $O C$ & 25 \\
\hline OC & 26 & 989 & 92.7 & 3.4 & 1.9 & 1.9 & 1033 & 2.0 & 2.2 & OC & 26 \\
\hline DC & 27 & 1337 & 94.2 & 3.2 & 1.3 & 1.3 & 1376 & 1.7 & 1.2 & OC & 27 \\
\hline$D C$ & 28 & 1124 & 92.6 & 3.8 & 1.8 & 1.8 & 1192 & 3.4 & 2.3 & OC & 28 \\
\hline$D C$ & 29 & 4074 & 78.6 & 8.8 & 3.9 & 8.7 & 1946 & 9.0 & 8.6 & OC & 29 \\
\hline OC & 30 & 1483 & 94.7 & 2.8 & 1.1 & 1.3 & 1541 & 1.9 & 1.9 & OC & 30 \\
\hline OC & 31 & 1372 & 92.9 & 3.8 & 1.3 & 2.0 & 1465 & 3.5 & 2.9 & OC & 31 \\
\hline$O C$ & 32 & 1652 & 96.5 & 1.9 & 0.7 & 0.9 & 1696 & 1.5 & 1.1 & oC & 32 \\
\hline OC & $3 \mathfrak{3}$ & 1375 & 93.6 & 3.6 & 1.3 & 1.5 & 1426 & 1.8 & 1.8 & OC & 33 \\
\hline OC & 34 & 2907 & 91.2 & 4.3 & 2.0 & 2.5 & 3069 & 2.8 & 2.5 & oc & 34 \\
\hline$O C$ & 35 & 5127 & 91.9 & 4.8 & 2.1 & 1.2 & 5729 & 5.3 & 5.2 & DC & 35 \\
\hline OC & 36 & 1086 & 96.0 & 2.1 & 1.8 & 0.0 & 1100 & 1.3 & 0.0 & DC & 36 \\
\hline$O C$ & 37 & 9468358 & 100.0 & 0.0 & 0.0 & 0.0 & 49468699 & 0.0 & 0.0 & DC & 37 \\
\hline OC & 38 & 2142 & 94.7 & 2.8 & 1.0 & 1.4 & 2224 & 1.8 & 1.9 & OC & 38 \\
\hline OC & 39 & 3218 & 90.1 & 3.1 & 1.2 & 5.6 & 3367 & 2.3 & 2.1 & OC & 39 \\
\hline OC & 40 & 1582 & 93.1 & 3.3 & 1.2 & 2.1 & 1653 & 2.1 & 2.2 & DC & 40 \\
\hline$B C$ & 41 & 1319 & 96.0 & 2.1 & 0.9 & 1.0 & 1383 & 1.3 & 1.2 & OC & 41 \\
\hline OC & 42 & 1417 & 91.0 & 4.6 & 1.7 & 2.7 & 1512 & 3.2 & 3.0 & OC & 42 \\
\hline OC & 43 & 1205 & 93.5 & 3.8 & 1.3 & 1.3 & 1255 & 2.2 & 1.8 & OC & 43 \\
\hline oc & 44 & 994 & 91.9 & 4.1 & 2.2 & 1.8 & 1049 & 2.5 & 2.8 & OC & 44 \\
\hline OC & 45 & 1861 & 78.4 & 2.8 & 1.4 & 17.4 & 1929 & 1.8 & 1.8 & OC & 45 \\
\hline OC & 45R & 1426 & 90.4 & 3.1 & 1.3 & 5.3 & 1476 & 1.6 & 1.8 & OC & $45 R$ \\
\hline OC & 46 & 1820 & 91.6 & 4.5 & 1.6 & 2.3 & 1906 & 2.3 & 2.2 & OC & 46 \\
\hline OC & 47 & 2576 & 96.8 & 1.7 & 0.8 & 0.7 & 2641 & 1.2 & 1.2 & OC & 47 \\
\hline
\end{tabular}


--- E. I. DUPONT DE MENOURS CO. INC., S.R.P., AIKEN, S.C. --.-

--- DLD ELLEXTON / OIL CO. -- 3 FT. PROBE SURVEY, SEPT 1986 ---

--- SOIL GAS PERCENTAGES ----

\begin{tabular}{|c|c|c|c|c|c|c|c|c|c|c|c|}
\hline \multicolumn{2}{|c|}{$\begin{array}{c}\text { SAMPLE } \\
\vdots\end{array}$} & $\begin{array}{l}\text { TOTAL } \\
\text { CI-CA }\end{array}$ & $\begin{array}{r}\text { METHANE } \\
\text { ZCI }\end{array}$ & $\begin{array}{r}\text { ETHANE } \\
\text { ZC2 }\end{array}$ & $\begin{array}{r}\text { PRDPANE } \\
\text { ZCJ }\end{array}$ & $\begin{array}{r}\text { BUTANE } \\
\text { ZCA }\end{array}$ & $\begin{array}{l}\text { TOTAL } \\
\text { HYDCARB }\end{array}$ & ETHYLENE PROPYLENE & $\begin{array}{c}\text { PYLENE } \\
I\end{array}$ & \multicolumn{2}{|c|}{ SAMPLE } \\
\hline oc & 48 & 1843 & 92.0 & 4.7 & 1.6 & 1.6 & 1934 & 2.4 & 2.3 & $O C$ & 48 \\
\hline OC & 49 & 1274 & 90.4 & 4.5 & 2.0 & 3.1 & 1342 & 2.8 & 2.3 & OC & 49 \\
\hline$O C$ & 50 & 1337 & 95.4 & 2.5 & 0.9 & 1.3 & 1383 & 1.7 & 1.6 & $B C$ & 50 \\
\hline$D C$ & 51 & 1277 & 83.9 & 3.5 & 3.7 & 8.8 & 1330 & 2.2 & 1.8 & OC & 51 \\
\hline$D C$ & 52 & 972 & 92.5 & 3.4 & 2.0 & 2.2 & 1004 & 2.0 & 1.2 & $O C$ & 52 \\
\hline$O C$ & 53 & 2970 & 78.8 & 3.2 & 5.1 & 12.9 & 3137 & 2.8 & 2.5 & OC & 53 \\
\hline$O C$ & 54 & $123 i$ & 90.2 & 2.3 & 1.3 & 6.1 & 1296 & 2.5 & 2.2 & OC & 54 \\
\hline$O C$ & 55 & 1285 & 92.5 & 3.0 & 1.5 & 3.1 & 1282 & 1.9 & 1.8 & $O C$ & 55 \\
\hline$O C$ & 56 & 1245 & 93.8 & 1.2 & 2.6 & 2.4 & 1269 & 0.9 & 0.9 & $O C$ & 56 \\
\hline$O C$ & 57 & 1297 & 93.2 & 3.0 & 1.2 & 2.6 & 1343 & 1.7 & 1.7 & oC & 57 \\
\hline$O C$ & 58 & $16: 2$ & 94.4 & 2.7 & 1.1 & 1.8 & 1673 & 1.7 & 2.0 & $D C$ & 58 \\
\hline oc & 59 & 1170 & 92.0 & 2.8 & 1.1 & 4.0 & 1539 & 1.6 & 1.6 & DC & 59 \\
\hline$D C$ & 60 & 138 & 94.0 & 3.3 & 1.3 & 1.5 & 1856 & 2.0 & 1.7 & DC & 60 \\
\hline$D C$ & 61 & 1306 & 98.9 & 0.6 & 0.3 & 0.2 & 13207 & 0.4 & 0.4 & OC & 61 \\
\hline$O C$ & 62 & 1007 & 92.0 & 4.2 & 1.9 & 1.9 & 1904 & 2.5 & 2.6 & OC & 62 \\
\hline OC & 63 & $\because 45$ & 98.4 & 0.6 & 0.4 & 0.7 & 2458 & 0.5 & 0.0 & $O C$ & 63 \\
\hline OC & 64 & $: 715$ & 88.5 & 3.9 & 1.7 & 5.9 & 1806 & 2.4 & 2.7 & OC & 64 \\
\hline OC & 85 & 472 & 89.1 & 4.4 & 1.8 & 4.7 & 1577 & 4.1 & 2.5 & $\mathrm{OC}$ & 65 \\
\hline DC & 66 & 1571 & 91.1 & 3.8 & 1.4 & 3.8 & 1641 & 2.0 & 2.3 & OC & 66 \\
\hline$O C$ & 67 & 1270 & 94.8 & 2.7 & 1.0 & 1.5 & 1308 & 1.4 & 1.5 & OC & 67 \\
\hline
\end{tabular}




\section{$\underline{\text { Railroad Depot }}$}

The 0ld Ellenton Railroad Depot is located near the intersection of Railroad Avenue and Main Street (SRP Rt. 3), as shown on the Index Map in Figure 1. A 20 site grid on $10 \times 20$ foot centers was sampled for soil gas and analyzed for $\mathrm{C}_{1}-\mathrm{C}_{4}$ hydrocarbons. These site locations are shown on Figure 15. The data is shown in Figures 16, 17 and 18, and tabulated on Tables 9 and 10 . As shown in Figure 16, and documented in Table 9, no significant methane anomalies were found. One site was found to be somewhat anomalous in terms of percent butane (see Figure 18), however, as shown on Figure 17, it was only moderately significant in terms of butane magnitude, suggesting the level of contamination is small. No soil samples for gasoline range hydrocarbons were taken. 


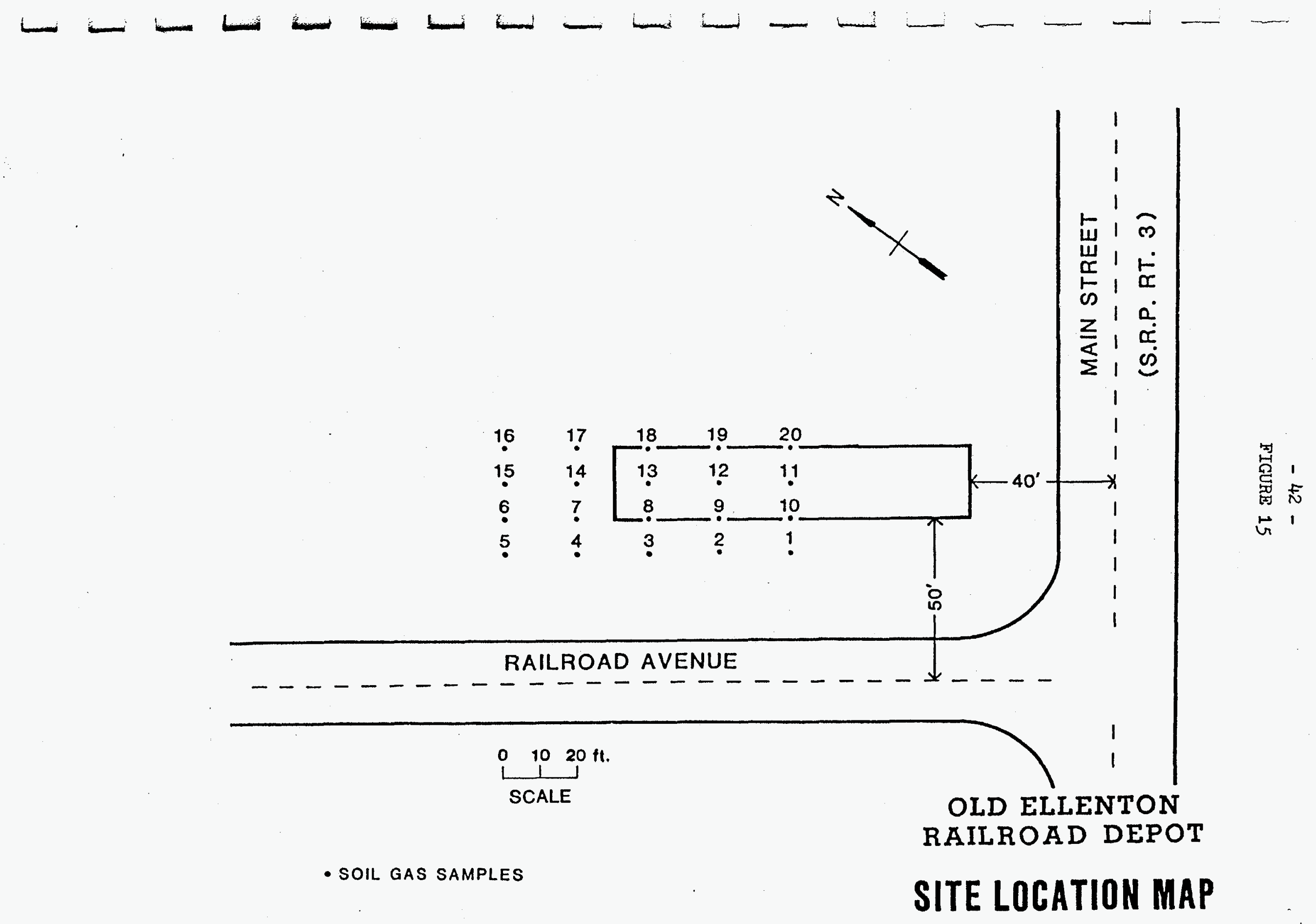





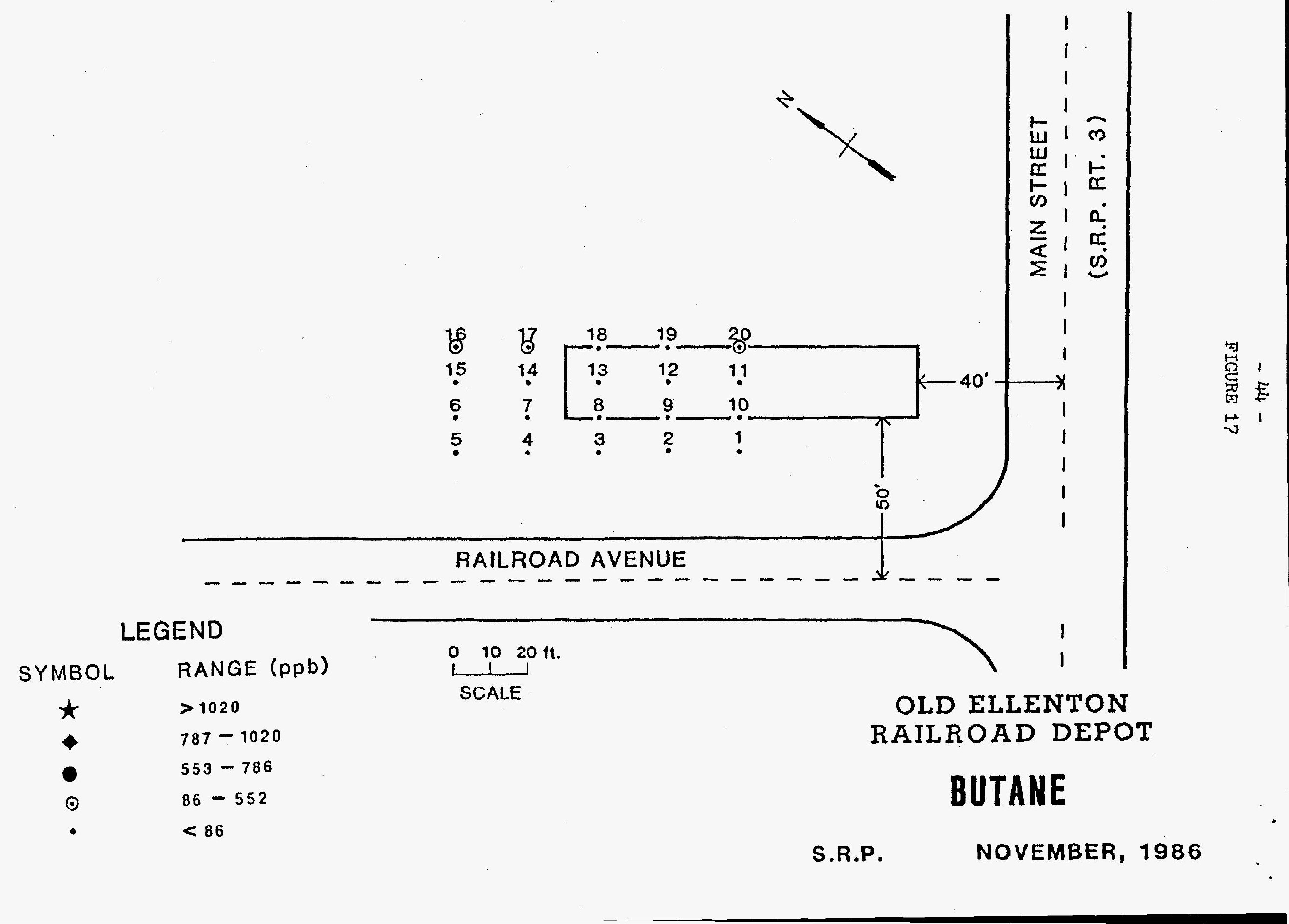




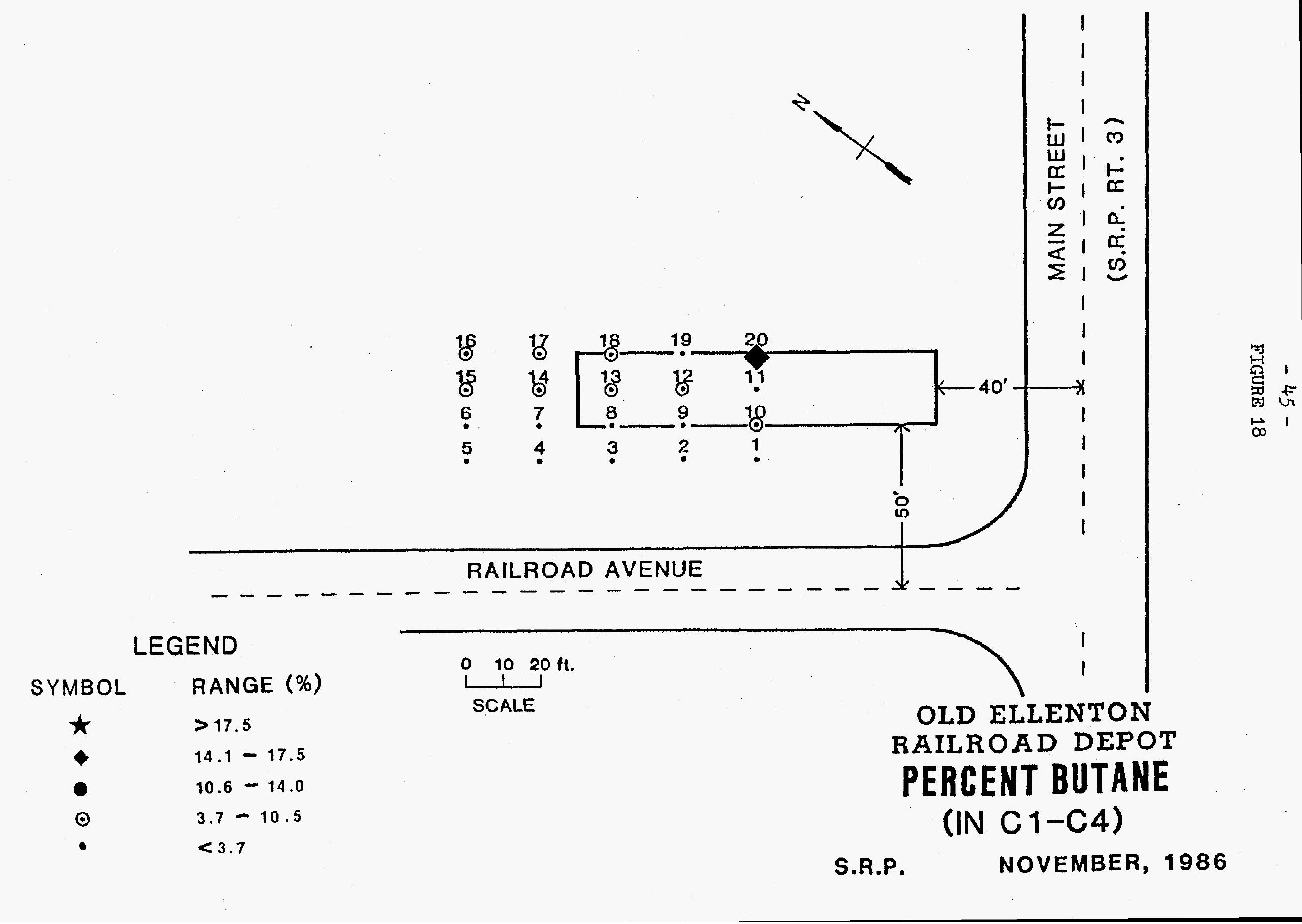


- E. I. DUPOKT DE NEHOURS CO. IKC., S.R.P., AIXEN, S.C. --.-

-- OLD ELLENTOY / R.R. DEPOT -- 3 FT. PROBE SURVEY, SEPT. 1986 --.-

--- SOIL gAS CONCENTRATIONS ----

\begin{tabular}{|c|c|c|c|c|c|c|c|c|c|c|}
\hline $\begin{array}{c}\text { SAMP } \\
\vdots\end{array}$ & & $\begin{array}{r}\text { METHANE } \\
\text { PPB }\end{array}$ & $\begin{array}{r}\text { ETHANE } \\
P P B\end{array}$ & $\begin{array}{r}\text { PROPANE } \\
\text { PPB }\end{array}$ & $\begin{array}{c}\text { 1-BUTANE } \\
\text { PPB }\end{array}$ & $\begin{array}{r}\text { W-BUTANE } \\
\text { PPB }\end{array}$ & $\begin{array}{r}\text { ETHYLENE } \\
\text { PPB }\end{array}$ & $\begin{array}{c}\text { PROPYLENE } \\
\text { PPB }\end{array}$ & SAMI & \\
\hline RD & 1 & 842 & 29 & 11 & 31 & - & 29 & 27 & RB & 1 \\
\hline$R D$ & 2 & 732 & 28 & 10 & 13 & - & 17 & 20 & $R D$ & 2 \\
\hline$R D$ & 3 & 853 & 27 & 10 & 21 & - & 16 & 18 & $R D$ & 3 \\
\hline RD & 4 & 839 & 29 & 10 & 15 & - & 15 & 14 & RD & 4 \\
\hline RD & 5 & 753 & 20 & 9 & - & - & - & - & RD & 5 \\
\hline RD & 6 & 1014 & 26 & 10 & 18 & - & 14 & 16. & RD & 6 \\
\hline$R D$ & 7 & 675 & 24 & 8 & 17 & - & 12 & 12 & RD & 7 \\
\hline $\mathrm{RD}$ & 8 & 510 & 13 & - & 14 & - & - & - & RD & 8 \\
\hline$R D$ & 9 & 1000 & 24 & 10 & 19 & - & 16 & 17 & $R D$ & 9 \\
\hline $\mathrm{RD}$ & 10 & 1020 & 28 & 46 & 45 & 33 & 17 & 18 & $\mathrm{RD}$ & 10 \\
\hline$R D$ & 11 & 1115 & 21 & 7 & 23 & - & 13 & 24 & $R D$ & 11 \\
\hline RD & 12 & 713 & 30 & 12 & 31 & - & 21 & 23 & $\mathrm{RD}$ & 12 \\
\hline$R D$ & 13 & 1663 & 56 & 25 & 76 & - & 42 & 35 & $R D$ & 13 \\
\hline$R D$ & 14 & 896 & 34 & 15 & 61 & - & 26 & 29 & $R D$ & 14 \\
\hline$R D$ & 15 & 817 & 30 & 12 & 47 & - & 17 & 19 & $R D$ & 15 \\
\hline$R D$ & 16 & 1999 & 73 & 35 & 119 & 14 & 39 & 41 & 80 & 16 \\
\hline$R D$ & 17 & 2792 & 114 & 42 & 139 & 14 & 156 & 113 & RD & 17 \\
\hline$R D$ & 18 & 868 & 25 & 10 & 36 & - & 12 & 15 & RD & 18 \\
\hline$R D$ & 19 & 750 & 17 & 8 & 18 & - & 13 & 12 & $R D$ & 19 \\
\hline RD & 20 & 926 & 60 & 62 & 190 & 14 & 22 & 26 & RD & 20 \\
\hline
\end{tabular}


--. E. 1. DUPONT DE MEROURS CO. INC., S.R.P., AIKEN, S.C. --

OLD ELLENTOY / R.R. DEPOT - 3 FT. PRDBE SURUEY, SEPT. 1986

-.-- SOIL GAS PERCENTAGES --.-

\begin{tabular}{|c|c|c|c|c|c|c|c|c|c|c|c|}
\hline SAMP & & $\begin{array}{l}\text { TOTAL } \\
\text { CI-CA }\end{array}$ & $\begin{array}{r}\text { METHANE } \\
\text { ICI }\end{array}$ & $\begin{array}{r}\text { ETHANE } \\
2 C 2\end{array}$ & $\begin{array}{r}\text { PROPANE } \\
\text { ICJ }\end{array}$ & $\begin{array}{l}\text { BUTAHE } \\
\text { ZCA }\end{array}$ & $\begin{array}{c}\text { TOTAL } \\
\text { HYDCARB }\end{array}$ & $\begin{array}{r}\text { ETHYLENE } \\
?\end{array}$ & $\begin{array}{c}\text { PYLENE } \\
z\end{array}$ & \multicolumn{2}{|c|}{$\begin{array}{c}\text { SAMPLE } \\
t\end{array}$} \\
\hline RD & 1 & 913 & 92.2 & 3.2 & 1.2 & 3.1 & 969 & 3.0 & 2.8 & $R D$ & \\
\hline RD & 2 & 783 & 93.5 & 3.6 & 1.3 & 1.7 & 820 & 2.1 & 2.1 & RD & 2 \\
\hline RD & 3 & 911 & 93.6 & 3.0 & 1.1 & 2.3 & 945 & 1.7 & 1.9 & RD & 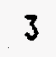 \\
\hline RD & 4 & 893 & 94.0 & 3.2 & 1.1 & 1.7 & 922 & 1.6 & 1.5 & RD & 1 \\
\hline RD & 5 & 782 & 96.3 & 2.6 & 1.2 & 0.0 & 782 & 0.0 & 0.0 & RD & \\
\hline RD & 6 & 1068 & 91.9 & 2.4 & 0.9 & 1.7 & 1098 & 1.3 & 1.5 & RD & \\
\hline$R D$ & 7 & 724 & 93.2 & 3.3 & 1.1 & 2.3 & 748 & 1.6 & 1.2 & AD & 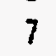 \\
\hline $\mathrm{RD}$ & 8 & 537 & 95.0 & 2.4 & 0.0 & 2.6 & 537 & 0.0 & 6.0 & RD & 0 \\
\hline RD & 9 & 1053 & 95.0 & 2.3 & 0.9 & 1.8 & 1086 & 1.5 & 1.6 & RD & $y$ \\
\hline RD & 10 & 1172 & 87.0 & 2.4 & 3.9 & 6.7 & 1207 & 1.4 & 1.5 & RD & 10 \\
\hline RD & 11 & 1166 & 95.6 & 1.8 & 0.6 & 2.0 & 1203 & 1.1 & $\therefore 0$ & RD & 11 \\
\hline $\mathrm{RD}$ & 12 & 789 & 90.1 & 3.8 & 1.5 & 4.3 & 833 & 2.5 & $\therefore 8$ & $R D$ & 12 \\
\hline$R D$ & 13 & 1820 & 91.1 & 3.1 & 1.4 & 4.2 & 1897 & 2.2 & .8 & RD & \\
\hline RD & 14 & 1006 & 89.1 & 3.1 & 1.5 & 6.1 & 1061 & 2.5 & 2.7 & $\mathrm{RD}$ & \\
\hline$R D$ & 15 & 906 & 90.2 & 3.3 & 1.3 & 5.2 & 942 & 1.8 & 2.0 & RD & \\
\hline $\mathrm{RD}$ & 16 & 2240 & 89.2 & 3.3 & 1.6 & 5.9 & 2320 & 1.7 & 1.8 & $R D$ & \\
\hline$R D$ & 17 & 3101 & 90.0 & 3.7 & 1.4 & 4.9 & 3370 & 4.6 & 3.4 & $R D$ & \\
\hline$R D$ & 18 & 939 & 92.1 & 2.7 & 1.1 & 3.8 & 966 & 1.2 & 1.6 & $R D$ & \\
\hline$R D$ & 19 & 793 & 94.6 & 2.1 & 1.0 & 2.3 & 818 & 1.6 & 1.5 & $R D$ & \\
\hline $\mathrm{RD}$ & 20 & 1252 & 74.0 & 4.8 & 5.0 & 16.3 & 1300 & 1.7 & 2.0 & RD & 2 \\
\hline
\end{tabular}


The Chevrolet Dealer

The 0ld Ellenton Chevrolet dealership was located on the south side of Main Street, as shown on the Index Map in Figure 1. Fourteen sites were sampled for soil gas. As shown in Figure 19, 9 sites were located parallel to the building foundation and 5 sites were located at an adjacent area previously used as an "oil can dump." These fourteen samples were analyzed for $C_{1}-C_{4}$ hydrocarbons. No anomalous levels of any hydrocarbons were found. The data is shown in Figures 20,21 and 22, and is tabulated in Tables 11 and 12. No soil samples were taken for gasoline range hydrocarbon analysis. 
FIGURE 19
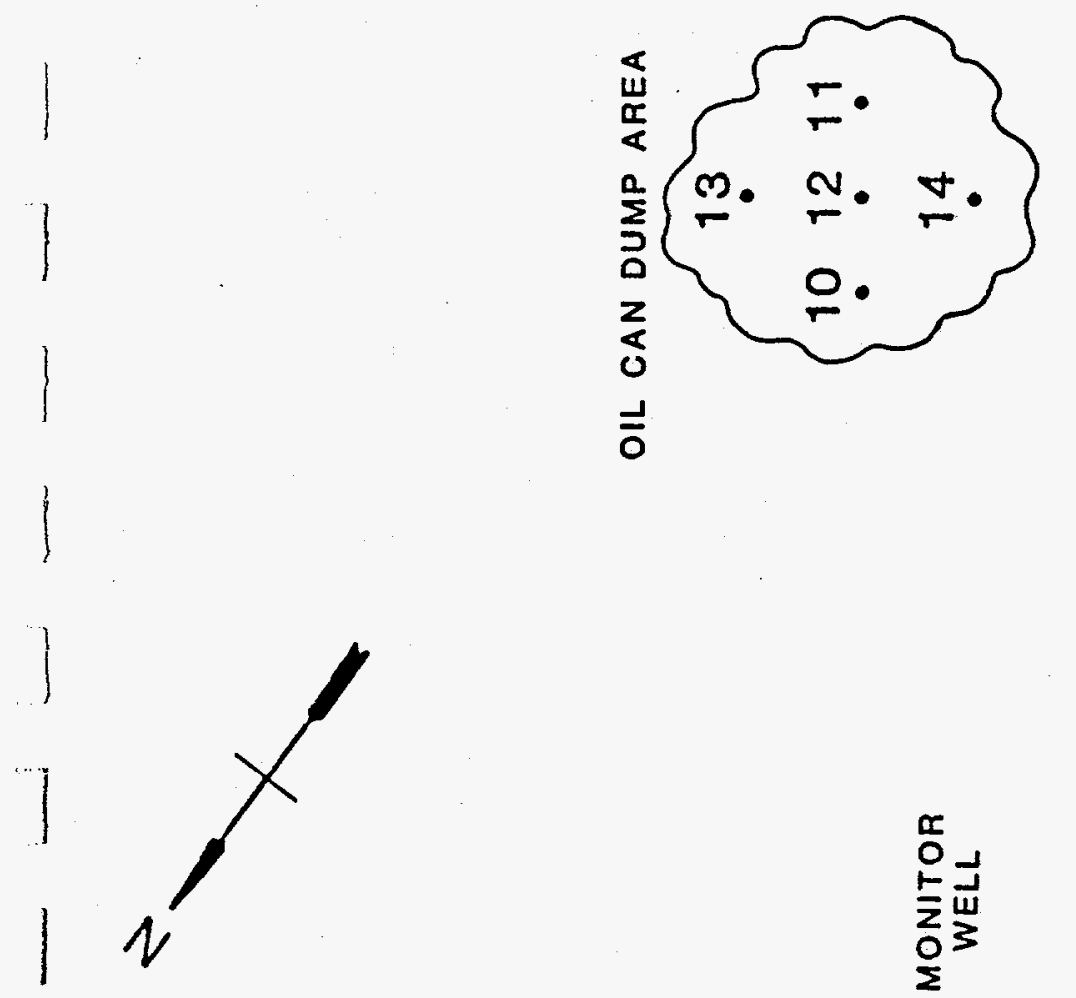

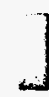

1

1

]

]

I

]

1

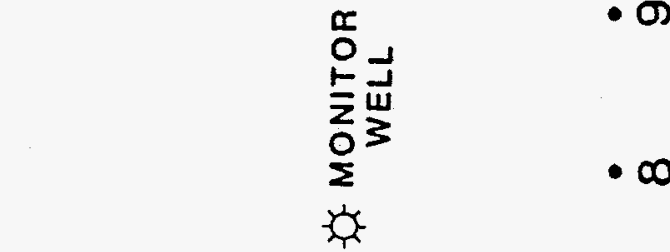

- $\sigma$

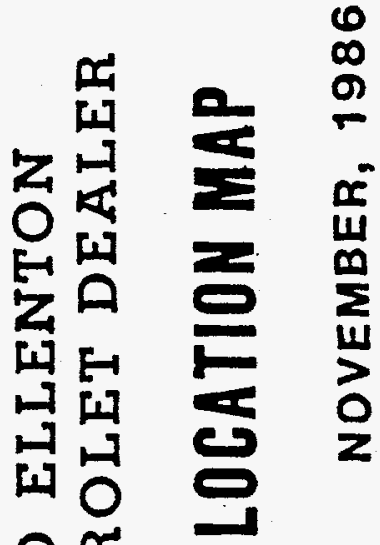

12

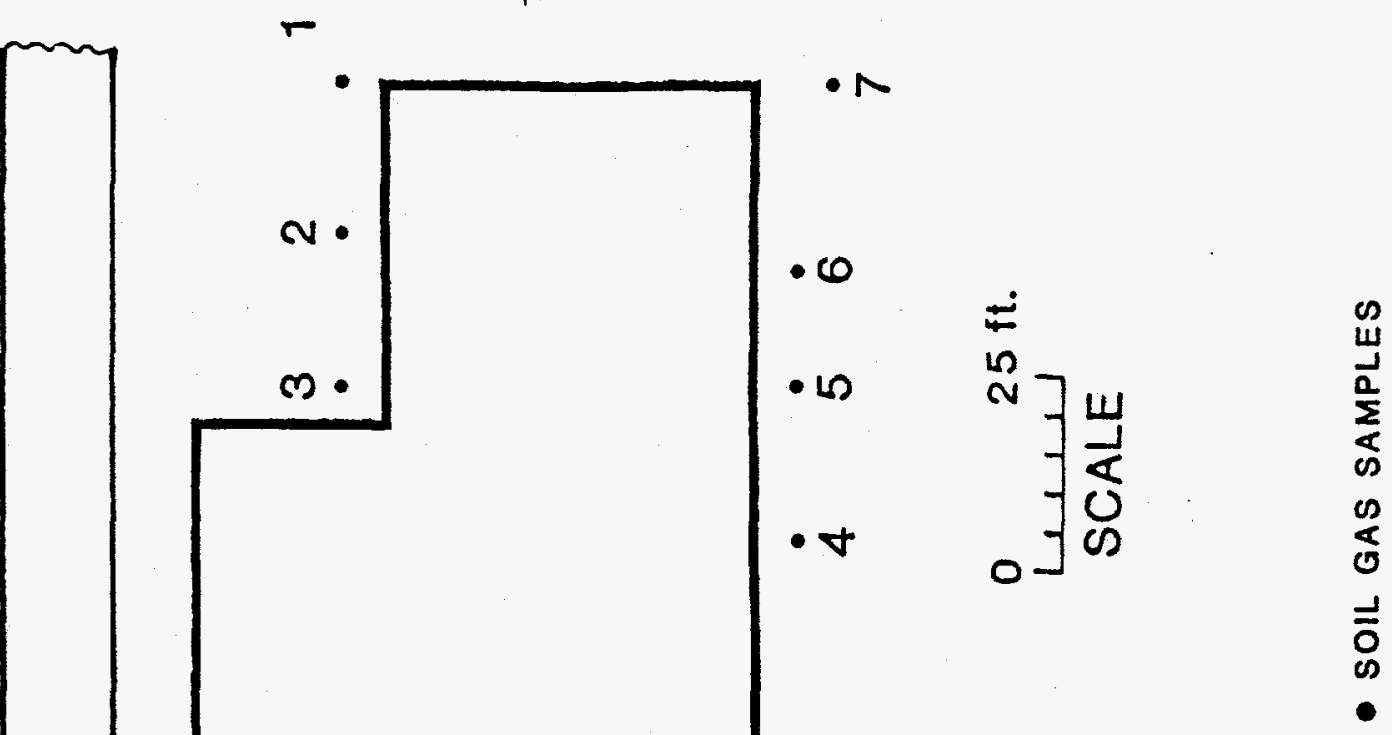

1 1

( $\left(\varepsilon \cdot 4 \cdot d^{\prime} \cdot y^{\circ} s\right)$

焉

$\frac{\dot{a}}{\dot{c}}$

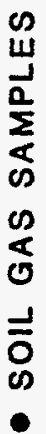

1

1 


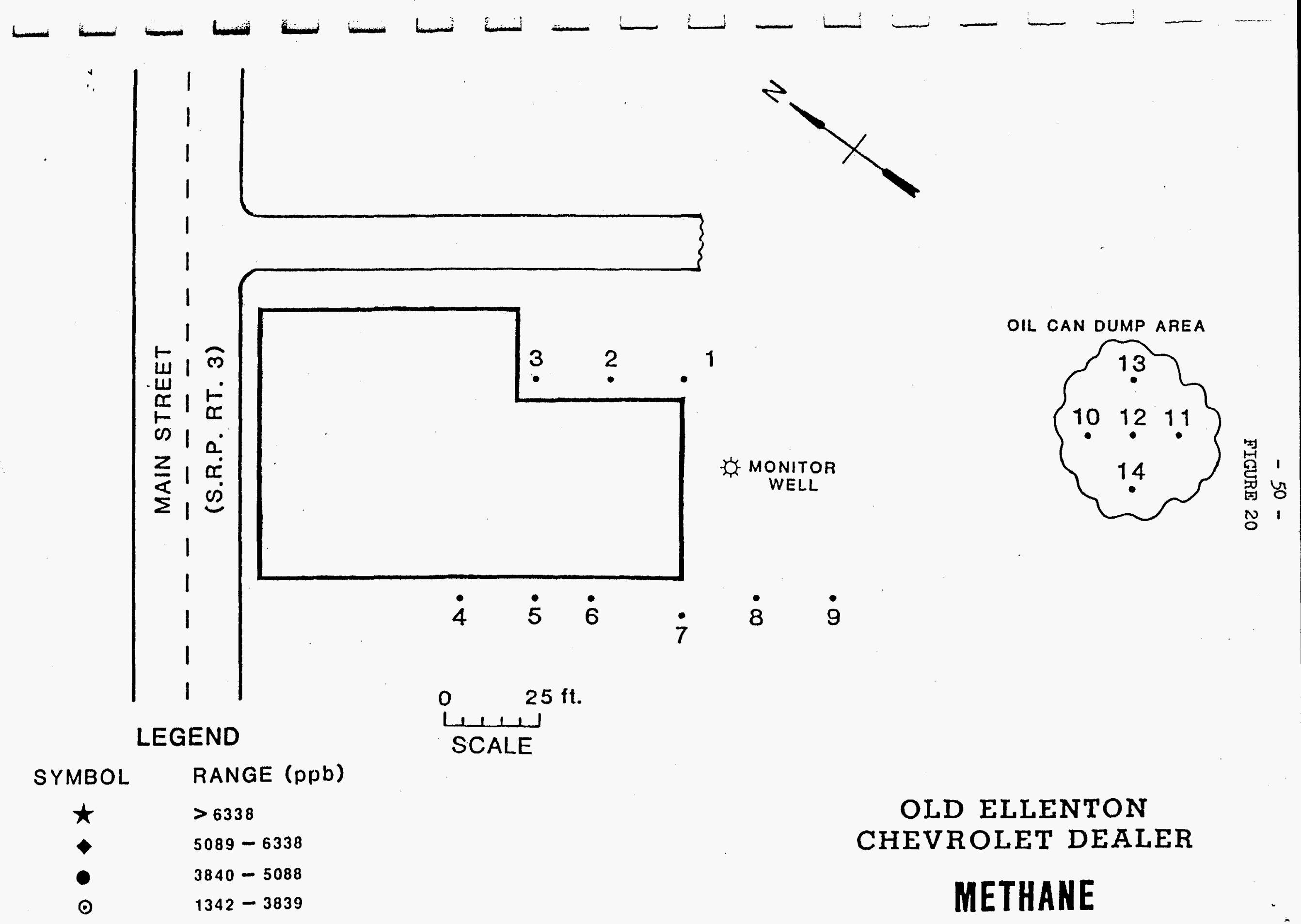





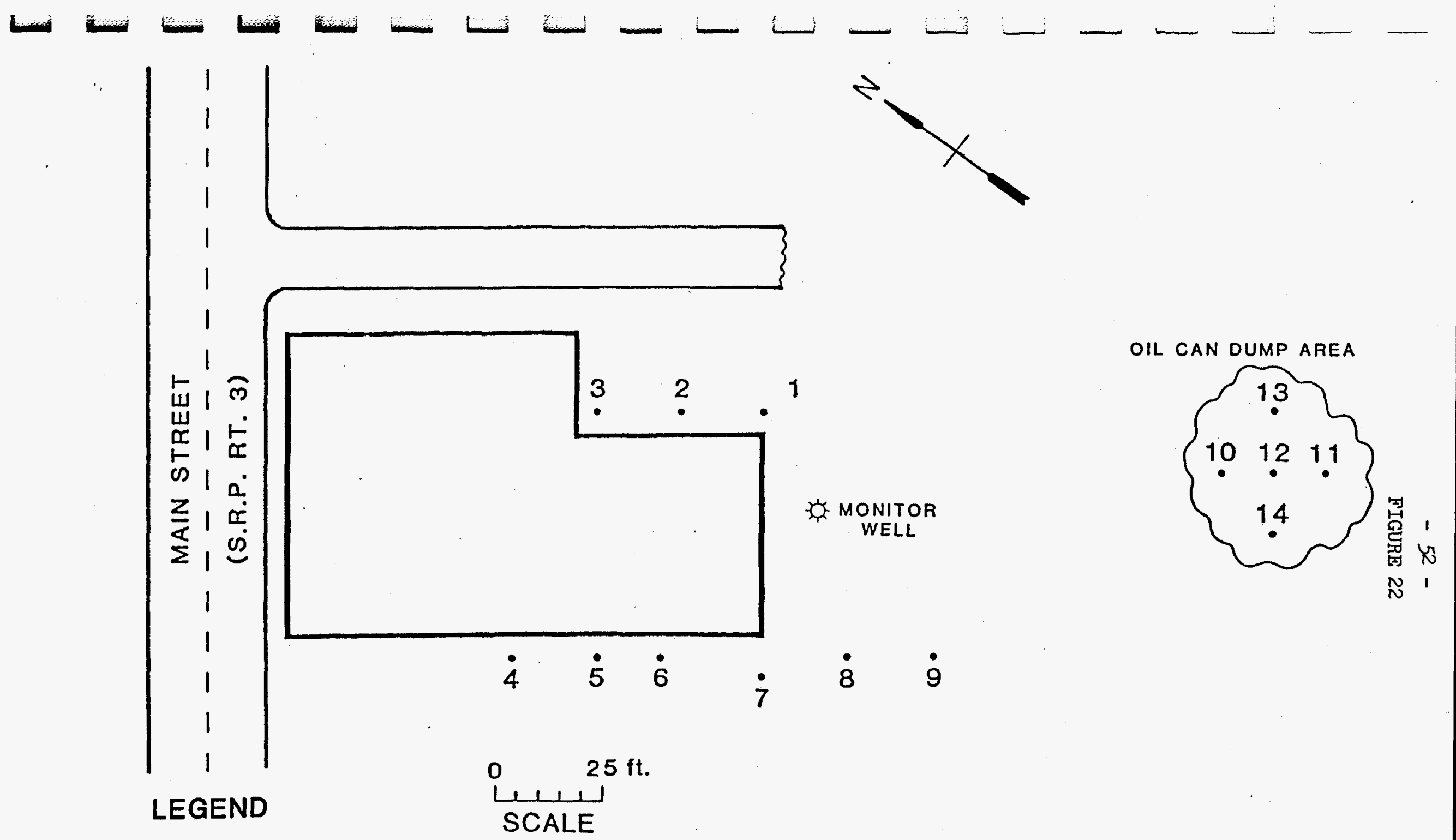

$\begin{array}{cl}\text { SYMBOL } & \text { RANGE (\%) } \\ \star & >17.5 \\ \bullet & 14.1-17.5 \\ \bullet & 10.6-14.0 \\ \odot & 3.7-10.5 \\ \bullet & <3.7\end{array}$

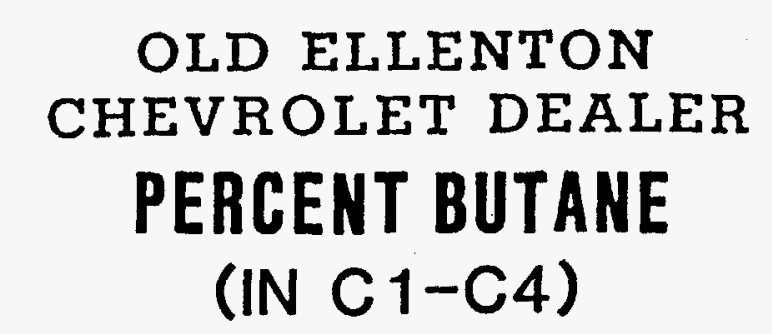

S.R.P.

NOVEMBER, 1986 
E. 1. DUPONT DE MEMOURS CO. INC., S.R.P., AIKEN, S.C. ---

OLD ELLENTON / CHEYY DEALER - 3 FT. PROBE SURUEY, SEPT. 1986 ----

-.-- SOIL GAS CONCERTRATIONS -.--

\begin{tabular}{|c|c|c|c|c|c|c|c|c|c|c|}
\hline \multirow{2}{*}{\multicolumn{2}{|c|}{$\begin{array}{c}\text { SANPLE } \\
\end{array}$}} & METHANE & ETHANE & PROPANE & & & ETHYLENE & PROPYLENE & \multirow{2}{*}{\multicolumn{2}{|c|}{ SAKPLE }} \\
\hline & & & PPB & PPB & PPB & PPB & PPB & PPB & & \\
\hline$C D$ & 1 & 732 & 25 & 8 & 11 & - & 19 & 18 & $C D$ & 1 \\
\hline$C D$ & 2 & 560 & 15 & 5 & - & - & 13 & 12 & $C D$ & 2 \\
\hline CD & 3 & 1047 & 38 & 16 & 27 & - & 40 & 40 & CD & 3 \\
\hline$C D$ & 4 & 923 & 29 & 10 & - & - & 16 & 15 & $C D$ & 4 \\
\hline CD & 5 & 514 & 24 & 9 & - & - & 15 & 16 & $C D$ & 5 \\
\hline CD & 6 & 726 & 26 & 10 & 12 & - & 15 & 15 & $C D$ & 6 \\
\hline$C D$ & 7 & 711 & 24 & - & - & - & 21 & - & $C D$ & 7 \\
\hline$C D$ & 8 & 783 & 56 & 24 & 21 & - & 35 & 39 & $\mathrm{CD}$ & 8 \\
\hline CD & 9 & 771 & 60 & 23 & - & - & 32 & 35 & CO & 9 \\
\hline$C D$ & 10 & 530 & 23 & - & - & - & 22 & - & CD & 10 \\
\hline CD & 11 & 609 & 15 & 5 & - & - & 15 & 11 & CD & 11 \\
\hline$C D$ & 12 & 491 & 15 & $b$ & - & - & 15 & - & $C D$ & 12 \\
\hline$C D$ & 13 & 528 & 22 & 11 & 12 & - & 20 & 20 & CD & 13 \\
\hline CD & 14 & 304 & 12 & 5 & - & - & 9 & - & $C D$ & 14 \\
\hline
\end{tabular}


--- E. I. DUPONT DE MEKOURS CO. INC., S.R.P., AIKEN, S.C. ----

--- OLD ELLENTOK / CHEVY DEALER -- 3 FT. PROBE SURUEY, SEPT. 1986 -..-

--- SOIL GAS PERCENTAGES

\begin{tabular}{|c|c|c|c|c|c|c|c|c|c|c|c|}
\hline \multicolumn{2}{|c|}{ SAHPLE } & \multirow{2}{*}{$\begin{array}{c}\text { TOTAL } \\
\text { C1-C4 } \\
776\end{array}$} & \multirow{2}{*}{$\begin{array}{r}\text { METHANE } \\
\text { ZCI } \\
94.3\end{array}$} & \multirow{2}{*}{$\begin{array}{r}\text { ETHANE } \\
2 C 2 \\
3.2\end{array}$} & \multirow{2}{*}{$\begin{array}{r}\text { PROPANE } \\
\text { IC3 } \\
1.0\end{array}$} & \multirow{2}{*}{$\begin{array}{r}\begin{array}{r}\text { BUTANE } \\
\text { ZC4 }\end{array} \\
1.4\end{array}$} & \multirow{2}{*}{$\begin{array}{c}\begin{array}{c}\text { TOTAL } \\
\text { HYDCARB }\end{array} \\
813\end{array}$} & \multicolumn{2}{|c|}{$\begin{array}{c}\text { ETHYLENE PROPYLENE } \\
2\end{array}$} & \multicolumn{2}{|c|}{ SAKPLE } \\
\hline$C D$ & 1 & & & & & & & 2.3 & 2.2 & $C D$ & 1 \\
\hline CD & 2 & 580 & 96.6 & 2.6 & 0.9 & 0.0 & 605 & 2.1 & 2.0 & $C D$ & 2 \\
\hline$C D$ & 3 & 1128 & 92.8 & 3.4 & 1.4 & 2.4 & 1208 & 3.3 & 3.3 & $C D$ & 3 \\
\hline$C D$ & 4 & 962 & 95.9 & 3.0 & 1.0 & 0.0 & 993 & 1.6 & 1.5 & $C D$ & 4 \\
\hline$C D$ & 5 & 547 & 94.0 & 4.4 & 1.6 & 0.0 & 578 & 2.6 & 2.8 & $\mathrm{CD}$ & 5 \\
\hline$C D$ & 6 & 774 & 93.8 & 3.4 & 1.3 & 1.6 & 804 & 1.9 & 1.9 & $C D$ & 6 \\
\hline CD & 7 & 735 & 96.7 & 3.3 & 0.0 & 0.0 & 756 & 2.8 & 0.0 & $C D$ & 7 \\
\hline $\mathrm{CD}$ & 8 & 884 & 88.6 & 6.3 & 2.7 & 2.4 & 958 & 3.7 & 4.1 & $C D$ & 8 \\
\hline$C D$ & 9 & 854 & 90.3 & 7.0 & 2.7 & 0.0 & 921 & 3.5 & 3.8 & $C O$ & 9 \\
\hline$C D$ & 10 & 553 & 95.8 & 4.2 & 0.0 & 0.0 & 575 & 3.8 & 0.0 & $C D$ & 10 \\
\hline$C D$ & 11 & 629 & 96.8 & 2.4 & 0.8 & 0.0 & 655 & 2.3 & 1.7 & $\mathrm{CD}$ & 11 \\
\hline$C D$ & 12 & 515 & 95.9 & 2.9 & 1.2 & 0.0 & 530 & 2.8 & 0.0 & CD & 12 \\
\hline$C D$ & 13 & 573 & 92.1 & 3.8 & 1.9 & 2.1 & 613 & 3.3 & 3.3 & $C D$ & 13 \\
\hline $\mathrm{CD}$ & 14 & 321 & 94.7 & 3.7 & 1.6 & 0.0 & 330 & 2.7 & 0.0 & $C D$ & 14 \\
\hline
\end{tabular}


The School

The 01d Ellenton School was located along Russell Street, as shown on the Index Map in Figure 1. 45 sites were sampled for soil gas around the perimeter of the former schoolhouse. Site locations are shown on Figure 23. The data is shown in Figures 24, 25 and 26, and is presented in Tables 13 and 14. No anomalous levels were found. No soil samples for gasoline range hydrocarbons were taken at this location. 


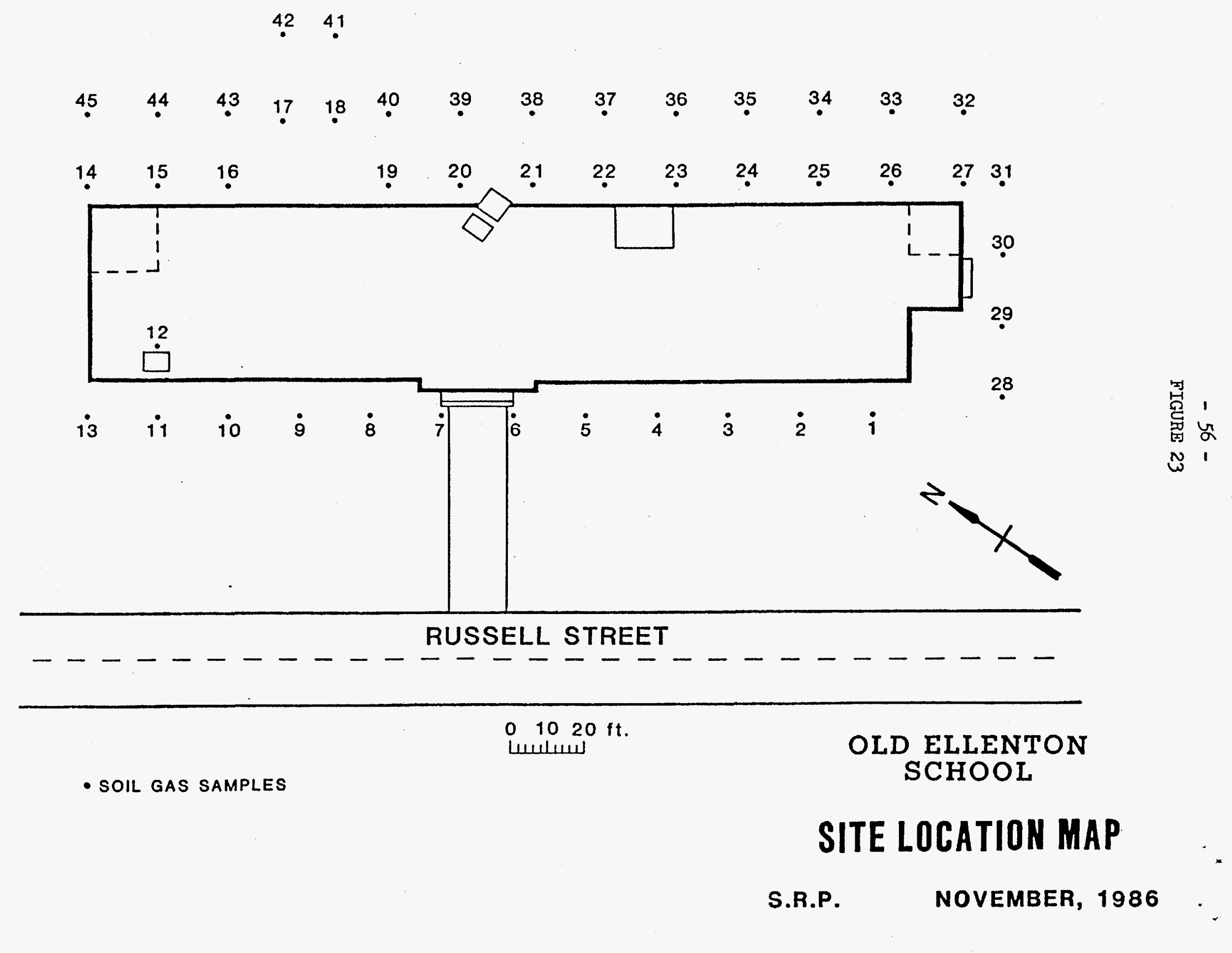




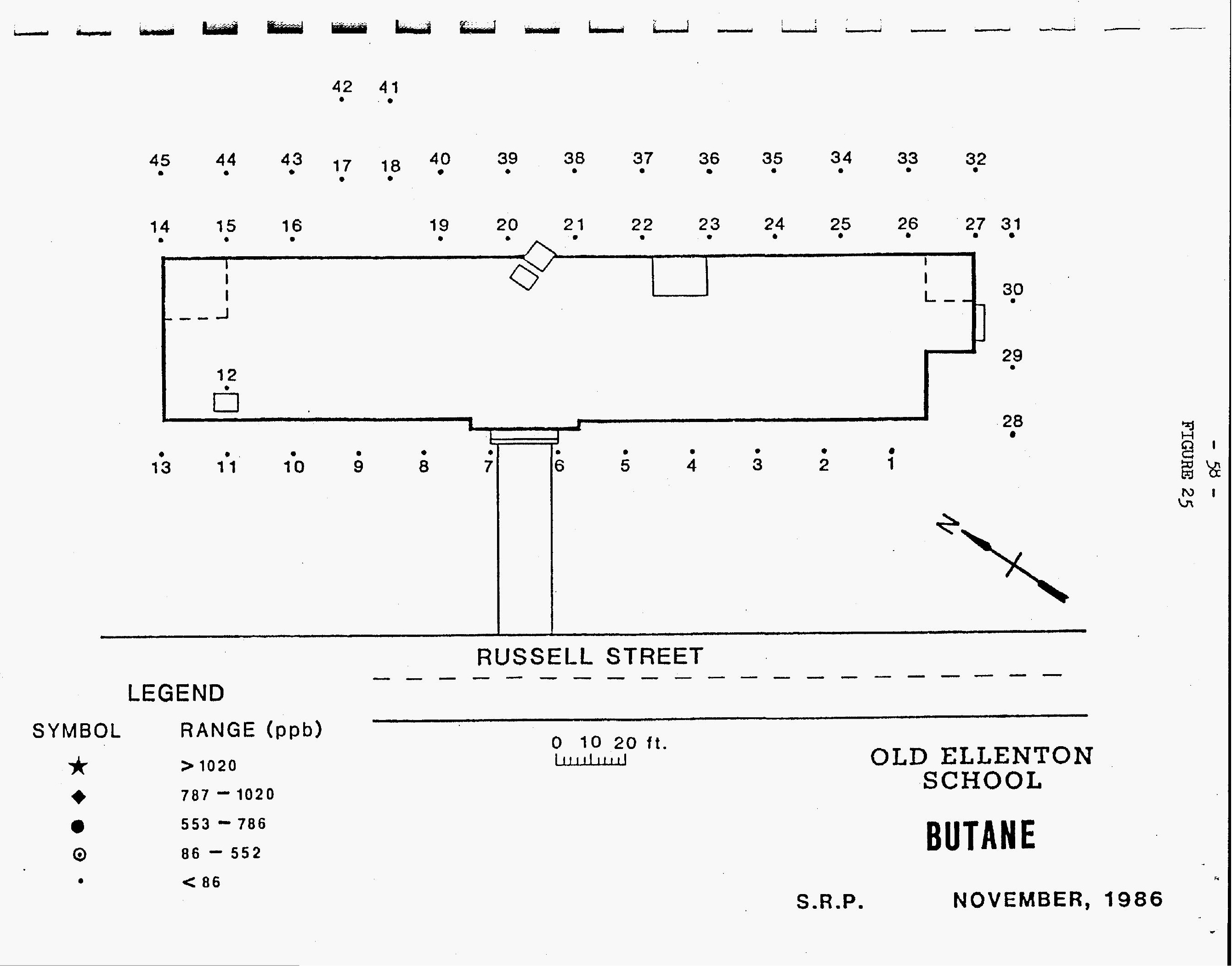
$42 \quad 41$

$\begin{array}{llllllllllllll}45 & 44 & 43 & 17 & 18 & 40 & 39 & 38 & 37 & 36 & 35 & 34 & 33 & 32\end{array}$
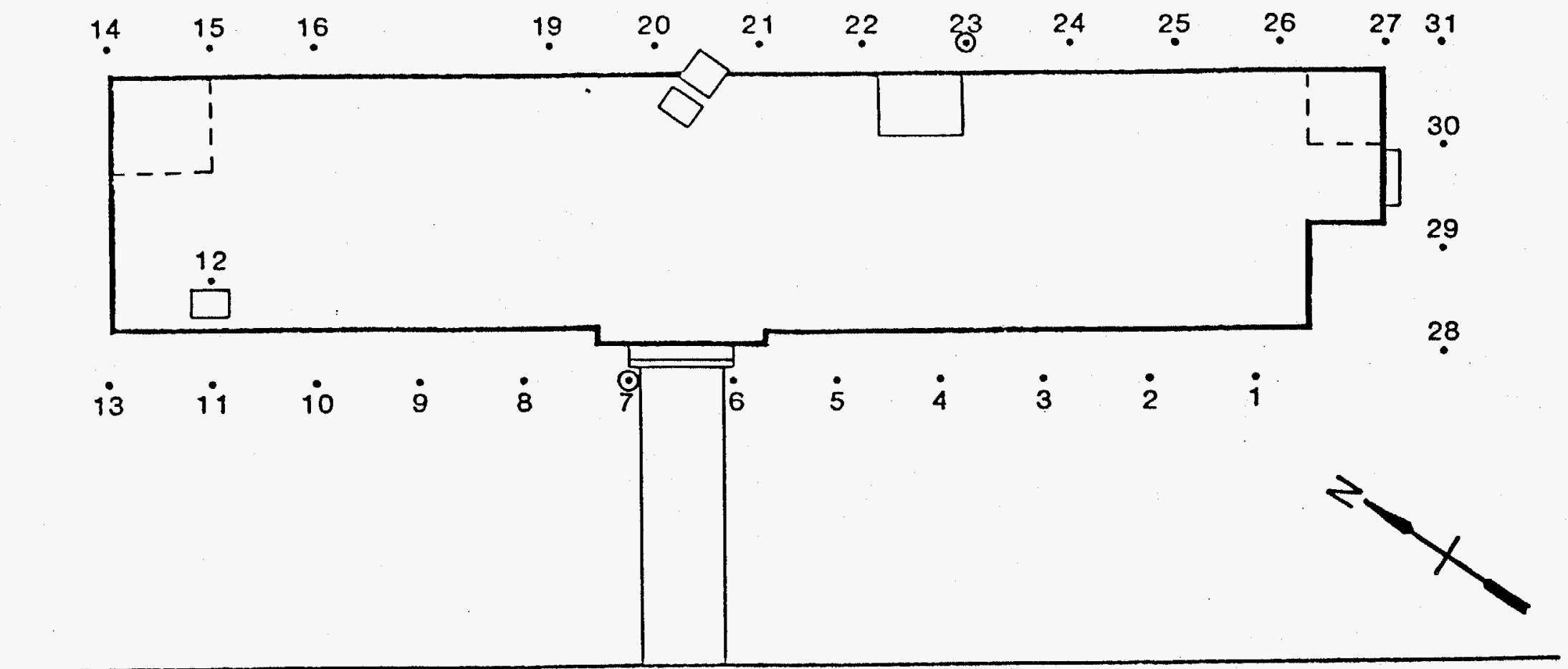

RUSSELL STREET

\section{LEGEND}

\section{SYMBOL RANGE (\%)}

$\begin{array}{ll}\star & >17.5 \\ \bullet & 14.1-17.5 \\ \bullet & 10.6-14.0 \\ \odot & 3.7-10.5 \\ \bullet & <3.7\end{array}$

$0 \quad 1020 \mathrm{ft}$.

سuluew
OLD ELLENTON SCHOOL PERCENT BUTANE

(IN C 1-C4) 
E. I. DUPONT DE MEMOURS CO. INC., S.R.P., AIKEN, S.C. ---

OLD ELLENTON / SCHOOL HOUSE -- 3 FT. PROBE SURVEY, SEPT. 1986 --.-.-- SOIL GAS CONCENTRATIOHS -.--

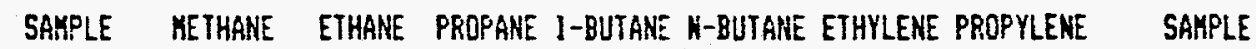

\begin{tabular}{|c|c|c|c|c|c|c|c|c|c|c|}
\hline \multicolumn{2}{|c|}{1} & PPB & FPE & PPE & PPE & PPE & PPE & PPB & & 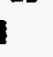 \\
\hline SH & 1 & 430 & 9 & - & - & - & 9 & - & SH & 1 \\
\hline SH & 2 & 521 & 15 & 8 & - & - & 9 & - & SH & 2 \\
\hline SH & 3 & 417 & 10 & - & - & - & 9 & - & SH & 3 \\
\hline SH & 4 & 406 & 8 & - & - & - & 8 & - & SH & 4 \\
\hline SH & 5 & 490 & 31 & 14 & 13 & - & 20 & 22 & SH & 5 \\
\hline SH & $b$ & 532 & 20 & 10 & - & - & 11 & - & SH & 6 \\
\hline SH & 7 & 539 & 17 & $B$ & 49 & - & .12 & - & 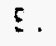 & 7 \\
\hline SH & 8 & 519 & 10 & 5 & - & - & 8 & - & $i h$ & 8 \\
\hline SH & 9 & 1541 & - & - & - & - & 6 & - & : H & 9 \\
\hline SH & 10 & 434 & 13 & 6 & - & - & 10 & - & : & 10 \\
\hline SH & 11 & 464 & 17 & 7 & - & - & 10 & - & $5 \mathrm{H}$ & 11 \\
\hline SH & 12 & 565 & 8 & - & - & - & 8 & - & SH & 12 \\
\hline SH & 13 & 587 & 29 & 12 & 15 & - & 17 & 16 & SH & 13 \\
\hline SH & 14 & 469 & 10 & 5 & 14 & - & 10 & - & SH & 14 \\
\hline SH & 15 & 527 & 13 & 6 & - & - & 10 & - & SH & 15 \\
\hline SH & 16 & 662 & 15 & 8 & 15 & - & 10 & - & SH & 16 \\
\hline SH & 17 & 1016 & 72 & 25 & 29 & - & $13 t$ & 103 & SH & 17 \\
\hline SH & 18 & 568 & 15 & 7 & - & - & 8 & - & SH & 18 \\
\hline SH & 19 & 672 & 14 & 6 & - & - & 12 & - & $5 H$ & 19 \\
\hline SH & 20 & 529 & 10 & - & - & - & 8 & - & SH & 20 \\
\hline SH & 21 & 489 & 16 & 7 & - & - & 12 & - & SH & 21 \\
\hline SH & 22 & 758 & 35 & 12 & 29 & - & 22 & 21 & SH & 22 \\
\hline $5 H$ & 23 & 823 & 43 & 16 & 46 & - & 24 & 25 & SH & 23 \\
\hline SH & 24 & 651 & $3 !$ & 17 & 25 & - & 15 & 17 & SH & 24 \\
\hline SH & 25 & 744 & 42 & 15 & 28 & - & 23 & 27 & SH & 25 \\
\hline$S H$ & 26 & 615 & 22 & $E$ & 15 & - & 9 & 15 & SH & $2 t$ \\
\hline SH & $2 i$ & 472 & 18 & 8 & 10 & - & 11 & - & SH & 27 \\
\hline SH & 28 & 316 & 7 & - & - & - & $\theta$ & - & SH & 28 \\
\hline SH & 29 & 291 & 7 & - & - & - & 7 & - & SH & 29 \\
\hline SH & 36 & 1147 & 35 & 11 & 19 & - & 17 & 15 & SH & 30 \\
\hline SH & 31 & 611 & 24. & 10 & 15 & - & 16 & 17 & SH & 31 \\
\hline SH & 32 & 363 & 14 & 6 & - & - & 9 & - & SH & 32 \\
\hline SH & 33 & 522 & 15 & - & - & - & 10 & - & SH & 33 \\
\hline SH & 34 & 552 & 19 & 11 & - & - & 11 & - & SH & 34 \\
\hline SH & 35 & $38 \dot{5}$ & 15 & 8 & - & - & 10 & - & SH & 35 \\
\hline SH & 36 & 361 & 15 & 7 & - & - & 12 & - & SH & 30 \\
\hline SH & 37 & 553 & 26 & 10 & 18 & - & 22 & 22 & SH & 37 \\
\hline SH & 38 & 570 & 23 & 8 & 14 & - & 14 & 16 & SH & 38 \\
\hline SH & 39 & 439 & 15 & - & - & - & 10 & - & SH & 39 \\
\hline SH & $4 \hat{v}$ & 702 & 33 & 12 & $2 t$ & - & 26 & 25 & SH & 40 \\
\hline SH & 41 & 778 & 27 & 9 & 23 & - & 19 & 19 & SH & 41 \\
\hline SH & 42 & 713 & 25 & 10 & 17 & - & 23 & 20 & SH & 42 \\
\hline SH & 13 & 633 & 25 & 10 & - & - & 14 & 14 & SH & 13 \\
\hline SH & 4 & 502 & 17 & - & - & - & - & - & SH & 14 \\
\hline SH & 45 & 508 & - & 8 & - & - & 14 & - & SH & 45 \\
\hline
\end{tabular}


-..- E. 1. DUPQNT DE MEMOURS CO. INC., S.R.P., AIKEN, S.C. -...

--- OLD ELLENTON / SCHOOL HOUSE -- 3 FT. PROBE SURUEY, SEPT., 1986

-..- SOIL GAS PERCENTAGES -...-

\begin{tabular}{|c|c|c|c|c|c|c|c|c|c|c|c|}
\hline SAMP & & $\begin{array}{l}\text { TOTAL } \\
\text { CI-CA }\end{array}$ & $\begin{array}{r}\text { METHANE } \\
\text { ZC: }\end{array}$ & $\begin{array}{r}\text { ETHANE } \\
\text { ZS: }\end{array}$ & $\begin{array}{r}\text { PROPAME } \\
\text { ZCS }\end{array}$ & $\begin{array}{l}\text { BUTANE } \\
\text { 2CA }\end{array}$ & $\begin{array}{c}\text { TOTAL } \\
\text { HYDCARE }\end{array}$ & $\begin{array}{c}\text { ETHYLENE } \\
\%\end{array}$ & $\begin{array}{c}\text { YLENE } \\
q\end{array}$ & $\begin{array}{r}\text { SAMPL } \\
1\end{array}$ & \\
\hline$S H$ & 1 & 439 & 97.9 & 2.1 & 0.0 & 0.0 & 448 & 2.6 & 0.0 & SH & , \\
\hline $5 H$ & 2 & 544 & 95.8 & 2.8 & 1.5 & 0.0 & 553 & 1.6 & 0.0 & SH & 2 \\
\hline SH & 3 & 427 & 97.7 & 2.3 & 0.0 & 0.0 & 436 & 2.1 & 0.0 & SH & 3 \\
\hline SH & 4 & 414 & 98.1 & 1.9 & 0.0 & 0.0 & 422 & 1.9 & 0.0 & SH & 4 \\
\hline SH & 5 & 548 & 89.1 & 5.7 & 2.6 & 2.4 & 590 & 3.4 & 3.7 & SH & 5 \\
\hline SH & $t$ & 562 & 94.7 & 3.6 & 1.8 & 0.0 & 573 & 1.9 & 0.0 & SH & $t$ \\
\hline SH & 7 & 613 & 87.9 & 2.8 & 1.3 & 8.0 & 625 & 1.9 & 0.0 & SH & 7 \\
\hline $\mathrm{SH}$ & 8 & 534 & 97.2 & 1.9 & 0.9 & 0.0 & 542 & 1.5 & 0.0 & SH & 8 \\
\hline SH & 9 & 1541 & 160.0 & 0.0 & 0.0 & 0.0 & 1547 & 0.4 & 0.0 & SH & 9 \\
\hline SH & 10 & 453. & 95.8 & 2.9 & 1.3 & 0.0 & 463 & 2.2 & 0.0 & SH & 10 \\
\hline SH & 11 & $488^{\circ}$ & 95.1 & 3.5 & 1.4 & 0.0 & 498 & 2.0 & 0.0 & SH & 11 \\
\hline SH & 12 & 573 & 98.6 & 1.4 & 0.0 & 0.0 & 581 & 1.4 & 0.0 & SH & 12 \\
\hline SH & 13 & 643 & 91.3 & 4.5 & 1.9 & 2.3 & $67 t$ & 2.5 & 2.4 & SH & 13 \\
\hline SH & 14 & 498 & 94.2 & 2.0 & 1.0 & 2.8 & 508 & 2.0 & 0.0 & SH & 14 \\
\hline $5 H$ & 15 & 546 & 96.5 & 2.4 & 1.1 & 0.0 & $55 t$ & 1.8 & 0.0 & SH & 15 \\
\hline SH & 15 & 700 & 94.6 & 2.1 & 1.1 & 2.1 & 710 & 1.4 & 0.0 & SH & 16 \\
\hline SH & 17 & 1142 & 89.0 & 6.3 & 2.2 & 2.5 & $138 \mathrm{i}$ & 9.8 & 7.5 & SH & 17 \\
\hline SH & 18 & 590 & 96.3 & 2.5 & 1.2 & 0.0 & 598 & 1.3 & 0.0 & SH & 18 \\
\hline SH & 19 & 692 & 97.1 & 2.0 & 0.9 & 0.0 & 704 & 1.7 & 0.0 & SH & 19 \\
\hline SH & 20 & 539 & 98.1 & 1.9 & 0.0 & 0.0 & 547 & 1.5 & 0.0 & SH & $2 i$ \\
\hline SH & 21 & 512 & 95.5 & 3.1 & 1.4 & 0.0 & 524 & 2.3 & 0.0 & SH & \\
\hline SH & 22 & 832 & 91.1 & 4.0 & 1.4 & 3.5 & 875 & 2.5 & 2.4 & SH & 22 \\
\hline SH & 23 & 928 & 88.7 & 4.6 & 1.7 & 5.0 & 977 & 2.5 & 2.6 & $\mathrm{SH}$ & 23 \\
\hline SH & 24 & 724 & 89.9 & 4.3 & 2.3 & 3.5 & $75 \mathrm{~s}$ & 2.0 & 2.2 & SH & 24 \\
\hline $5 H$ & 25 & 829 & 89.7 & 5.1 & 1.6 & 3.4 & 875 & 2.6 & 3.1 & $5 \mathrm{H}$ & 2 \\
\hline SH & 26 & 665 & 93.2 & 3.3 & 1.2 & 2.3 & $68:$ & 1.3 & 2.2 & SH & \\
\hline SH & 27 & 514 & 91.8 & 3.5 & 1.6 & 3.1 & $522^{2}$ & 2.1 & 0.6 & SH & \\
\hline SH & 28 & 323 & 97.8 & 2.2 & 0.0 & 0.0 & 331 & 2.4 & 0.0 & SH & \\
\hline SH & 29 & 298 & 97.7 & 2.3 & 0.0 & 0.0 & 305 & 2.3 & 0.0 & SH & \\
\hline SH & 30 & 1212 & 94.6 & 2.9 & 0.9 & 1.6 & 1244 & 1.4 & 1.2 & SH & \\
\hline SH & 31 & 660 & 92.6 & 3.6 & 1.5 & 2.3 & 693 & 2.3 & 2.5 & $\mathrm{SH}$ & \\
\hline SH & 32 & 383 & 94.8 & 3.7 & 1.6 & 0.0 & 392 & 2.3 & 0.0 & SH & \\
\hline SH & 33 & 537 & 97.2 & 2.8 & 0.0 & 0.0 & 547 & 1.8 & 0.0 & SH & \\
\hline SH & 34 & 582 & 94.8 & 3.3 & 1.9 & 0.0 & 593 & 3.9 & 0.0 & $\mathrm{SH}$ & \\
\hline $\mathrm{SH}$ & 35 & 409 & 94.4 & 3.7 & 2.0 & 0.0 & 419 & 2.1 & 0.0 & $\mathrm{SH}$ & \\
\hline SH & 36 & 383 & 94.3 & 3.9 & 1.8 & 0.0 & 395 & 3.0 & 0.0 & $5 H$ & \\
\hline $\mathrm{SH}$ & 37 & 607 & 91.1 & 4.3 & 1.6 & 3.0 & 651 & 3.4 & 3.4 & $\mathrm{SH}$ & \\
\hline $5 H$ & 30 & 615 & 92.7 & 3.7 & 1.3 & 2.3 & 645 & 2.2 & 2.5 & $5 \mathrm{H}$ & \\
\hline SH & 39 & 154 & 96.7 & 3.3 & 0.0 & 0.0 & 164 & 2.2 & 0.0 & SH & \\
\hline SH & 40 & 773 & 90.8 & 4.3 & 1.6 & 3.4 & 824 & 3.2 & 3.0 & $\mathrm{SH}$ & \\
\hline SH & $4 !$ & $83 ?$ & 93.0 & 3.2 & 1.1 & 2.7 & 875 & 2.2 & 2.2 & SH & \\
\hline SH & 42 & 765 & 93.2 & 3.3 & 1.3 & 2.2 & 800 & 2.8 & 2.5 & $\mathrm{SH}$ & \\
\hline SH & 43 & 668 & 94.8 & 3.7 & 1.5 & 0.0 & 696 & 2.0 & 2.0 & SH & \\
\hline SH & 44 & 519 & 96.7 & 3.3 & 0.0 & 0.0 & 519 & 0.0 & 0.0 & SH & \\
\hline SH & 45 & 516 & 98.4 & 0.0 & 1.6 & 0.0 & 530 & 2.6 & 0.0 & SH & \\
\hline
\end{tabular}




\section{Dry Cleaner}

The 01d Ellenton dry cleaner was located near the intersection of Jackson and Center Streets. Soil samples from a 16 site grid on $40 \mathrm{ft}$ centers were taken from a depth of 18-24 inches and analyzed for nine halocarbons listed in Table 15. The site locations are shown in Figure 27. Particular interest was directed toward tetrachloroethylene which may have been used as a dry cleaning fluid. No halocarbons were found above the minimum detection levels as listed in Table 1. No other analyses were performed at this location. 


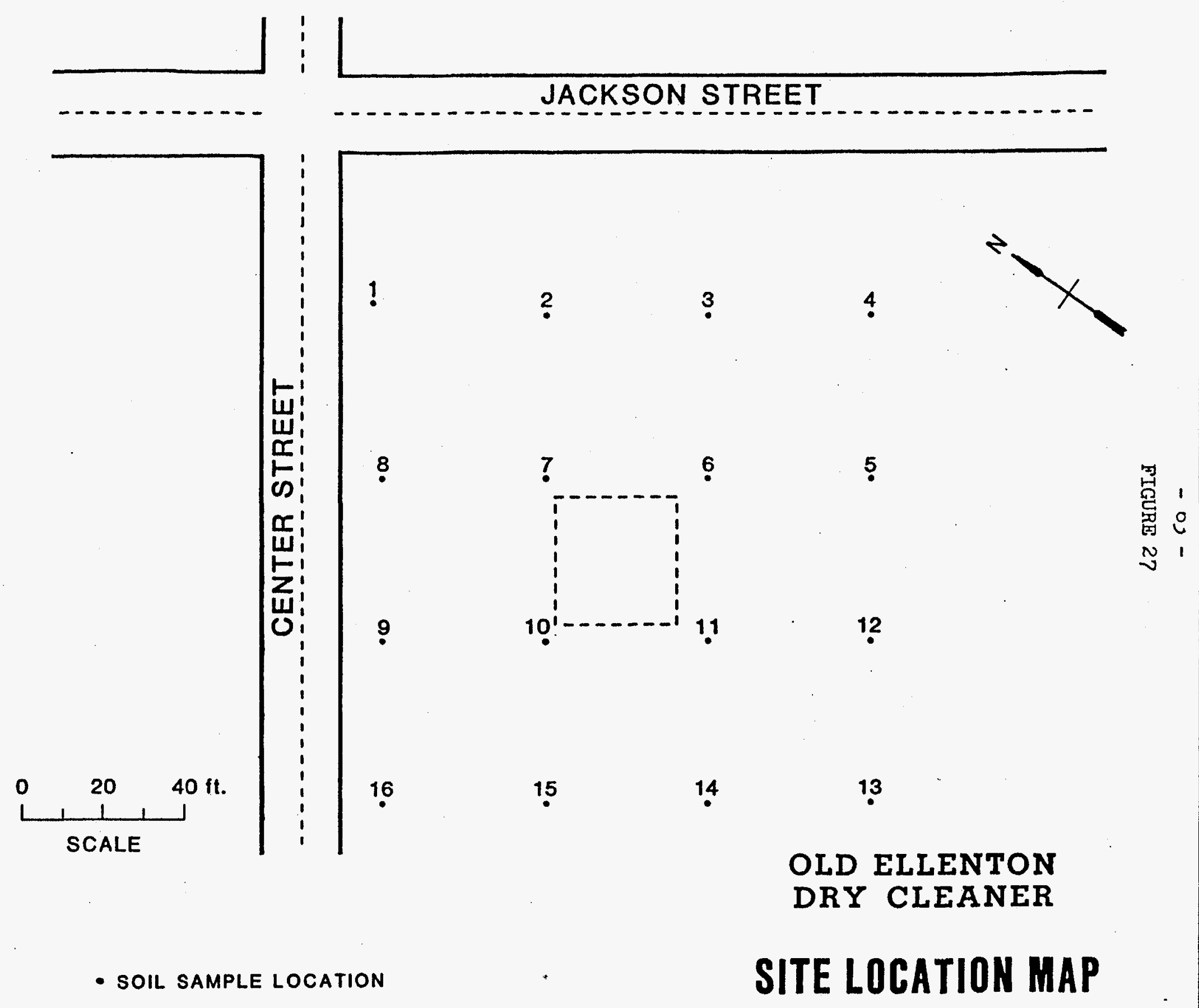

S.R.P. NOVEMBER, 1986 
--- E. I. DUPONT DE MEMOURS CD. INC., S.R.P., AIKEN, SC. ---

OLD ELLENTON - DRY CLEANERS -- 2 FT. SOIL SAMPLES, OCTOBER 1986 ---

--- SOIL CONCENTRATION BY NEIGHT IN PPB ----

\begin{tabular}{|c|c|c|c|c|c|c|c|c|c|c|c|c|}
\hline \multicolumn{2}{|c|}{$\begin{array}{l}\text { SAMFLE } \\
\text { NUKBERR }\end{array}$} & $\begin{array}{l}1,1-D i C l \\
\text { ETHYLEME }\end{array}$ & $\begin{array}{r}\text { DiCl } \\
\text { METHANE }\end{array}$ & $\begin{array}{r}\text { T1,2-DiCl } \\
\text { ETHYLEHE }\end{array}$ & $\begin{array}{r}1,1-D i C l \\
\text { ETHAHE }\end{array}$ & $\begin{array}{r}C 1,2-D i C l \\
\text { ETHYLENE }\end{array}$ & $\begin{array}{r}\text { TriCl } \\
\text { METHANE }\end{array}$ & $\begin{array}{r}\text { 1,2-DiCl } \\
\text { ETHANE }\end{array}$ & $\begin{array}{r}\text { TriCl } \\
\text { ETHYLEME }\end{array}$ & $\begin{array}{r}\text { JetraCl } \\
\text { ETHYLENE }\end{array}$ & & $\begin{array}{l}\text { YPLE } \\
\text { MBER }\end{array}$ \\
\hline$D C$ & 1 & - & - & - & - & - & - & - & - & - & DC & 1 \\
\hline DC & 2 & - & - & - & - & - & - & - & - & - & $D C$ & 2 \\
\hline$D C$ & 3 & - & - & - & - & - & - & - & - & - & DC & 3 \\
\hline$D C$ & 4 & - & - & - & - & - & - & - & - & - & OC & 4 \\
\hline$D C$ & 5 & - & - & - & - & - & - & - & - & - & $D C$ & 5 \\
\hline DC & 6 & - & - & - & - & - & - & - & - & - & DC & $b$ \\
\hline$D C$ & 7 & - & - & - & - & - & - & - & - & - & DC & 7 \\
\hline$D C$ & 8 & - & - & - & - & - & - & - & - & - & $D C$ & 8 \\
\hline$D C$ & 9 & - & - & - & - & - & - & - & - & - & $D C$ & 9 \\
\hline$D C$ & 10 & - & - & - & - & - & - & - & - & - & $D C$ & 10 \\
\hline$D C$ & 11 & - & -. & - & - & - & - & - & - & - & $D C$ & 11 \\
\hline$D C$ & 12 & - & - & - & - & - & - & - & - & - & DC & 12 \\
\hline$D C$ & 13 & - & - & - & - & - & - & - & - & - & $D C$ & 13 \\
\hline$D C$ & 14 & - & - & - & - & - & - & - & - & - & $D C$ & 14 \\
\hline$D C$ & 15 & - & - & - & - & - & - & - & - & - & $D C$ & 15 \\
\hline$D C$ & 16 & - & - & - & - & - & - & - & - & - & $D C$ & 16 \\
\hline
\end{tabular}

]

1

1

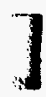

1

1

1 


\section{B. The Oil Test Site}

At the 0il Test Site, a total of sixty-five sites were

sampled for soil gas $C_{1}-C_{4}$ hydrocarbons and twelve soil samples were taken for gasoline range hydrocarbon analysis. Locations of these samples are shown on the Site Location Map in Figure 28.

We were informed that previous tests in this area had revealed that the "oil saturated" zone was generally confined to the upper foot of soil. We confirmed this observation in the hydraulic fluid plot and the oil test plots by digging several shallow trenches. With few exceptions, the upper 6-8" of soil was a dark brown color and contrasted sharply with the lower, apparently unaffected soils.

We conducted: soil gas depth tests to establish an optimum sampling depth. Soil gas samples were taken at 1, 2 and 3 foot depths at two oil test plots (sample locations 14 and 25 in Figure 28), and two control plots (sample locations 17 and 22 in Figure 28). Because the surface soils of both the oil test plots and the control plots appeared to have been roto-tilled, we collected both a $1 \mathrm{ft}$ and $3 \mathrm{ft}$ soil gas sample in an undisturbed soil zone (sample location $\|_{1}$ in Figure 28).

The results of the soil gas depth tests showed appreciably higher soil gas magnitudes at the $1 \mathrm{ft}$ level. Therefore, all other soil gas and soil samples in this area were taken from the $1 \mathrm{ft}$ level. 
Representative soil gas data is mapped in Figures 29-31 and all soil gas data is tabulated in Tables 16 and 17 . Both methane and the unsaturated compounds, ethylene and propylene, are products of biological activity. It is unlikely that either of these compounds was a component of the refined products placed in the soils in this area since all are gases at ambient temperatures. It is obvious, however, from the data that all three of these compounds have greater magnitudes in the soils of the contaminated plots than the soils of the control plots. This would appear to suggest a higher level of biological activity in the contaminated soils. It is noted that other hydrocarbons, such as butane (see Figure 31), which may not be products of biological activity, are also higher in the contaminated soils.

The distribution of ethylene and propylene in the soils between the test and control plots is further illustrated as frequency histograms in Figures 32,33 and 34 . The overall histogram in Figure 32 is clearly bimodal). This bimodality may be understood to be composed of the sum of a background population in the control plots, as shown in Figure 33, and an anomalous population in the test plots, as shown in Figure 34.

At twelve sites shown in Figure 28, soil samples were taken for gasoline range analysis. Although as shown on the chromatograms for Sites 25, 29 and 58 (see Figures 35, 36 and 37) there are numerous peaks, presumably corresponding to oil contamination, none of the peaks (retention times) correspond to the six 
gasoline range hydrocarbons monitored in this survey.

Identities of compounds could be ascertained using standard gas chromatograph-mass spectral techniques. 


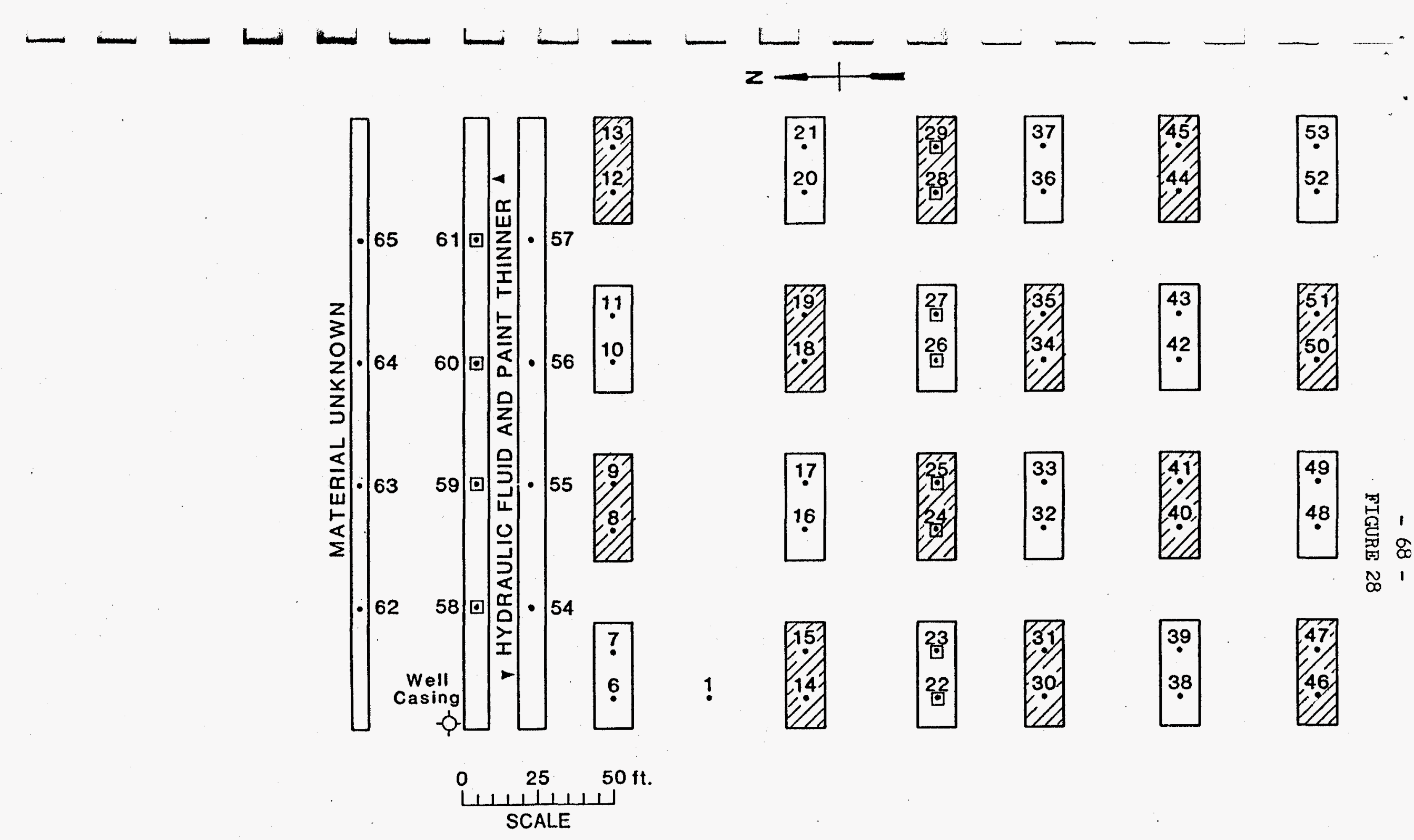

- SOIL gas SAMPLES

๑ SOIL GAS \& SOIL SAMPLES

$\square$ OIL TEST PLOT

$\square$ CONTROL TEST PLOT

S.R.P. OIL TEST SITE SITE LOCATION MAP

S.R.P. NOVEMBER, 1986 


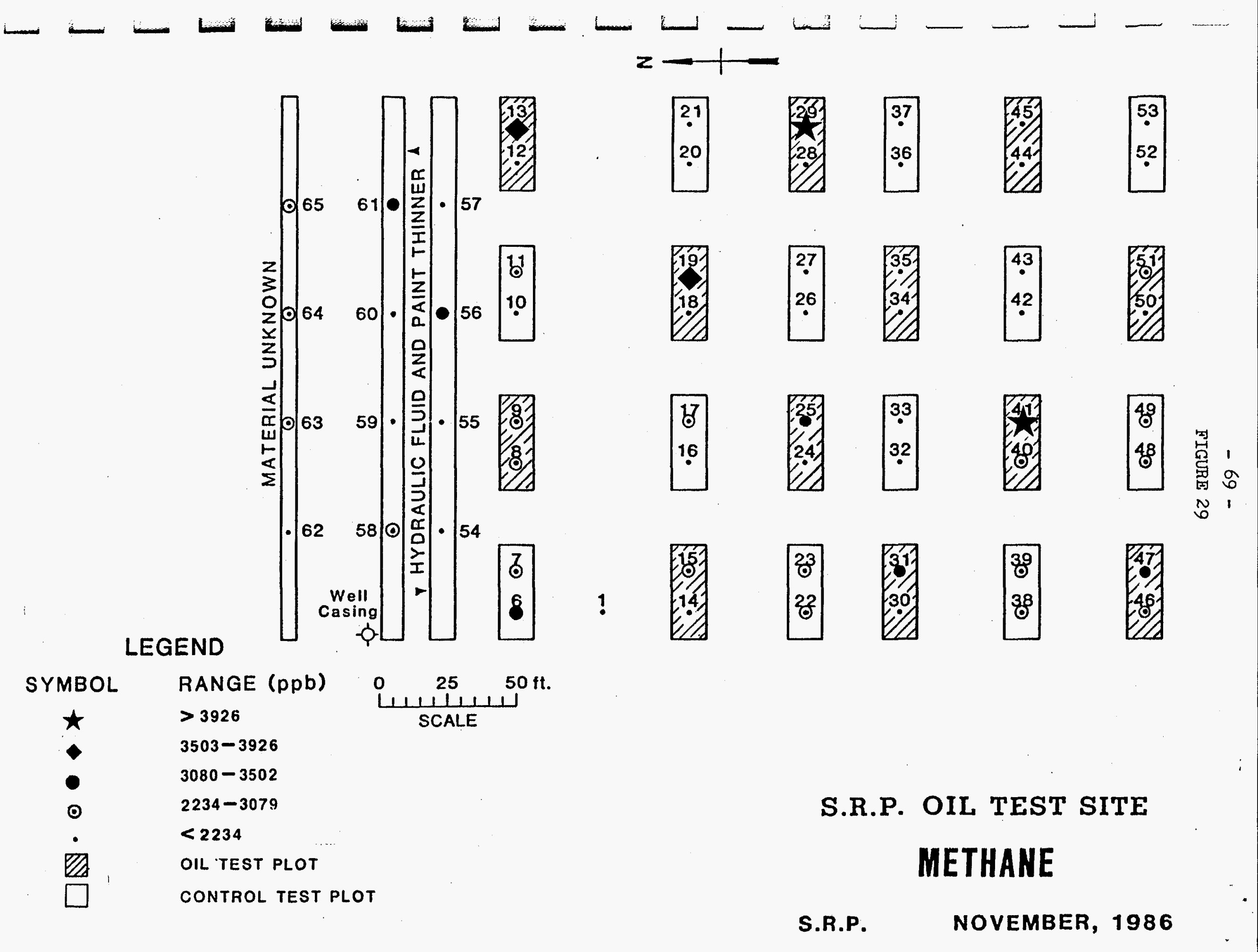




m. $n^{n}$. fis.

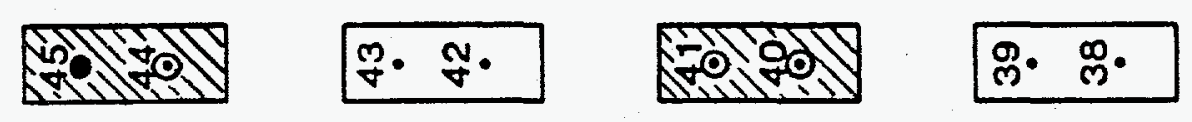

n.

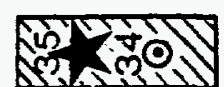

M. N.

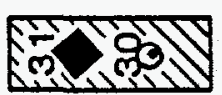

1

1
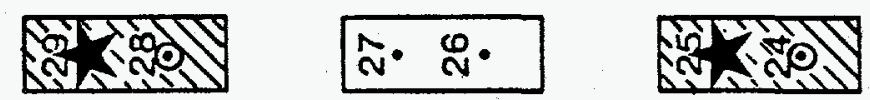

M. N.

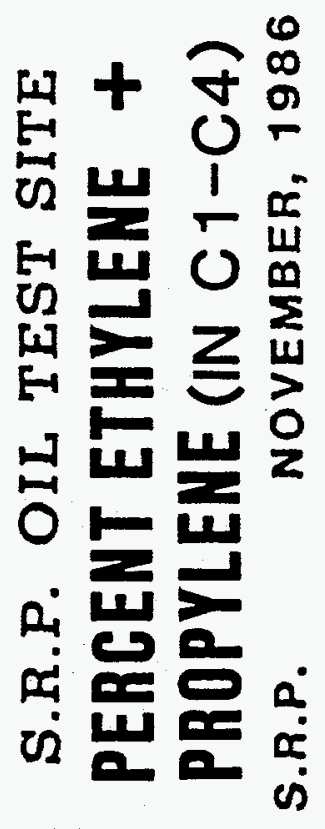

$1+$

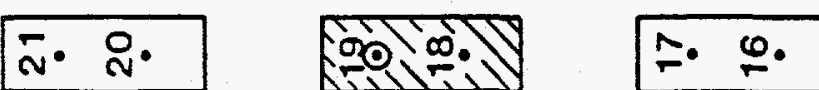

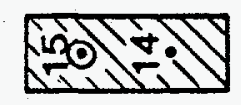

1

1
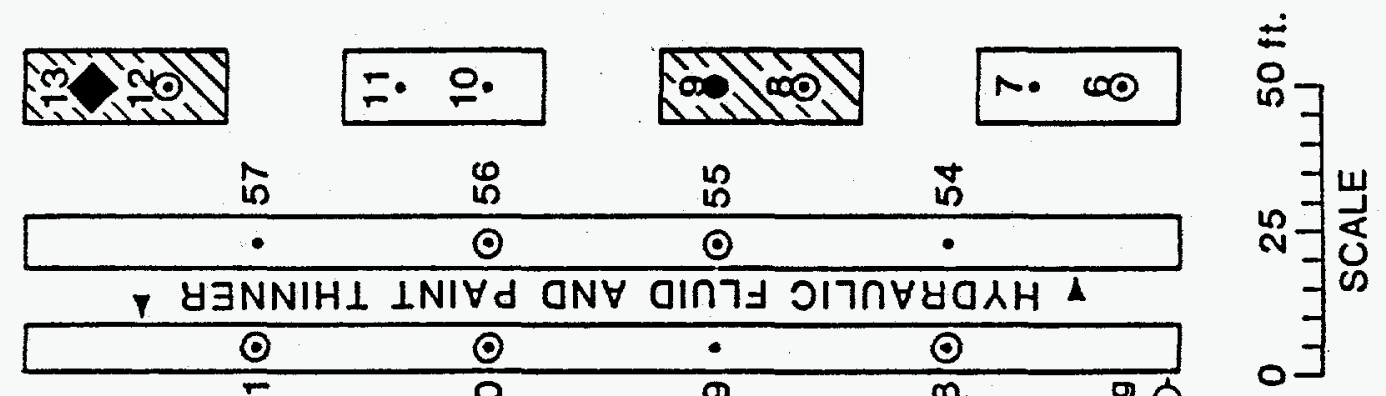

1

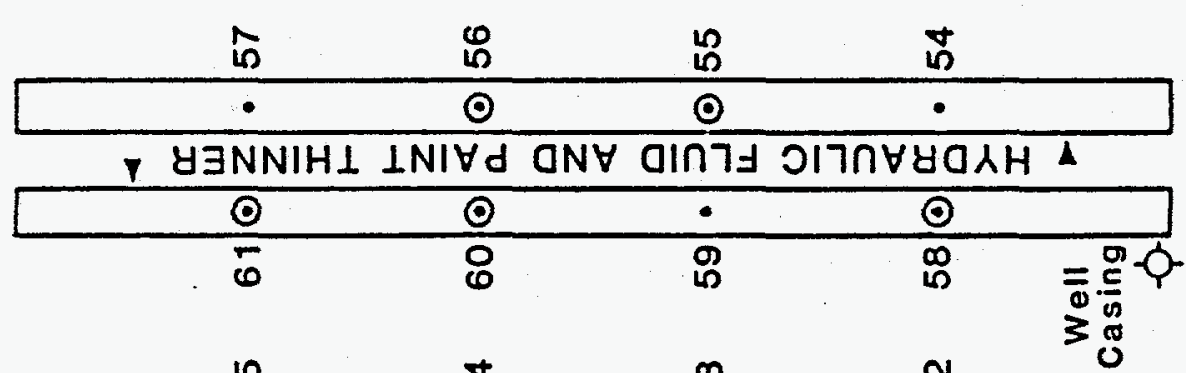

1

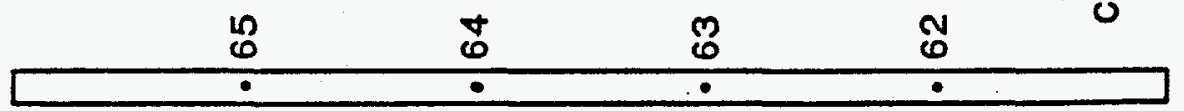

NMONXNก $7 \forall 18 \exists \perp \forall W$

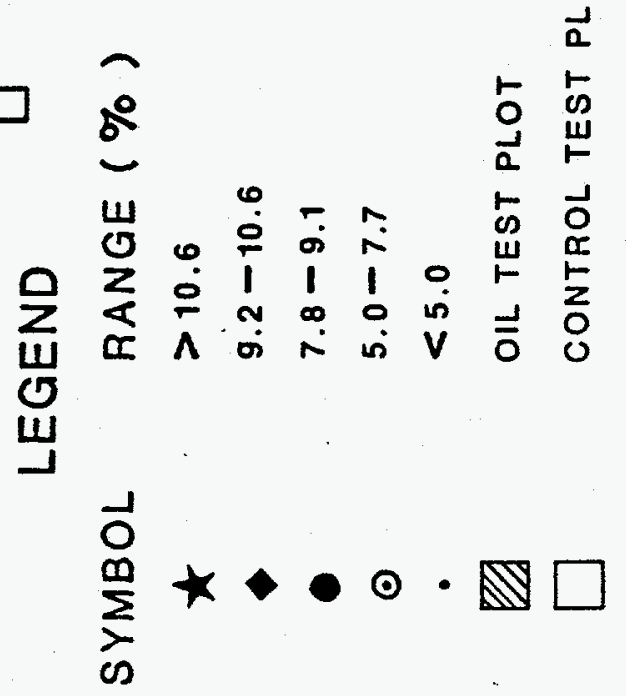



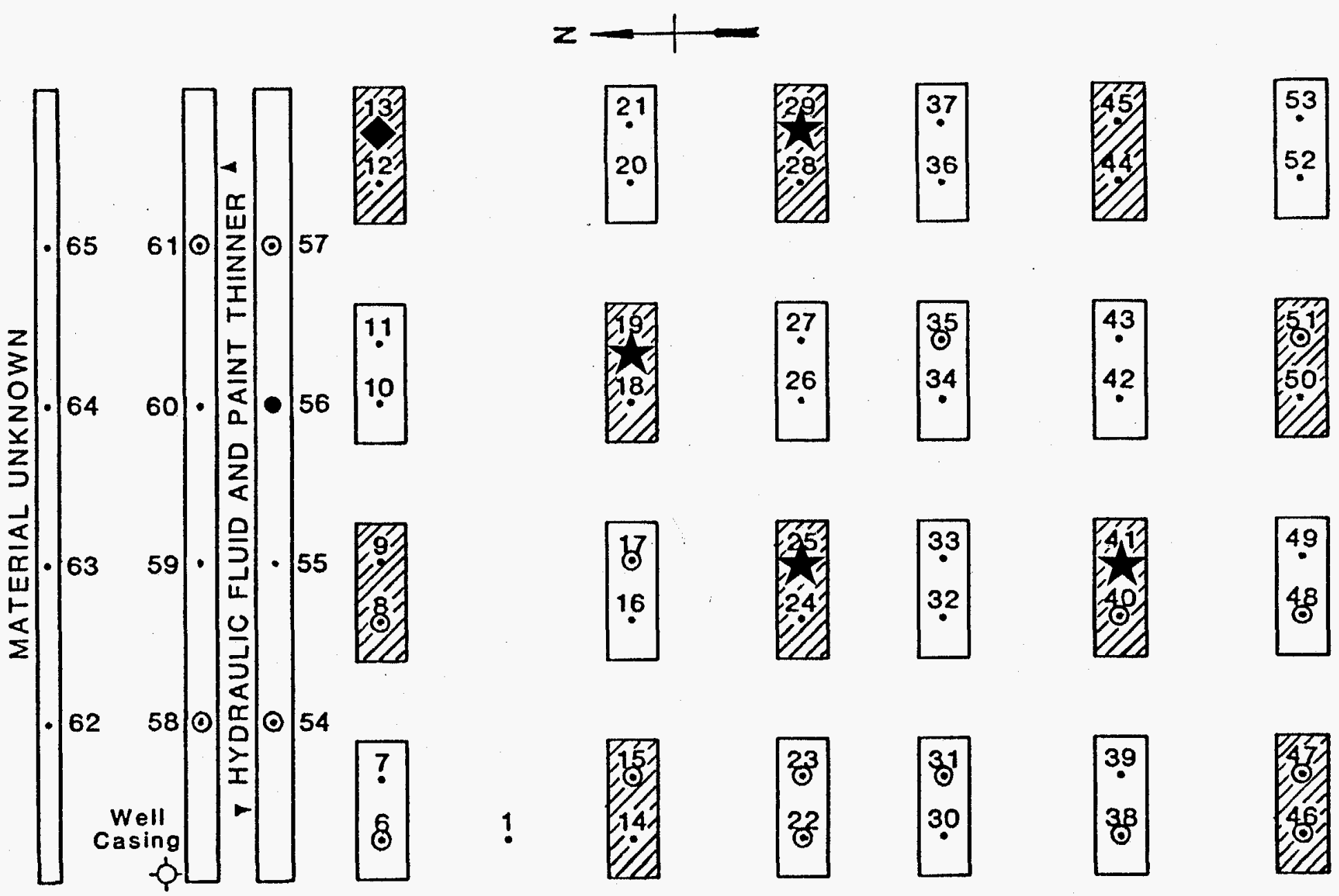

LEGEND
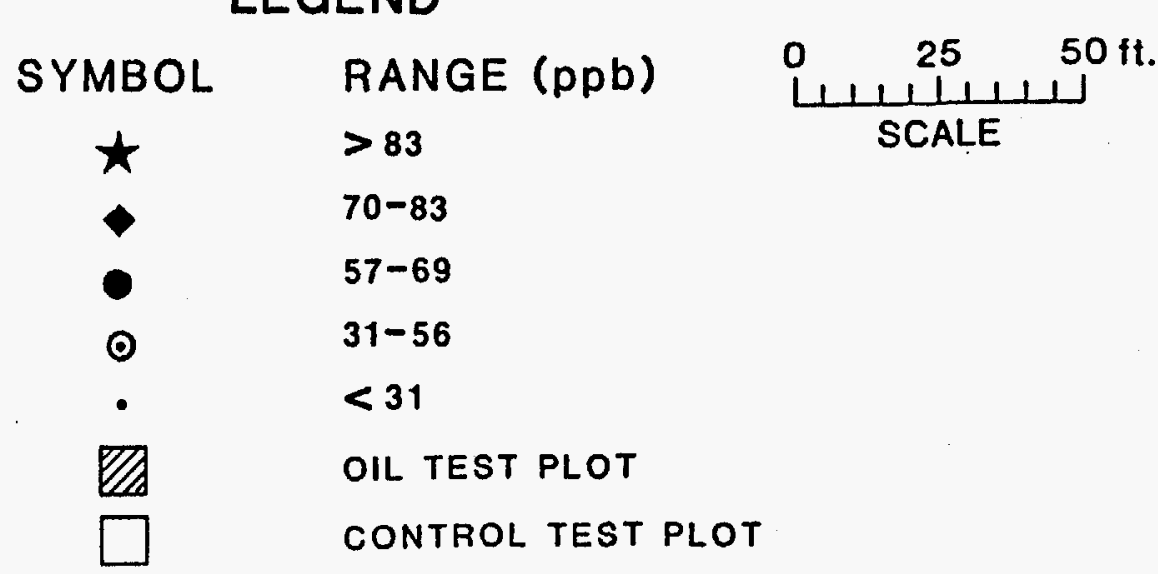

S.R.P. OIL TEST SITE

\section{BUTANE}

S.R.P.

NOVEMBER, 1986 


\section{OIL TEST SITE - HISTOGRAM}

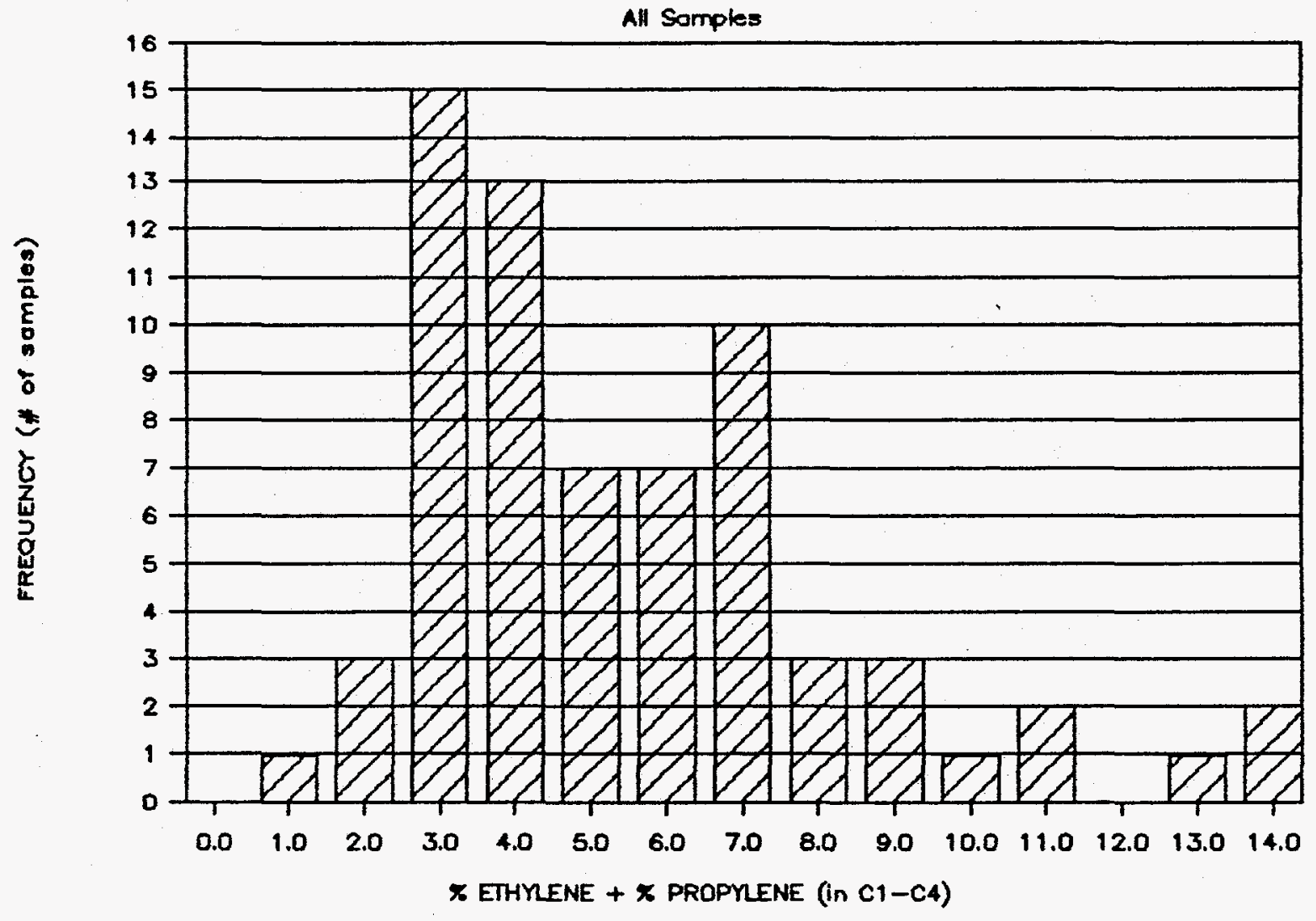


FIGURE $33 \& 34$

OIL TEST SITE - HISTOGRAM

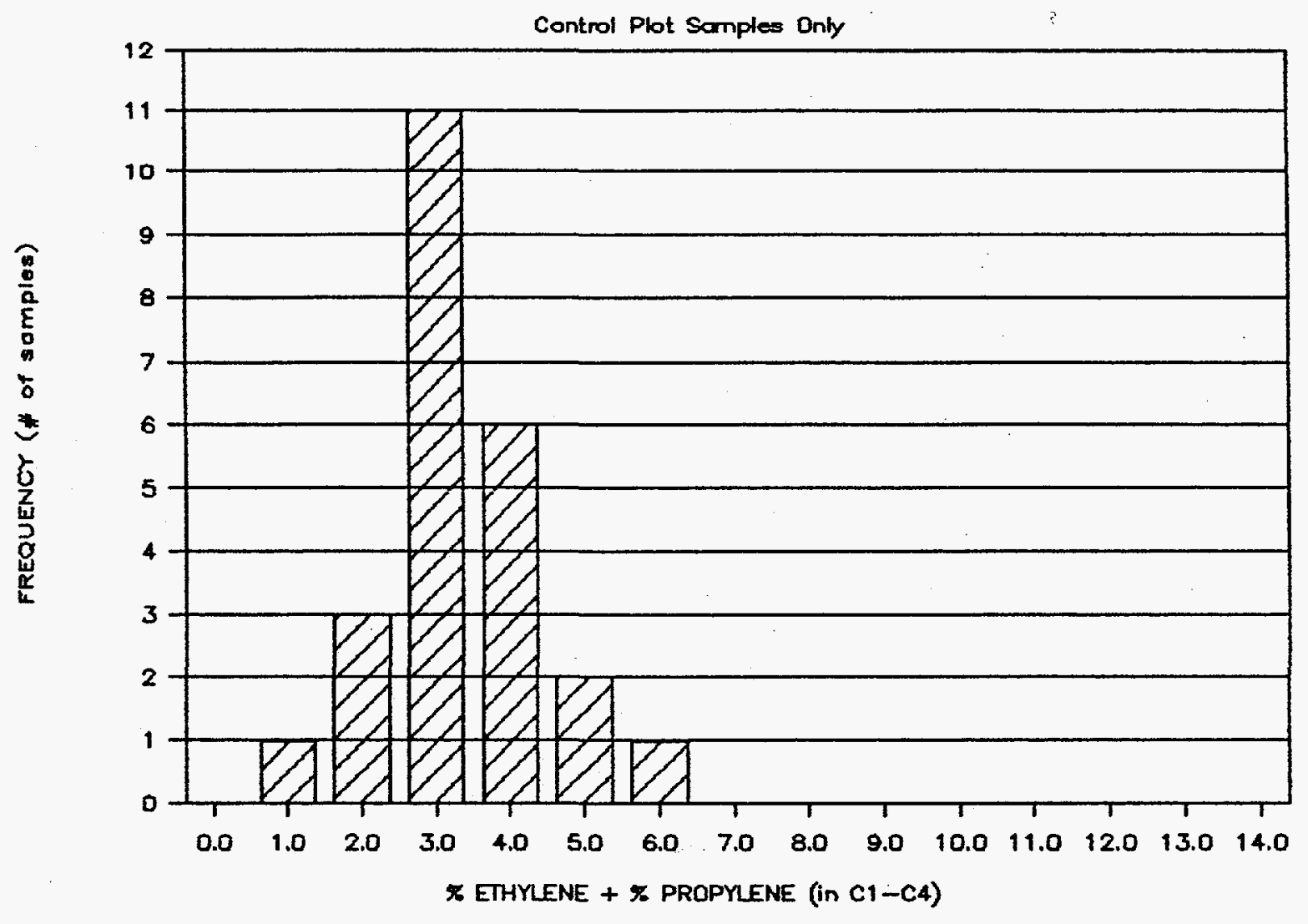

OIL TEST SITE - HISTOGRAM

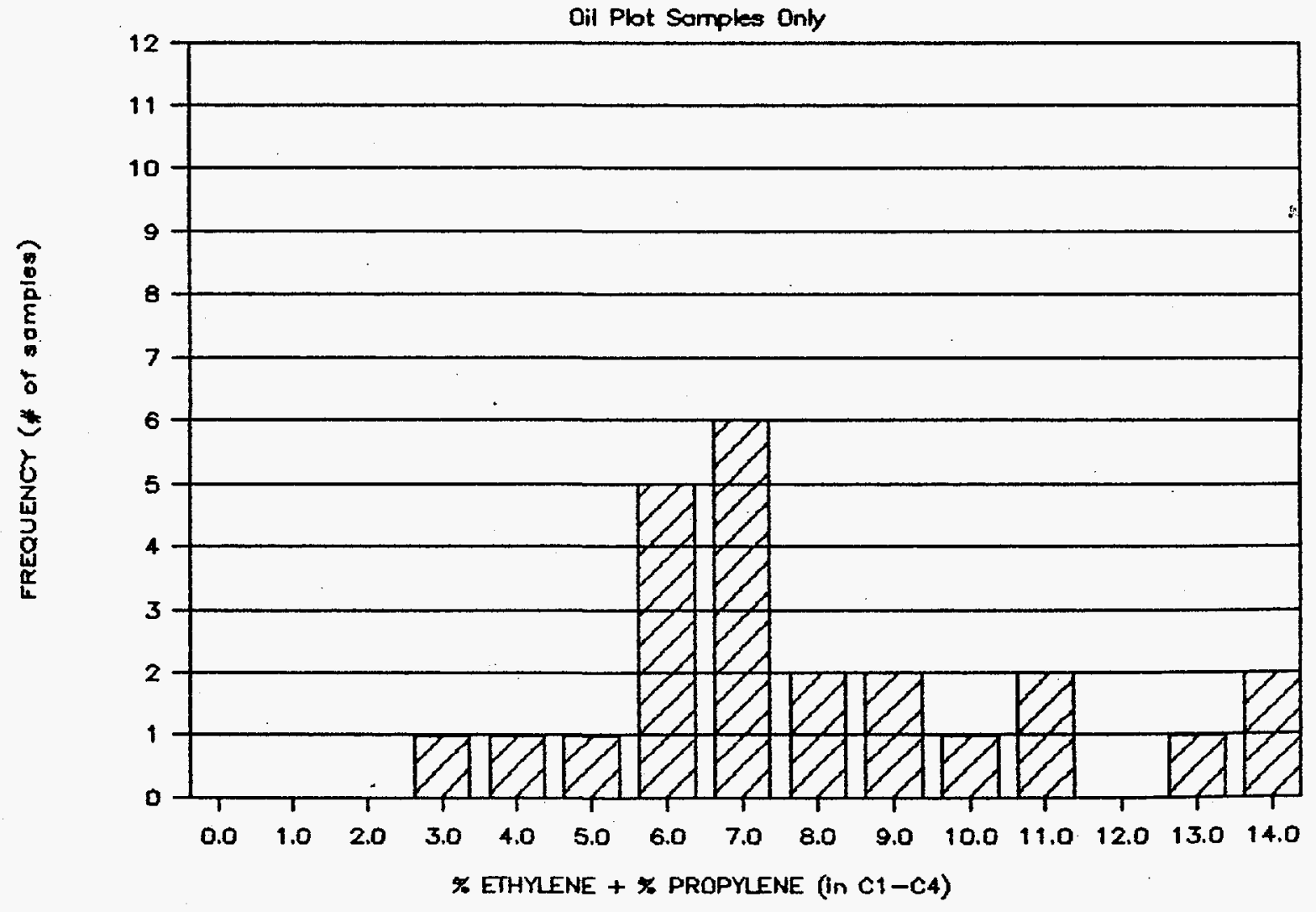


-.- E. I. DUPONT DE MENOURS CO. INC., S.R.P., AIKEN, S.C. -..-

--- OIL TEST SITE -- PROBE SURVEY, DCT. 19B6 --.-

-..- SOIL GAS CONCENTRAIIOKS -...

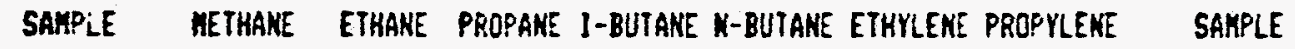

- PPB PPE PPB PPE PPB PPE PFE

\begin{tabular}{|c|c|c|c|c|c|c|c|c|c|c|}
\hline OT & $1-3$ & 893 & 23 & 9 & - & - & $1 t$ & 17 & OT & $1-3$ \\
\hline 01 & 6 & 3187 & 100 & 36 & 55 & - & 105 & 97 & DT & 6 \\
\hline or & 7 & 2422 & 57 & 20 & 28 & - & 37 & 37 & $0 T$ & 7 \\
\hline 07 & 8 & 2982 & 107 & 17 & 38 & - & 125 & 100 & OT & 8 \\
\hline OT & 9 & 2275 & 79 & 36 & 27 & - & 147 & 92 & OT & 9 \\
\hline OT & 10 & 2120 & 55 & 24 & 18 & - & 44 & 39 & $0 T$ & 10 \\
\hline OT & 11 & 2286 & 62 & 24 & 17 & - & 35 & 36 & OT & 11 \\
\hline $0 T$ & 12 & 2089 & 67 & 37 & 17 & - & 91 & 49 & $0 T$ & 12 \\
\hline 01 & 13 & 3537 & 176 & 68 & 47 & 26 & 273 & 158 & DT & 13 \\
\hline OT & 14 & 1688 & 26 & 12 & - & - & 22 & 18 & OT & 14 \\
\hline OT & $14-2$ & 1807 & 55 & 21 & 21 & - & 49 & 51 & OT & $14-2$ \\
\hline OT & $14-3$ & 1584 & 30 & 9 & - & - & 21 & 23 & $0 T$ & $14-3$ \\
\hline OI & 15 & 2489 & 100 & 38 & 40 & - & 99 & 87 & OT & 15 \\
\hline ot & 16 & 1730 & 53 & 17 & 17 & - & 44 & 37 & OT & 16 \\
\hline 01 & 17 & 2439 & 84 & 28 & 32 & - & 63 & $5 ?$ & OT . & 17 \\
\hline or & $17-2$ & 1066 & 45 & 15 & 20 & - & 28 & 31 & 07 & $17-2$ \\
\hline OT & $17-3$ & 713 & 30 & 11 & 15 & - & 21 & 22 & OT & $17-3$ \\
\hline OT & 18 & 2108 & 62 & 25 & 25 & - & 56 & 51 & OT & 18 \\
\hline 07 & 19 & 3754 & 174 & 63 & 60 & 25 & 164 & 142 & 0T & 19 \\
\hline OT & 20 & 1628 & 21 & 9 & 16 & - & 15 & 22 & DT & 20 \\
\hline 01 & 21 & 1857 & 35 & 13 & 24 & - & 25 & 24 & OT & 21 \\
\hline DT & 22 & $22 \mathrm{sg}$ & 63 & 21 & 31 & - & 35 & 54 & OT & 22 \\
\hline OT & $22-2$ & 781 & 21 & 7 & - & - & 14 & 13 & OT & $22-2$ \\
\hline or & 23 & 2551 & 81 & 24 & 36 & - & 42 & 45 & or & 23 \\
\hline 01 & 24 & 1804 & 50 & 22 & 18 & - & 78 & 58 & OT & 24 \\
\hline oT & 25 & 3342 & 127 & 56 & 53 & 23 & $19 t$ & 153 & OT & 25 \\
\hline $0 !$ & $25-k$ & 3207 & 175 & 76 & 90 & 32 & $32 i$ & $24 i$ & OT & $25-R$ \\
\hline OT & $25-2$ & 1243 & 20 & 7 & 11 & - & 25 & 20 & OT & $25-2$ \\
\hline OT & $25-3$ & 1152 & 25 & 10 & 15 & - & 21 & 22 & OT & $25-3$ \\
\hline OT & 26 & $140 ?$ & 17 & - & - & - & 9 & - & 0! & 26 \\
\hline OT & 27 & 2067 & 39 & 13 & 17 & - & 21 & 23 & DT & 27 \\
\hline OT & 28 & 2051 & 53 & 26 & 24 & - & 98 & 67 & OI & 28 \\
\hline $0 T$ & 29 & 4382 & 237 & 96 & 81 & 31 & $45 t$ & 285 & DT & 29 \\
\hline 01 & 30 & 2184 & 77 & 33 & 27 & - & 67 & 70 & OI & 30 \\
\hline 07 & 31 & 3147 & 186 & 70 & 50 & 1 & 217 & 181 & OT & 31 \\
\hline $0 T$ & 32 & 1682 & 22 & 8 & 12 & - & 15 & 18 & OT & 32 \\
\hline OT & 33 & 1467 & 11 & - & - & - & 10 & 12 & OT & 33 \\
\hline OT & 34 & 2139 & 70 & 27 & 25 & - & 68 & 55 & OT & 34 \\
\hline DT & 35 & 1680 & 83 & 35 & 36 & - & 162 & 110 & oT & 35 \\
\hline OT & 36 & 1652 & 19 & 17 & 23 & - & 30 & 31 & ot & 36 \\
\hline OT & 37 & 1356 & 30 & 10 & 15 & - & 19 & 21 & OT & 37 \\
\hline OT & 38 & 2592 & 70 & 22 & 34 & - & 36 & 37 & or & 38 \\
\hline OT & 39 & 2439 & 50 & 16 & 21 & - & 25 & 26 & of & 39 \\
\hline DT & 40 & 2905 & 126 & 47 & 49 & - & 124 & 106 & OT & 40 \\
\hline DT & 41 & 5729 & 274 & 97 & 87 & 34 & 287 & 222 & oT & 41 \\
\hline OT & 42 & 1314 & 31 & 11 & 14 & - & 18 & 29 & 01 & 42 \\
\hline $0 \mathrm{~T}$ & 43 & 1555 & 34 & 12 & 15 & - & 23 & 28 & OT & 43 \\
\hline DT & 44 & 1562 & 54 & 22 & 25 & - & 47 & 44 & OT & 44 \\
\hline OT & 45 & 1926 & 70 & 34 & 23 & - & 112 & 74 & DT & 45 \\
\hline
\end{tabular}




$$
\text { TABLE } 16 \text { (cont) }
$$

--- E. I. DUPONT DE MENOURS CO. INC., S.R.P., AIKEX, S.C. -..-

-..- OIL TEST SITE -- PROBE SURVEY, OCT. 1986 -..-

-.-- SOIL GAS COKCEXTRATIONS -...-

\begin{tabular}{|c|c|c|c|c|c|c|c|c|c|c|}
\hline \multicolumn{2}{|c|}{ SAMPLE } & $\begin{array}{r}\text { METHANE } \\
\text { PFE }\end{array}$ & $\begin{array}{r}\text { ETHANE } \\
\text { PPB }\end{array}$ & $\begin{array}{r}\text { PROPANE } \\
\text { PPE }\end{array}$ & $\begin{array}{c}\text { I-BUTANE } \\
\text { PPB }\end{array}$ & $\begin{array}{r}\text { N-BUTANE } \\
\text { PPB }\end{array}$ & $\begin{array}{r}\text { ETHYLEME } \\
\text { PPB }\end{array}$ & $\begin{array}{c}\text { PROPYLENE } \\
\text { PPB }\end{array}$ & \multicolumn{2}{|c|}{$\begin{array}{c}\text { SAMPLE } \\
1\end{array}$} \\
\hline סT & 48 & 2751 & 93 & $3 t$ & 51 & - & 103 & 98 & OT & 46 \\
\hline $0 T$ & 47 & 3147 & 124 & 15 & 55 & - & 100 & 99 & DT & 47 \\
\hline OT & 48 & 2979 & 76 & 25 & 36 & - & 39 & 42 & OT & 48 \\
\hline OI & 49 & 2455 & 50 & 16 & 23 & - & 25 & 30 & OT & 49 \\
\hline OT & 50 & 2061 & 50 & 20 & 21 & - & 39 & 34 & OT & 50 \\
\hline OT & 51 & 2252 & 86 & 38 & 37 & - & 145 & 104 & סT & 51 \\
\hline OT & 52 & 1666 & 43 & 15 & 17 & - & 22 & 25 & OT & 52 \\
\hline 01 & 53 & 1547 & 37 & 12 & 15 & - & 19 & 23 & $D T$ & 53 \\
\hline 07 & 54 & 2151 & 78 & 27 & 39 & - & 58 & 52 & $O T$ & 54 \\
\hline DT & 55 & 1401 & 38 & 18 & 16 & - & 64 & 44 & OT & 55 \\
\hline OT & 56 & 3279 & 129 & 46 & 63 & - & 135 & 97 & $D T$ & 56 \\
\hline OT & 57 & 2181 & 64 & 22 & 31 & - & 34 & 31 & $0 T$ & 57 \\
\hline OT & 58 & 3059 & 134 & 60 & 16 & - & 141 & 89 & OT & 58 \\
\hline OT & 59 & 2142 & 56 & 22 & 27 & - & 44 & 39 & DT & 59 \\
\hline OT & 60 & 1844 & 78 & 29 & 22 & - & 79 & 62 & DT & 60 \\
\hline OT & 61 & 3244 & 146 & 51 & 44 & - & 172 & 116 & OT & 61 \\
\hline OT & 62 & 2129 & 54 & 18 & 28 & - & 32 & 30 & DT & 62 \\
\hline OT & 63 & 2338 & 66 & 29 & 19 & - & 61 & 45 & OT & 63 \\
\hline OT & 64 & 2927 & 99 & 37 & 26 & - & 63 & 62 & OT & 64 \\
\hline OT & 65 & 2337 & 62 & 30 & 19 & - & 44 & 36 & OT & 65 \\
\hline
\end{tabular}

NOTE:

$$
\begin{aligned}
& \text { of } X X-1 F i \text {. SAMPLE } \\
& \text { OT } x(x-2-2 F: \text {. SAFFLE } \\
& \text { OT } X)-3 \text { - } 3 \text { F.. SAKFLE } \\
& \text { oi } X X-R \text { - REPEAT SAMFLE }
\end{aligned}
$$


-..- E. I. DUPONT DE MEMOURS CO. INC., S.R.P., AIKEN, S.C. - -

--. OIL TEST SITE - PROBE SURVEY, OCT. 1986 - -

-..- SOIL GAS PERCENTAGES

\begin{tabular}{|c|c|c|c|c|c|c|c|c|c|c|c|}
\hline $\begin{array}{c}\text { SAMPLE } \\
1\end{array}$ & $\begin{array}{l}\text { TOTAL } \\
\text { CI-C4 }\end{array}$ & $\begin{array}{r}\text { METHANE } \\
\text { ZCI }\end{array}$ & $\begin{array}{r}\text { ETHANE } \\
\text { IC2 }\end{array}$ & $\begin{array}{r}\text { PFOPANE } \\
\text { ICJ }\end{array}$ & $\begin{array}{l}\text { BUTANE } \\
\text { ICA }\end{array}$ & $\begin{array}{l}\text { TOTAL } \\
\text { HYDCARB }\end{array}$ & $\begin{array}{c}\text { ETHYLENE } P \\
z\end{array}$ & $\begin{array}{c}\text { PROPYLEKE } \\
\text { I }\end{array}$ & $\begin{array}{r}\text { SAMPL } \\
\quad\end{array}$ & & $\begin{array}{l}\text { SITE } \\
\text { TYPE }\end{array}$ \\
\hline OT $\quad 1-3$ & 925 & 96.5 & 2.5 & 1.0 & 0.0 & 958 & 1.7 & 1.8 & OT & $1-3$ & BK.6 \\
\hline OT 6 & 3378 & 94.3 & 3.0 & 1.1 & 1.6 & 3580 & 2.9 & 2.7 & OT & 6 & CTR \\
\hline от 7 & 2527 & 95.8 & 2.3 & 0.8 & 1.1 & 2601 & 1.4 & 1.4 & OT & 7 & CTK \\
\hline or 8 & 3174 & 94.0 & 3.4 & 1.5 & 1.2 & 3399 & 3.7 & 2.9 & OT & 8 & OIL \\
\hline or 9 & 2417 & 94.1 & 3.3 & 1.5 & 1.1 & 2656 & 5.5 & 3.5 & OT & 9 & OIL \\
\hline or 10 & 2217 & 95.6 & 2.5 & 1.1 & 0.8 & 2300 & 1.9 & 1.7 & OT & 10 & ICTR \\
\hline OT 11 & 2389 & 95.7 & 2.6 & 1.0 & 0.7 & 2460 & 1.4 & 1.5 & $0 T$ & 11 & CTR \\
\hline OT 12 & 2210 & 94.5 & 3.0 & 1.7 & 0.8 & 2350 & 3.9 & 2.1 & $0 T$ & 12 & OIL \\
\hline or 13 & 3854 & 91.8 & 4.6 & 1.8 & 1.9 & 4285 & 6.4 & 3.7 & $O T$ & 13 & Oll \\
\hline OT 14 & 1726 & 97.8 & 1.5 & 0.7 & 0.0 & 1766 & 1.2 & 1.0 & OT & 14 & OIL \\
\hline or $14-2$ & 1904 & 94.9 & 2.9 & 1.1 & 1.1 & 2004 & 2.4 & 2.5 & OT & $14-2$ & OIL \\
\hline of $14-3$ & 1623 & 97.6 & 1.8 & 0.6 & 0.0 & 1667 & 1.3 & 1.4 & $0 T$ & $14-3$ & OIL \\
\hline OT 15 & 2667 & 93.3 & 3.7 & 1.4 & 1.5 & 2853 & 3.5 & 3.0 & OT & 15 & OIL \\
\hline DT 16 & 1817 & 95.2 & 2.9 & 0.9 & 0.9 & 1898 & 2.3 & 1.9 & OI & 16 & ICTR \\
\hline oT 17 & 2583 & 94.4 & 3.3 & 1.1 & 1.2 & 2699 & 2.3 & 2.0 & $0 T$ & 17 & ICTf \\
\hline of $17-2$ & 1146 & 93.0 & 3.9 & 1.3 & 1.7 & 1205 & 2.3 & 2.6 & OT & $17-2$ & CTR \\
\hline or $17-3$ & 769 & 92.7 & 3.9 & 1.4 & 2.0 & 812 & 2.6 & 2.7 & OT & $17-3$ & CTR \\
\hline OT 18 & 2220 & 95.0 & 2.8 & 1.1 & 1.1 & 2327 & 2.4 & 2.2 & $0 T$ & 18 & OIL \\
\hline OT 19 & 4076 & 92.1 & 4.3 & 1.5 & 2.1 & 1382 & 3.7 & 3.2 & OT & 19 & OIL \\
\hline oT 20 & 1674 & 97.3 & 1.3 & 0.5 & 1.0 & 1711 & 0.9 & 1.3 & OT & 20 & CTK \\
\hline OT 21 & 1929 & 96.3 & 1.8 & 0.7 & 1.2 & 1978 & 1.3 & 1.2 & OT & 21 & $*$ CTR \\
\hline OT 22 & 2374 & 95.2 & 2.7 & 0.9 & 1.3 & 2463 & 1.4 & 2.2 & OT & 22 & CTR \\
\hline oT $22-2$ & 809 & 96.5 & 2.6 & 0.9 & 0.0 & 836 & 1.7 & 1.6 & OT & $22-2$ & CTR \\
\hline oT 23 & 2692 & 94.8 & 3.0 & 0.9 & 1.3 & 2779 & 1.5 & 1.6 & OT & 23 & CTR \\
\hline OT 24 & 1894 & 95.2 & 2.6 & 1.2 & 1.0 & 2030 & 3.8 & 2.9 & OT & 24 & OIL \\
\hline OT 25 & 3601 & 92.8 & 3.5 & 1.6 & 2.1 & 3950 & 5.0 & 3.9 & OT & 25 & DIL \\
\hline (i) $25-R$ & 3580 & 89.6 & 4.9 & 2.1 & 3.4 & 4142 & 7.7 & 5.8 & OT & $25-K$ & OIL \\
\hline oा $25-2$ & 1281 & 97.0 & 1.6 & 0.5 & 0.9 & 1326 & 1.9 & 1.5 & 07 & $25-2$ & OIL \\
\hline oT $25-3$ & 1202 & 95.8 & 2.1 & 0.8 & 1.2 & 1245 & 1.7 & 1.8 & $0 T$ & $25-3$ & OIL \\
\hline oT 26 & 1124 & 98.8 & 1.2 & 0.0 & 0.0 & 1433 & 0.6 & 0.0 & OT & 26 & CTR \\
\hline OT 27 & 2136 & 96.8 & 1.8 & 0.6 & 0.8 & 2180 & 1.0 & 1.1 & DT & 27 & CTR \\
\hline OT 28 & 2154 & 95.2 & 2.5 & 1.2 & 1.1 & 2319 & 4.2 & 2.9 & $0 T$ & 28 & OIL \\
\hline OT 29 & 1827 & 90.8 & 4.9 & 2.0 & 2.3 & 5568 & 8.2 & 5.1 & OT & 29 & OIL \\
\hline ot 30 & 2321 & 94.1 & 3.3 & 1.4 & 1.2 & 2458 & 2.7 & 2.8 & $B T$ & 30 & OIL \\
\hline OT 31 & 3457 & 91.0 & 5.4 & 2.0 & 1.6 & 3858 & 5.6 & 4.8 & $0 T$ & 31 & OIL \\
\hline oT 32 & 1724 & 97.6 & 1.3 & 0.5 & 0.7 & 1757 & 0.9 & 1.0 & OT & 32 & CTR \\
\hline ot 33 & 1476 & 99.3 & 0.7 & 0.0 & 0.0 & 1500 & 0.7 & 0.8 & OT & 33 & CTR \\
\hline OT 31 & 2261 & 94.6 & 3.1 & 1.2 & 1.1 & 2384 & 2.9 & 2.3 & OT & 34 & OIL \\
\hline ot 35 & 1834 & 91.6 & 4.5 & 1.9 & 2.0 & 2106 & 7.7 & 5.2 & OT & 35 & OIL \\
\hline OT 36 & 1741 & 94.9 & 2.8 & 1.0 & 1.3 & 1802 & 1.7 & 1.7 & OT & 36 & CTR \\
\hline of 37 & 1411 & 96.1 & 2.1 & 0.7 & 1.1 & 1451 & 1.3 & 1.4 & DT & 37 & CTR \\
\hline OT 38 & 2718 & 95.4 & 2.6 & 0.8 & 1.3 & 2791 & 1.3 & 1.3 & OF & 38 & CTR \\
\hline or 39 & 2526 & 96.6 & 2.0 & 0.6 & 0.8 & 2577 & 1.0 & 1.0 & DT & 39 & CTR \\
\hline DT 40 & 3127 & 92.9 & 4.0 & 1.5 & 1.6 & 3357 & 3.7 & 3.2 & OT & 40 & OIL \\
\hline OT 11 & 6221 & 92.1 & 4.4 & 1.6 & 1.9 & 6730 & 4.3 & 3.3 & OT & 41 & OIL \\
\hline oT 12 & 1370 & 95.9 & 2.3 & 0.8 & 1.0 & 1417 & 1.3 & 2.0 & OT & 12 & CTR \\
\hline or 43 & 1616 & 96.2 & 2.1 & 0.7 & 0.9 & 1667 & 1.4 & 1.7 & OT & 43 & CTR \\
\hline OT 41 & 1663 & 93.9 & 3.2 & 1.3 & 1.5 & 1754 & 2.7 & 2.5 & OT & 44 & OIL \\
\hline OT 15 & 2053 & 93.8 & 3.4 & 1.7 & 1.1 & 2239 & 5.0 & 3.3 & OT & 45 & OIL \\
\hline
\end{tabular}


TABLE 17 (cont)

-.- E. I. DUPONT DE MENOURS CO. INC., S.R.P., AIKEN, S.C. -

OIL IEST SITE - PROEE SURVEY, OCT. 1986 -

---- SOIL GAS PERCENTAGES

\begin{tabular}{|c|c|c|c|c|c|c|c|c|c|c|c|}
\hline $\begin{array}{c}\text { SAMPLE } \\
1\end{array}$ & $\begin{array}{l}\text { TOTAL } \\
\text { CI-CA }\end{array}$ & $\begin{array}{r}\text { METHANE } \\
\text { \%C! }\end{array}$ & $\begin{array}{r}\text { ETHANE } \\
\text { ICZ }\end{array}$ & $\begin{array}{r}\text { PROPANE } \\
\text { ICJ }\end{array}$ & $\begin{array}{l}\text { BUTANE } \\
\text { XC4 }\end{array}$ & $\begin{array}{c}\text { TOTAL } \\
\text { HYDCARB }\end{array}$ & $\begin{array}{r}\text { ETHYLENE } \\
q\end{array}$ & $\begin{array}{c}\text { YLENE } \\
?\end{array}$ & \multicolumn{2}{|c|}{$\begin{array}{c}\text { SAIRPLE } \\
1\end{array}$} & $\begin{array}{l}\text { SITE } \\
\text { TYPE }\end{array}$ \\
\hline ot 46 & 2931 & 93.9 & 3.2 & 1.2 & 1.7 & 3132 & 3.3 & 3.1 & $0 T$ & 46 & OIL \\
\hline OT 47 & $337 !$ & 93.4 & 3.7 & 1.3 & 1.6 & 3570 & 2.8 & 2.8 & OT & 47 & OIL \\
\hline OT 48 & 3116 & 95.6 & 2.4 & 0.8 & 1.2 & 3197 & 1.2 & 1.3 & DT & 48 & CTR \\
\hline OT 49 & 2544 & 96.5 & 2.0 & 0.6 & 0.9 & 2599 & 1.0 & 1.2 & OT & 49 & CTR \\
\hline or 50 & 2152 & 95.8 & 2.3 & 0.9 & 1.0 & 2225 & 1.8 & 1.5 & OT & 50 & OIL \\
\hline oT 51 & 2413 & 93.3 & 3.6 & 1.6 & 1.5 & 2662 & 5.4 & 3.9 & OT & 51 & OIL \\
\hline OT 52 & 1741 & 95.7 & 2.5 & 0.9 & 1.0 & 1788 & 1.2 & 1.4 & OT & 52 & CTR \\
\hline OT 53 & 1611 & 96.0 & 2.3 & 0.7 & 0.9 & 1653 & 1.1 & 1.4 & OT & 53 & CTR \\
\hline oT 54 & 2295 & 93.7 & 3.4 & 1.2 & 1.7 & 2405 & 2.4 & 2.2 & OT & 54 & $H \& P$ \\
\hline OT 55 & 1473 & 95.1 & 2.6 & 1.2 & 1.1 & 1581 & 4.0 & 2.8 & $0 T$ & 55 & H\&F \\
\hline OT 56 & 3517 & 93.2 & 3.7 & 1.3 & 1.8 & 3749 & 3.6 & 2.6 & OT & 56 & HEF \\
\hline OT 57 & 2298 & 94.9 & 2.8 & 1.0 & 1.3 & 2363 & 1.4 & 1.3 & OT & 57 & H\&P \\
\hline or 58 & 3299 & 92.7 & 4.1 & 1.8 & 1.4 & 3525 & 4.0 & 2.5 & or & 58 & HLP \\
\hline 0159 & 2247 & 95.3 & 2.5 & 1.0 & 1.2 & 2330 & 1.9 & 1.7 & OT & 59 & H\&P \\
\hline OT 60 & 1973 & 93.5 & 4.0 & 1.5 & 1.1 & 2114 & 3.7 & 2.9 & OT & 60 & $H \& P$ \\
\hline 0161 & 3485 & 93.1 & 4.2 & 1.5 & 1.3 & 3773 & 4.6 & 3.1 & DT & 61 & $H \neq P$ \\
\hline OT 62 & 2229 & 95.5 & 2.4 & 0.8 & 1.3 & 2291 & 1.4 & 1.3 & $O T$ & $62^{\circ}$ & UNK \\
\hline 0163 & 2452 & 95.1 & 2.7 & 1.2 & 0.8 & 2558 & 2.4 & 1.8 & 01 & 63 & UHF: \\
\hline OT 64 & 3099 & 94.8 & 3.2 & 1.2 & 0.8 & 3214 & 2.0 & 1.9 & OT & 64 & UNR: \\
\hline OT 65 & 2448 & 95.5 & 2.5 & 1.2 & 0.8 & 2528 & 1.7 & 1.4 & OT & 65 & UWK. \\
\hline
\end{tabular}

NOTE:

$$
\begin{aligned}
& \text { OT } X X \quad-1 \text { FT. SAMFE } \\
& \text { OT } x x-2-2 \text { FT. SAME E } \\
& \text { OT } X X-3 \text { - 3FT. SAEF LE } \\
& \text { OT } X X-R \text { - REPEAT SAKILE } \\
& \text { OIL - OIL SITE } \\
& \text { CTR - CONTROL SITE } \\
& \text { HLP - HYDRALLIC FLUIO \& PAINT THINNER } \\
& \text { UN: - MATERIAL UNYNOWN } \\
& \text { * - POSSIBLE OJL CONTAHINATION } \\
& \text { BK6 - BACKGROUNI SITE }
\end{aligned}
$$


FIGURE 35

GASOLINE RANGE HYDROCARBON

GHROMATOGRAM FOR SITE OT-25

$O T-25$
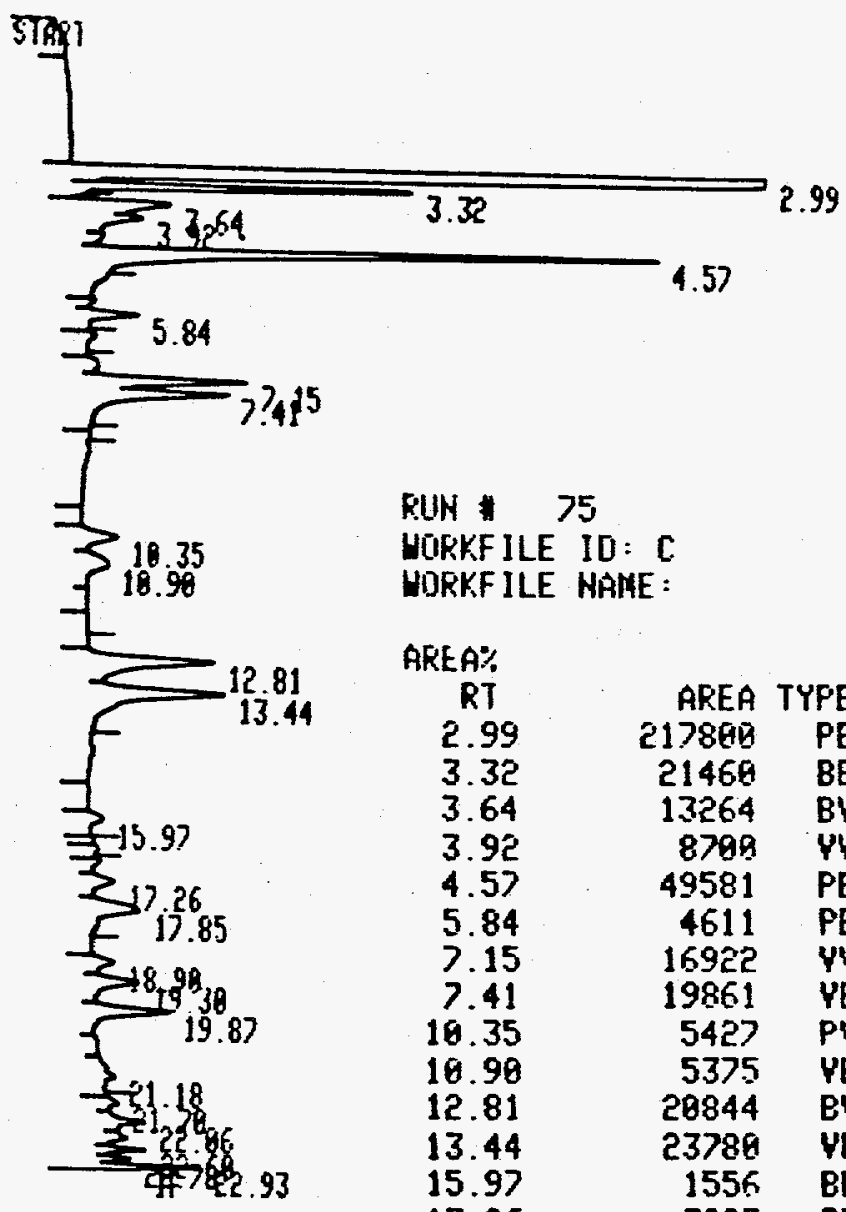

FUN 75

0CT/Q7/85 15:22:14

WORKF ILE 10: $\mathrm{C}$

MDRKF ILE NAIIE:

AREA\%

RT

2.99

3.32

3.64

3.92

4.57

5.84

7.15

7.41

18.35

10.98

12.81

13.44

15.97

17.26

17.85

18.96

19.36

19.87

21. 18

21.76

22.86

22.68

22.78

22.93

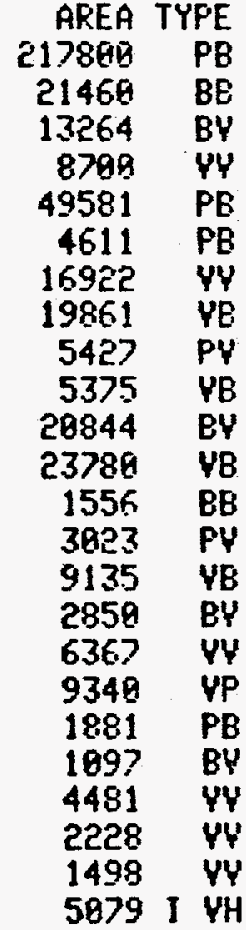

AR/HT

B. 144

o. 699

a. 265

e. 269

b. 131

0. 133

0.157

g. 297

o. 226

0.338

0.243

0. 259

0. 170

8. 282

6. 281

0.191

0.260

0.175

6. 268

0. 121

g. 165

8.080

ด. 082

0. 879

AREA\%

47.746.

4.785

c. 988

1.967

10. 869

1. 011

3.718

4.354

1.190

1.178

4.576

5.213

0. 341

0. 663

2. 003

0. 625

1. 396

2. 048

0.412

0.241

8.982

0. 486

0. 328

1. 113

IOTAL AREA=

456168

MUL FACTOK $=1.8089 E+B B$ 
FIGURE 36

GASOLINE RANGE HYDROCARBON

CHROMATOGRAM FOR SITE OT-29-

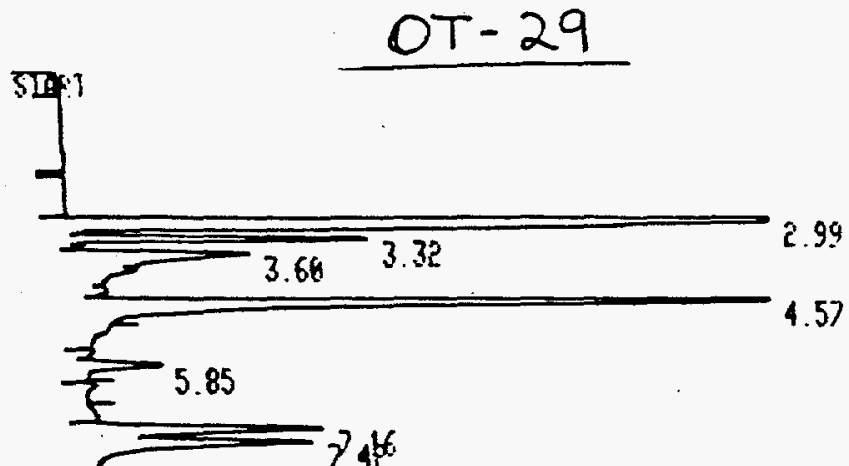

7
1
1
1

BUN 79

UIIKKFILE ID: C

0CT/87/86. 18:36:4y WUKKF ILE NAME:

AKt A\%

RI

2. .99

3.32

3.66

4.57

5.85

1.16

$1.4 \vec{c}$

1. 37

16. 82

12. $8 \mathrm{C}$

13.46

15.99

16.62

16.87

17.28

1).87

18.92

19.31

19.88

21.29

ह1). 71

207

2. $4 \hat{c}$

드. 61

نट. 79

टंट. 94

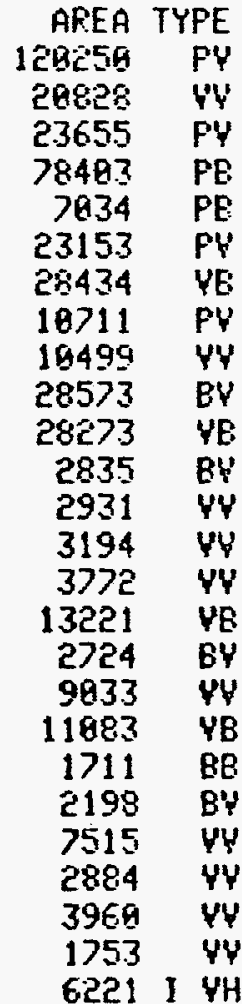

AF $>$ HT

0. 124

Q. 183

B. 199

घ. 125

0.134

๑. 159

4. 192

9. 224

6. 364

- .229

-. 239

Q. 199

d. 559

0.197

a. 218

G. 254

o. 189

6. 264

0.173

8. 295

ย. 157

0. 184

o. 115

0.057

อ. 891

0. $8 \% 8$
AKEA\%

¿'. $43 \%$

4.579

5. 2 . 1

17. 237

$1.54 \%$

5. $99 \mathrm{H}$

6. 251

2. 355

2. 348

6. ิ่bé

6.21t.

ด. $6 \mathrm{C}^{3}$

0.644

0.702

0. $8 \mathrm{e}^{\circ}$

2. 9 मे?

b. 599

1.986

2.437

0.376

0.435

1. $65 \mathrm{~F}$

B. 634

0.871

6. 385

1. 368

IUTAL AKEA $=\quad 454858$

MUL FACTUK $=1.8969 E+B 8$ 
FIGURE 37

GASOLINE RANGE HYDROCARBON

CHROMATOGRAM FOR SITE OT-58-
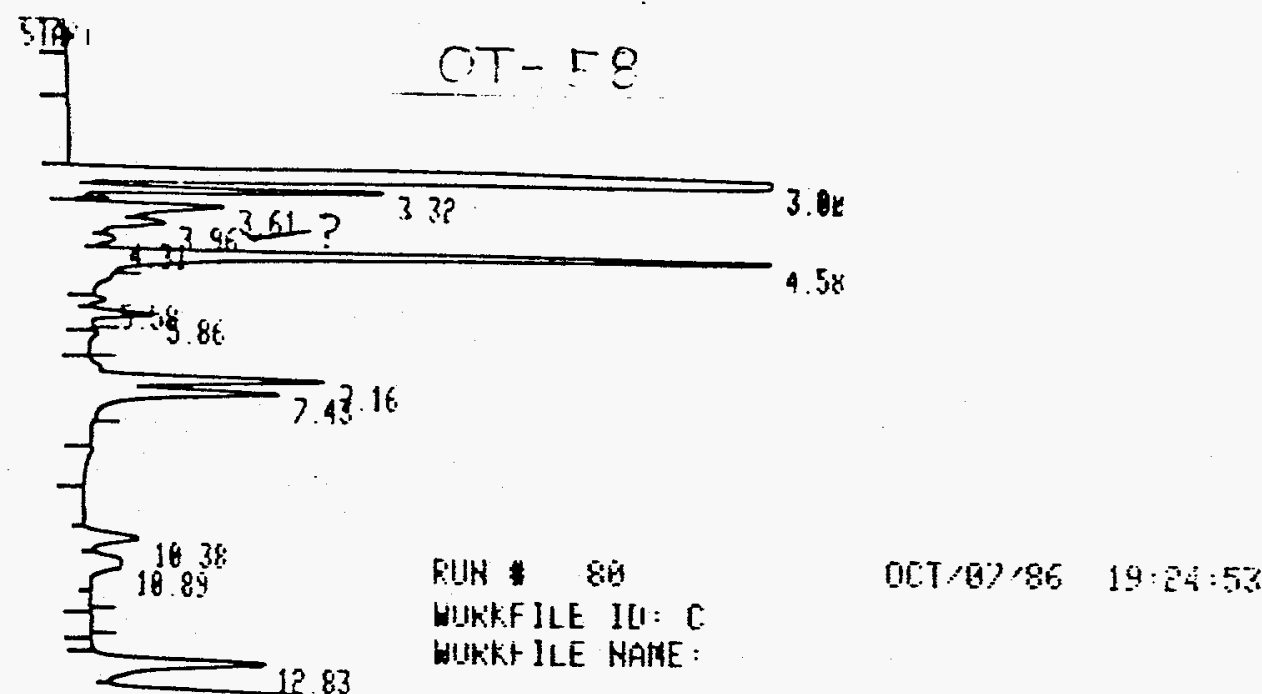

1

AREA\%

KI

$36 \overline{1}$

3.08

$\therefore 3 t$

3.61

3.96

4. 31

4.58

3.58

3.86

1.16

1.43

10.38

IV. 89

1‥ 83

13.46

15.99

16.63

$1 / .28$

17.88

18.92

19.31

19.88

21.20

21. 71

22.67

टc. 39

20.6é

$2 \cdot .79$

टर. 94

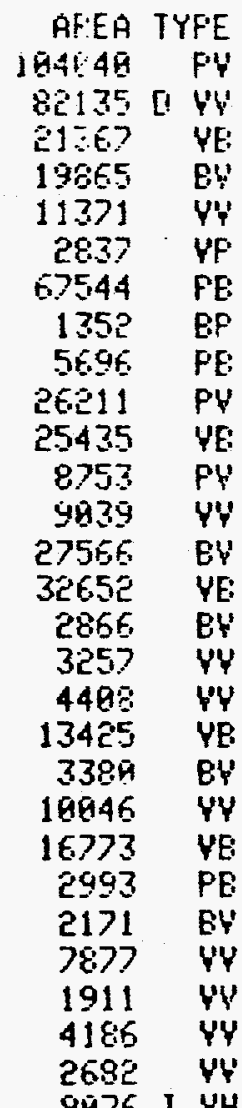

AK. HT

a. 981

AKEA\%

G. $954 \quad 15.470$

घ. $184 \quad 4.925$

ต. 199

6. 209

Q. 194

3.742

2. $14 \ddot{c}$

G. 124

Q. 125

0.534

12. $7 \mathrm{CC}^{\circ}$

B. 132

Q. 255

1.978

Q. 1.4

$4.93 \%$

0.199

4.731

4. 231

1.649

9. 367

1.785

ต. 234

5.192

G.231

0.199

6.154

$0.54 \mathrm{H}$

อ. 614

$0.83 \theta$

ด. 221

2. $52 y$

a. 254

6.63)

B. 195

1. $8 \mathrm{yi}^{\circ}$

0.199

3.159

0.169

(4. 50.4

o. 144

0.499

0.180

1.484

B. Q857

ช. 360

ค. 789

-. 585

$9 \dot{1}>6$ I YH

0.091

6.678

1. $71 \mathrm{H}$ 


\section{The Fire Training Area}

At the Fire Training Area a total of fifty-one sites were sampled for $\mathrm{C}_{1}-\mathrm{C}_{4}$ hydrocarbon soil gas analysis and seventeen sites were sampled for gasoline range hydrocarbons.

We were told that probably fuel oil and/or kerosene had been dumped in an area surrounded by a small burm. The oils were subsequently burned during fire training exercises. The area has not been used in several years, possibly since 1981 . The burm and surrounding area has been graded and today is fairly level with grass over most of the area. There was an area about $40^{\prime} \times 40^{\prime}$ where the grass appeared sparse and the ground disturbed. It was assumed that the perimeter of this area was the approximate location of the burm. In the northern corner of this area was an area of oil soaked soil.

The sample locations, shown in Figure 38, consist of a $6 \times 6$ site grid on $20 \mathrm{ft}$ centers, with other sites to fill in the grid and to test more distant areas. At Site 15, soil gas samples were taken at 1,2 and $3 \mathrm{ft}$. The maximum soil gas magnitudes were found at $3 \mathrm{ft}$, thus all other soil gas samples were taken at that depth in the Fire Training Area.

The soil gas data is shown in Figures $39-41$, and is tabulated in Tables 18 and 19. Clearly the largest soil gas contamination is associated with the fire training pit, with some movement to the south and west. This "displaced" contamination may have 
resulted from the movement of soil when the site was leveled. As was the case at the 0il Test Site, the soil gas hydrocarbons at large magnitude sites are mainly composed of methane, ethylene and propylene. These are most probably not primary contamination, but rather result from increased biological activity in oil soaked (organic rich) areas.

At seventeen sites, indicated on Figure 38, soil samples were taken for gasoline range hydrocarbons. As recorded in Table 20, hydrocarbons among the group sought were found at three of these sites, as shown in Figure 42. The largest amounts were found at Site 47, where a depth profile revealed maximum levels at a depth of $2 \mathrm{ft}$. The hydrocarbon found in greatest concentration was toluene at a level of $59.5 \mathrm{ppb}$.

The chromatographic trace at Site 47 is shown in Figure 43. In addition to the gasoline range hydrocarbons determined in this survey, it is obvious that many other compounds are present. As mentioned before, their identity could be ascertained using gas chromatographic-mass spectral techniques. 

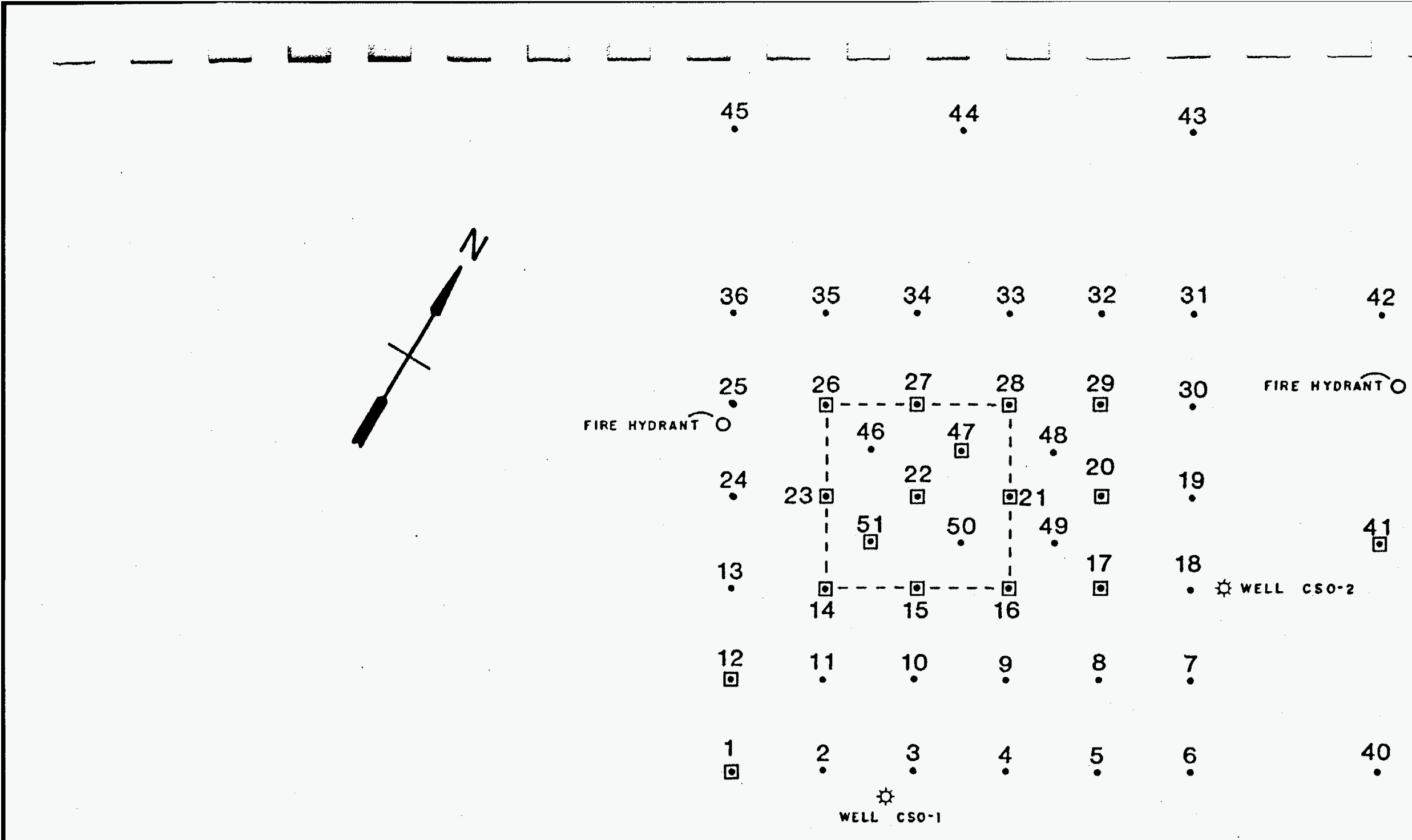

$0 \quad 1020 \mathrm{ft}$.

37

38

39

- soil gas samples

0 SOIL GAS \& SOIL SAMPLES

PROBABLE LOCATION OF OIL PIT

FIRE TRAINING AREA SITE LOCATION MAP 



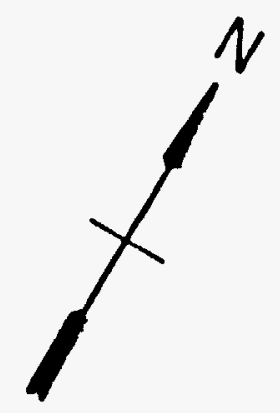

\section{LEGEND}

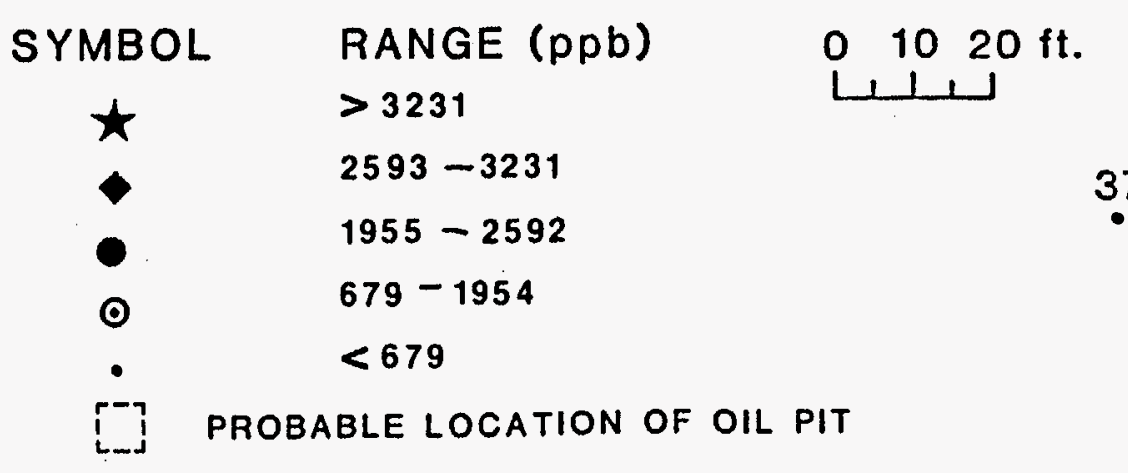

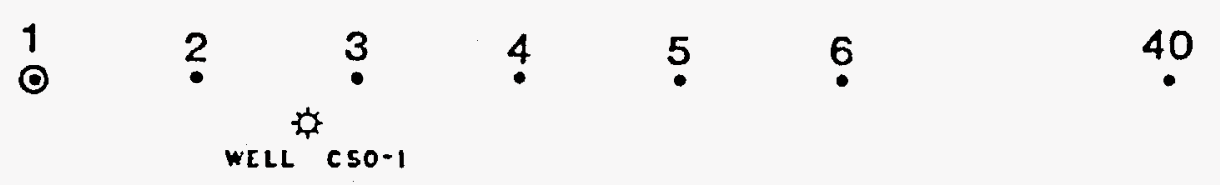





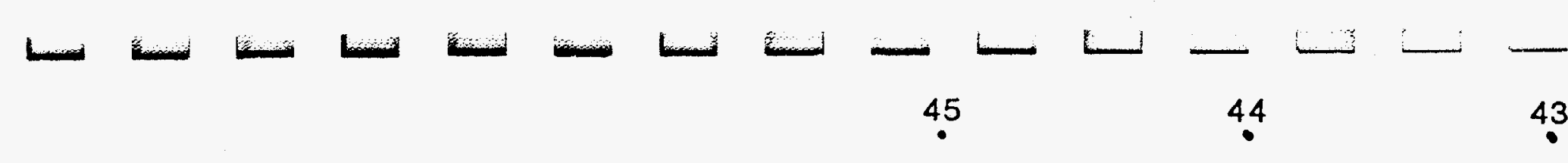
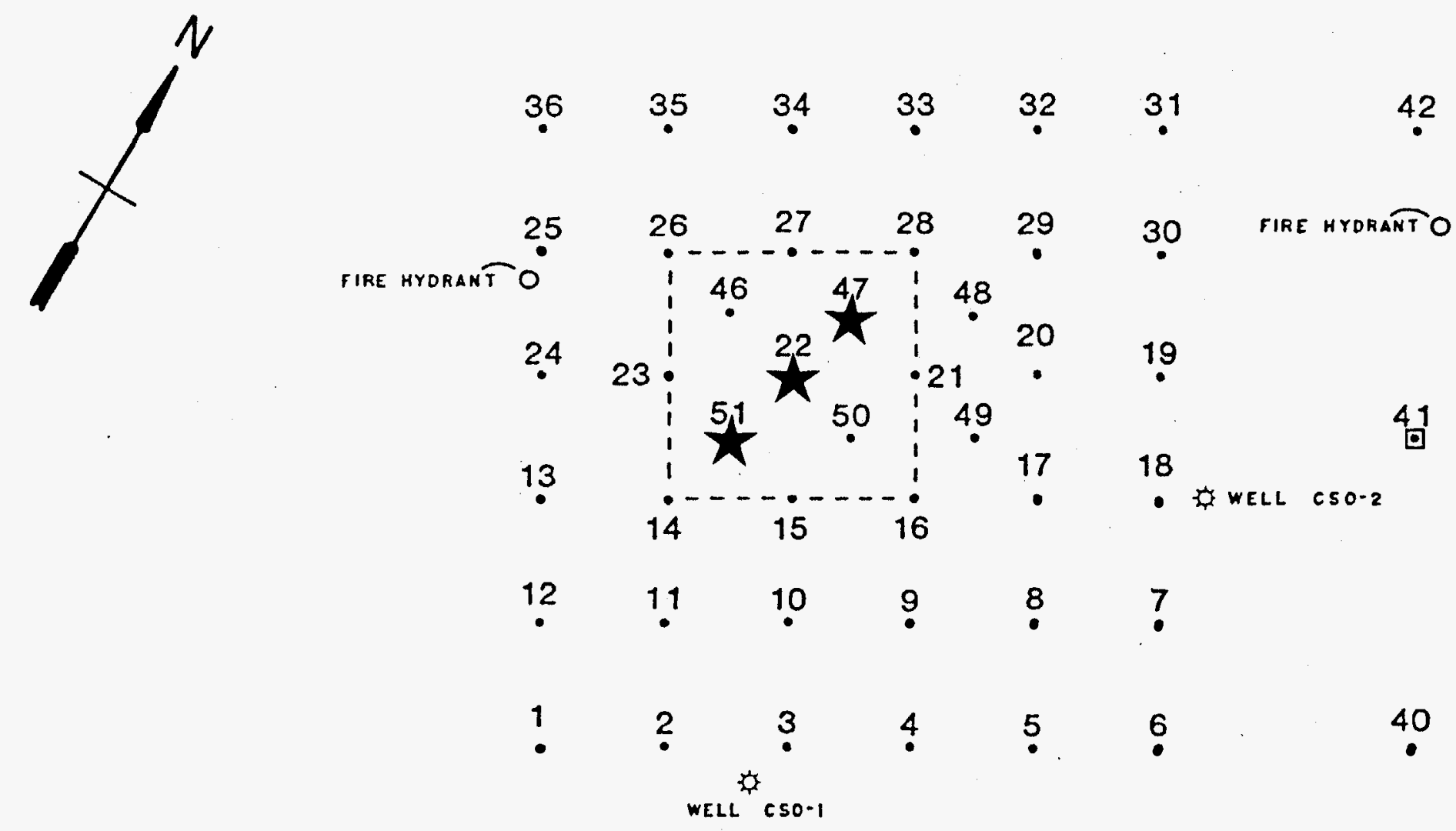

$0 \quad 1020 \mathrm{ft}$.

39

FIRE TRAINING AREA GASOLINE RANGE (C5-C8) NOVEMBER, 1986

S.R.P.

† compounds detected 
TABLE 18

E. I. DUPONT DE MEMOURS CO. INC., S.R.P., AIKEN, S.C. -...

---- FIRE TRAINING AREA -- PROBE SURVEY, OCT. 1986 -.--

--- SOIL GAS CONCENTRATIONS -..-

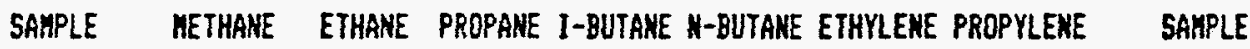

$\begin{array}{llllllll}1 & P P B & P P B & \text { PPB } & \text { PPB PPB } & \text { PPB } & \text { PPB }\end{array}$

\begin{tabular}{|c|c|c|c|c|c|c|c|c|c|c|}
\hline $\mathrm{FT}$ & 1 & 20714 & 706 & 210 & 243 & 86 & 411 & 129 & FT & 1 \\
\hline $\mathrm{FT}$ & 2 & 6003 & 310 & 99 & 169 & 40 & 210 & 222 & FT & 2 \\
\hline FT & 3 & 4078 & 224 & 76 & 141 & 34 & 159 & 176 & FT & 3 \\
\hline $\mathrm{FT}$ & 4 & 2458 & 118 & 39 & 71 & - & 83 & 82 & $\mathrm{FT}$ & 4 \\
\hline FT & 5 & 1843 & 45 & 15 & 29 & - & 27 & 30 & FT & 5 \\
\hline $\mathrm{FI}$ & 6 & 1366 & 79 & 29 & 62 & - & 64 & 77 & FT & 6 \\
\hline FT & 7 & 924 & 23 & 9 & 23 & - & 20 & 22 & FT & 7 \\
\hline FI & 8 & 1113 & 30 & 11 & 31 & - & 23 & 25 & FT & $B$ \\
\hline FT & 9 & 1233 & 167 & 63 & 41 & $2 b$ & 114 & 124 & FT & 9 \\
\hline FI & 10 & 4024 & 224 & 73 & 148 & 40 & 168 & 177 & $\mathrm{FI}$ & 10 \\
\hline FT & 11 & 1642 & 124 & 44 & 50 & 13 & 91 & 107 & $\mathbf{F T}$ & 11 \\
\hline FT & 12 & 16401 & 363 & 89 & 240 & 40 & 234 & 218 & FT & 12 \\
\hline FI & 13 & 2908 & 301 & 108 & 175 & 12 & 216 & 239 & $\mathrm{FT}$ & 13 \\
\hline FT & 14 & 9347 & 714 & 246 & 246 & 96 & 534 & 557 & FT & 14 \\
\hline $\mathrm{FT}$ & 15 & 47505 & 502 & 126 & 53 & 75 & 424 & 429 & FT & 15 \\
\hline FT & $15-1$ & 3398 & 94 & 32 & 4 & - & 150 & 98 & FT & $15-1$ \\
\hline$F T$ & $15-2$ & 15520 & 228 & 64 & 13 & - & 183 & 143 & FT & $15-2$ \\
\hline FI & 16 & 1593 & 123 & 43 & 76 & - & 95 & 102 & $\mathbf{F T}$ & 16 \\
\hline FI & 17 & 698 & 27 & 10 & 23 & - & 22 & 24 & $\mathrm{FT}$ & 17 \\
\hline FT & 18 & 1316 & 58 & 21 & 38 & - & 41 & 45 & FT & 18 \\
\hline FI & 19 & 2675 & 160 & 55 & 73 & 14 & 107 & 121 & FI & 19 \\
\hline FI & 20 & 869 & 30 & 11 & 18 & - & 22 & 25 & FT & 20 \\
\hline $\mathrm{FT}$ & 21 & 5479 & 497 & 170 & 182 & 65 & 331 & 348 & FI & 21 \\
\hline FI & 22 & 302630 & 482 & 147 & 108 & 113 & 567 & 722 & FI & 22 \\
\hline FT & 23 & 1170 & 142 & 57 & 18 & 19 & 81 & 87 & FT & 23 \\
\hline $\mathrm{FT}$ & 24 & 7100 & 913 & 305 & 203 & 108 & 514 & 539 & FT & 24 \\
\hline FT & 25 & 2049 & 55 & 25 & 24 & - & 42 & 54 & FI & 25 \\
\hline FT & 26 & 2781 & 365 & 144 & 70 & 60 & 206 & 247 & FT & 26 \\
\hline FT & 27 & 25864 & 3466 & 1029 & 187 & 331 & 1441 & 1442 & $\mathrm{FT}$ & 27 \\
\hline $\mathrm{FT}$ & 28 & 5791 & 622 & 190 & 30 & 59 & 304 & 270 & FT & 28 \\
\hline $\mathrm{FI}$ & 29 & 990 & 98 & 35 & 20 & - & 56 & 59 & FT & 29 \\
\hline FT & 30 & 2575 & 327 & 136 & 123 & 58 & 248 & 293 & FT & 30 \\
\hline FT & 31 & 1916 & 153 & 53 & 84 & 27 & 137 & 148 & FT & 31 \\
\hline FT & 32 & 1419 & 60 & 21 & 39 & - & 45 & 48 & FT & 32 \\
\hline FT & 33 & 2030 & 116 & 43 & 39 & - & 93 & 97 & FT & 33 \\
\hline FT & 34 & 4471 & 259 & 103 & 118 & 51 & 194 & 204 & FT & 34 \\
\hline FT & 35 & 2741 & 209 & 72 & 68 & 27 & 159 & 175 & FT & 35 \\
\hline FT & 36 & 10158 & 698 & 245 & 433 & 101 & 666 & 643 & FT & 36 \\
\hline FT & 37 & 8561 & 356 & 105 & 150 & 40 & 190 & 207 & FT & 37 \\
\hline FI & 38 & 1096 & 53 & 16 & 22 & - & 31 & 34 & FT & 38 \\
\hline FT & 39 & 1652 & 64 & 21 & 33 & - & 12 & 45 & FT & 39 \\
\hline FT & 40 & 14301 & 199 & 43 & 20 & - & 55 & 61 & FT & 40 \\
\hline FT & 41 & 514 & 37 & 11 & - & - & 28 & 29 & FT & 41 \\
\hline FT & 12 & 1877 & 83 & 25 & 22 & - & 53 & 53 & FT & 42 \\
\hline FT & 43 & 731 & 48 & 17 & - & - & 32 & 36 & FT & 43 \\
\hline FT & 44 & 5517 & 162 & 45 & 43 & - & $B 9$ & 96 & $F T$ & 44 \\
\hline FT & 45 & 6673 & 391 & 128 & 170 & 49 & 288 & 311 & FI & 45 \\
\hline FT & 46 & 306540 & 1358 & 398 & 130 & 137 & 2267 & 1778 & $\mathbf{F T}$ & 46 \\
\hline FT & 47 & 6131601 & 1190 & 231 & 449 & 569 & 3994 & 3841 & FT & 47 \\
\hline
\end{tabular}


TABLE 18 (cont)

E. 1. DUPONT DE NENOURS CO. INC., S.R.P., AIKEN, S.C. ---

--- fIRE TRAINING AREA -- PROBE SURVEY, OCT. 1986 -..-

-- SOIL GAS CONCENTRATIONS --..

\begin{tabular}{|c|c|c|c|c|c|c|c|c|c|}
\hline $\begin{array}{c}\text { SAIPLE } \\
1\end{array}$ & $\begin{array}{r}\text { METHANE } \\
\text { PPB }\end{array}$ & $\begin{array}{r}\text { ETHANE } \\
\text { PPB }\end{array}$ & $\begin{array}{r}\text { PROFANE } \\
\text { PPB }\end{array}$ & $\begin{array}{r}\text { I-BUTANE } \\
\text { PPB }\end{array}$ & $\begin{array}{r}\text { N-BUTANE } \\
\text { PPB }\end{array}$ & $\begin{array}{r}\text { ETHYLENE } \\
\text { PPB }\end{array}$ & $\begin{array}{c}\text { PROPYLENE } \\
\text { PPB }\end{array}$ & & PLE \\
\hline FT 48 & 887 & 43 & 17 & - & - & 33 & 31 & $\mathbf{F T}$ & 48 \\
\hline FT 49 & 14427 & 290 & 57 & 51 & - & 127 & 94 & FT & 49 \\
\hline FT 50 & 1025400 & 1096 & 290 & 192 & 322 & 1821 & 1687 & $F T$ & 50 \\
\hline FT 51 & 228500 & 396 & 113 & 87 & 81 & 658 & 608 & $F T$ & 51 \\
\hline
\end{tabular}

MDTE:

FT $\chi X$ - 3 FT. SAMPLE

FT $X X-1$ - 1 FT. SAKPLE

FT XXY-2 - 2 FT. SAMPLE 
TABLE 19

--- E. 1. DUPONT DE MEROURS CO. INC., S.R.P., AIKEN, S.C. ---

--- FIRE TRAINING AREA - PRDBE SURVEY, OCT. 1986 ----

--- SOIL GAS PERCENTAGES --.-

Sample total methane ethane propane butane

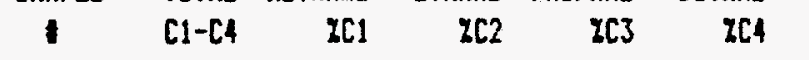

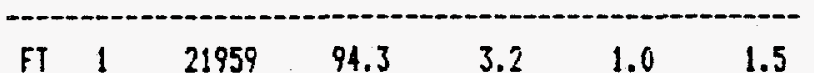

FT 2 - 6621

90.7

$\begin{array}{lll}4.7 & 1.5 & 3.2\end{array}$

FT 34553

FI 42686

FT $5 \quad 1932$

FT $6 \quad 1536$

FT 7979

FT $8 \quad 1185$

FT $9 \quad 1530$

FT $10 \quad 4509$

FT 11.1873

FI $12 \quad 17133$

FT $13 \quad 3534$

FT $14 \quad 10649$

FT 1548261

FT $15-1 \quad 3585$

FT $15-2 \quad 15855$

FT $16 \quad 1835$

FT $17 \quad 758$

FT $18 \quad 1433$

FT $19 \quad 2997$

FT $20 \quad 928$

FT $21 \quad 6393$

FT $22 \quad 303480$

FT $23 \quad 1406$

FT $24 \quad 8629$

FT $25 \quad 2153$

FT $26 \quad 3420$

FT $27 \quad 30877$

FT $28 \quad 6692$

FT $29 \quad 1143$

FT $30 \quad 3219$

FT $31 \quad 5233$

FT $32 \quad 1539$

FT $33 \quad 2228$

FT 34.5002

FT $35 \quad 3117$

FT $36 \quad 11635$

FT $37 \quad 9212$

FT $38 \quad 1187$

FT $39 \quad 1770$

FT $40 \quad 14563$

FT $41 \quad 565$

FT $42 \quad 2007$

FT $43 \quad 796$

FT $44 \quad 5767$

FT $45 \quad 7411$

FT 46308563

FT $47 \quad 6134043$ $\begin{array}{llll}89.6 & 4.9 & 1.7 & 3.8\end{array}$

$\begin{array}{llll}91.5 & 4.1 & 1.5 & 2.6\end{array}$

$\begin{array}{llll}95.4 & 2.3 & 0.8 & 1.5\end{array}$

$88.9 \quad 5.1$

94.4

93.9

80.6

89.2

87.7

95.7

82.3

87.8

98.4

94.8

97.9

86.8

92.1

91.8

89.3

93.6

85.7

99.7

83.2

82.3

95.2

81.3

83.8

86.5

86.6

80.0

93.9

92.2

91.1

89.4

87.9

87.3

92.9

92.3

93.3

98.2

91.0

93.5

91.8

95.7

90.0

99.3

100.0

$$
2.5
$$

1.9

2.5

$$
11.9
$$

r.o

t.6

2.1

6.7

1.0

2.6

1.1

6.7

3.6

4.0

5.3

$0.9 \quad 2.3$

4.1

1.6

4.4

4.2

3.4

0.5

1.6

3.1

2.3

6.1

3.2

0.3

0.9

0.4

0.3

1.7

0.3

2.3

1.3

1.5

1.8

3.2

1.2

2.7

2.7
0.0

0.2

10.1

4.1

10.6

3.5

1.2

10.7

4.2

11.2

3.3

2.8

8.6

3.1

10.2

4.2

1.0

3.9

1.4

5.2

5.2

1.9

6.7

2.1

6.0

$3.9 \quad 1.1$

$4.5 \quad 1.3$

$3.6 \quad 1.2$

1.40 .3

$6.5 \quad 2.5$

4.1 $\quad 1.2$

$6.0 \quad 2.1$

2.8

5.3

0.8

1.7

0.1

0.0

0.1

0.0

3.0

2.7

3.6

1.9

3.9

0.1

2.6

3.6

1.1

3.8

1.7

1.3

1.7

5.6

2.1

2.5

1.8

3.4

3.0

4.6

2.1

1.9

1.9

0.1

0.0

1.1

0.7

3.0

0.1
$0.9 \quad 2.6$
TOTAL ETHYLENE PROPYLENE SAAPLE HYDCARB $z$ I

$\begin{array}{rllll}22829 & 1.9 & 1.9 & \text { FT } & 1 \\ 7053 & 3.0 & 3.1 & \text { FT } & 2 \\ 4888 & 3.3 & 3.6 & \text { FT } & 3 \\ 2851 & 2.9 & 2.9 & \text { FI } & 1 \\ 1989 & 1.4 & 1.5 & \text { FT } & 5 \\ 1677 & 3.8 & 4.6 & \text { FT } & 6 \\ 1021 & 2.0 & 2.2 & \text { FT } & 7 \\ 1233 & 1.9 & 2.0 & \text { FT } & 8 \\ 1768 & 6.4 & 7.0 & \text { FT } & 9 \\ 1854 & 3.5 & 3.6 & \text { FT } & 10 \\ 2071 & 4.1 & 5.2 & \text { FT } & 11 \\ 17585 & 1.3 & 1.2 & \text { FT } & 12 \\ 3989 & 5.4 & 6.0 & \text { FI } & 13 \\ 11740 & 4.5 & 4.7 & \text { FT } & 14 \\ 19114 & 0.9 & 0.9 & \text { FT } & 15 \\ 3833 & 3.9 & 2.6 & \text { FT } & 15-1 \\ 16181 & 1.1 & 0.9 & \text { FT } & 15-2\end{array}$

0.0

0.0 
1314-0ct-86 4

$$
-91-
$$

$$
\text { TABLE } 19 \text { (cont) }
$$

--- E. 1. DUPONT DE MEMOURS CO. INC., S.R.P., AIKEN, S.C. ----

--- FIRE TRAINING AREA -- PROBE SURVEY, OCT. 1986 ----

\begin{tabular}{|c|c|c|c|c|c|c|c|c|c|c|}
\hline SAMPLE & $\begin{array}{l}\text { TOTAL } \\
\text { C1-CA }\end{array}$ & $\begin{array}{r}\text { METHANE } \\
\text { ZCI }\end{array}$ & $\begin{array}{r}\text { ETHANE } \\
\text { XC2 }\end{array}$ & $\begin{array}{r}\text { PROPANE } \\
\text { ICJ }\end{array}$ & $\begin{array}{r}\text { BUTANE } \\
\text { 2CA }\end{array}$ & $\begin{array}{c}\text { TOTAL } \\
\text { HYDCARB }\end{array}$ & $\begin{array}{r}\text { ETHYLENE } \\
?\end{array}$ & $\begin{array}{c}\text { YLENE } \\
2\end{array}$ & SAH & \\
\hline FT 48 & 947 & 93.7 & 4.5 & 1.8 & 0.0 & 1011 & 3.3 & 3.1 & & 48 \\
\hline FT $\$ 9$ & 14825 & 97.3 & 2.0 & 0.1 & 0.3 & 15046 & 0.8 & 0.6 & FT & 49 \\
\hline FT 50 & 1027300 & 99.8 & 0.1 & 0.0 & 0.1 & 1030808 & 0.2 & 0.2 & FT & 50 \\
\hline FT 51 & 229177 & 99.7 & 0.2 & 0.0 & 0.1 & 230443 & 0.3 & 0.3 & FT & 51 \\
\hline
\end{tabular}

--- SOIL GAS PERCENTAGES --.-

NOTE:

FT $X X-3$ FT. SAMPLE

FT XXX-1 - 1 FT. SAMPLE

FT $X X-2-2$ FT. SAMPLE 
E. I. DUPONT DE KEKOURS CO. INC., S.R.P., AIKEK S.C. --FIRE TRAINING AREA -- SOIL SAMPLES -- OCT. 1986 ---- SOIL CONCENTRATIONS BY MEJGHT -.--

\begin{tabular}{|c|c|c|c|c|c|c|c|c|c|c|}
\hline $\begin{array}{l}\text { SAMPLE } \\
\text { WUMBER }\end{array}$ & DEPTH & $\begin{array}{r}\text { PENTANE } \\
\text { PPB }\end{array}$ & $\begin{array}{r}\text { HEXANE } \\
\text { PPB }\end{array}$ & $\begin{array}{r}\text { HEPTANE } \\
\text { PPE }\end{array}$ & $\begin{array}{r}\text { BEXIENE } \\
\text { PPB }\end{array}$ & $\begin{array}{r}\text { OCTAME } \\
\text { PPB }\end{array}$ & $\begin{array}{r}\text { TOLUENE } \\
\text { PPB }\end{array}$ & $\begin{array}{l}\text { TOTAL } \\
\text { AREA }\end{array}$ & $\begin{array}{l}\text { SAMPLE } \\
\text { NUMBER }\end{array}$ & DEPTH \\
\hline FT 1 & 1.0 & - & - & - & - & - & - & 5985 & FT 1 & 1.0 \\
\hline FT 1 & 2.0 & - & - & - & - & - & - & 6102 & FT 1 & 2.0 \\
\hline FT $\quad 1 R$ & 2.0 & - & - & - & - & - & - & 11205 & $F T \quad I R$ & 2.0 \\
\hline FT 1 & 3.0 & - & - & - & - & - & - & 10155 & FT 1 & 3.0 \\
\hline FT 1 & 1.0 & - & - & - & - & - & - & 6246 & FT 1 & 4.0 \\
\hline FT 1 & 5.0 & - & - & - & - & - & - & 14291 & FT 1 & 5.0 \\
\hline FI 12 & 2.0 & - & - & - & - & - & - & 10737 & FT 12 & 2.0 \\
\hline FT 14 & 2.0 & - & - & - & - & - & - & 13275 & FT 14 & 2.0 \\
\hline FT 15 & 2.0 & - & - & - & - & - & - & 29982 & FT 15 & 2.0 \\
\hline FT 16 & 2.0 & - & - & - & - & - & - & 11268 & FT 16 & 2.0 \\
\hline FT 17 & 2.0 & - & - & - & - & - & - & 17002 & FT 17 & 2.0 \\
\hline FT 20 & 2.0 & - & - & - & - & - & - & 17223 & FT 20 & 2.0 \\
\hline FT 21 & 2.0 & - & - & - & - & - & - & 20730 & FT 21 & 2.0 \\
\hline FT 22 & 2.0 & 2.4 & - & - & - & 7.5 & 9.5 & 681970 & FT 22 & 2.0 \\
\hline FT 23 & 2.0 & - & - & - & - & - & - & 26617 & FT 23 & 2.0 \\
\hline FT 26 & 2.0 & - & - & - & - & - & - & 34288 & FT 26 & 2.0 \\
\hline FT 27 & 2.0 & - & - & - & - & - & - & 14678 & FT 27 & 2.0 \\
\hline FT 28 & 2.0 & - & - & - & - & - & - & 16043 & FT 28 & 2.0 \\
\hline FT 29 & 2.0 & - & - & - & - & - & - & 44576 & FT 29 & 2.0 \\
\hline FI 41 & 1.0 & - & - & - & - & - & - & 7201 & FT 41 & 1.0 \\
\hline FT $\$ 1$ & 2.0 & - & - & - & - & - & - & 6982 & FT 11 & 2.0 \\
\hline FI 41 & 3.0 & - & - & - & - & - & - & 9416 & FT 41 & 3.0 \\
\hline FT 11 & 1.0 & - & - & - & - & - & - & 7822 & FT 41 & 4.0 \\
\hline FT 11 & 5.0 & - & - & - & - & - & - & 7688 & FT 41 & 5.0 \\
\hline FT 17 & 1.0 & - & - & 2.3 & 2.3 & 6.4 & 6.6 & 959610 & FT 47 & 1.0 \\
\hline FI 47 & 2.0 & - & - & 3.3 & 3.7 & 10.1 & 59.5 & 1303900 & FT 47 & 2.0 \\
\hline FI 17 & 3.0 & - & - & - & 1.9 & 6.0 & 19.5 & 497400 & FT 17 & 3.0 \\
\hline FT 17 & 4.0 & - & - & - & 2.3 & 3.7 & 3.9 & 398220 & FT 17 & 4.0 \\
\hline FT 1] & 5.0 & - & - & - & - & - & - & 88959 & FT 47 & 5.0 \\
\hline FI 51 & 1.0 & - & - & - & - & - & - & 97940 & FT 51 & 1.0 \\
\hline FT 51 & 2.0 & - & - & - & - & 2.5 & 5.4 & 438260 & FT 51 & 2.0 \\
\hline FT 51 & 3.0 & - & - & - & - & - & 2.6 & 173130 & FT 51 & 3.0 \\
\hline FT 51 & 4.0 & - & - & - & - & - & 2.5 & 138830 & FT 51 & 1.0 \\
\hline FT 51 & 5.0 & - & - & - & - & - & - & 21501 & FT 51 & 5.0 \\
\hline
\end{tabular}


FIGURE 43

FIRE TRAINING AREA GASOLINE RANGE HYDROCARBON CHROMATOGRAM FOR SITE FT-47

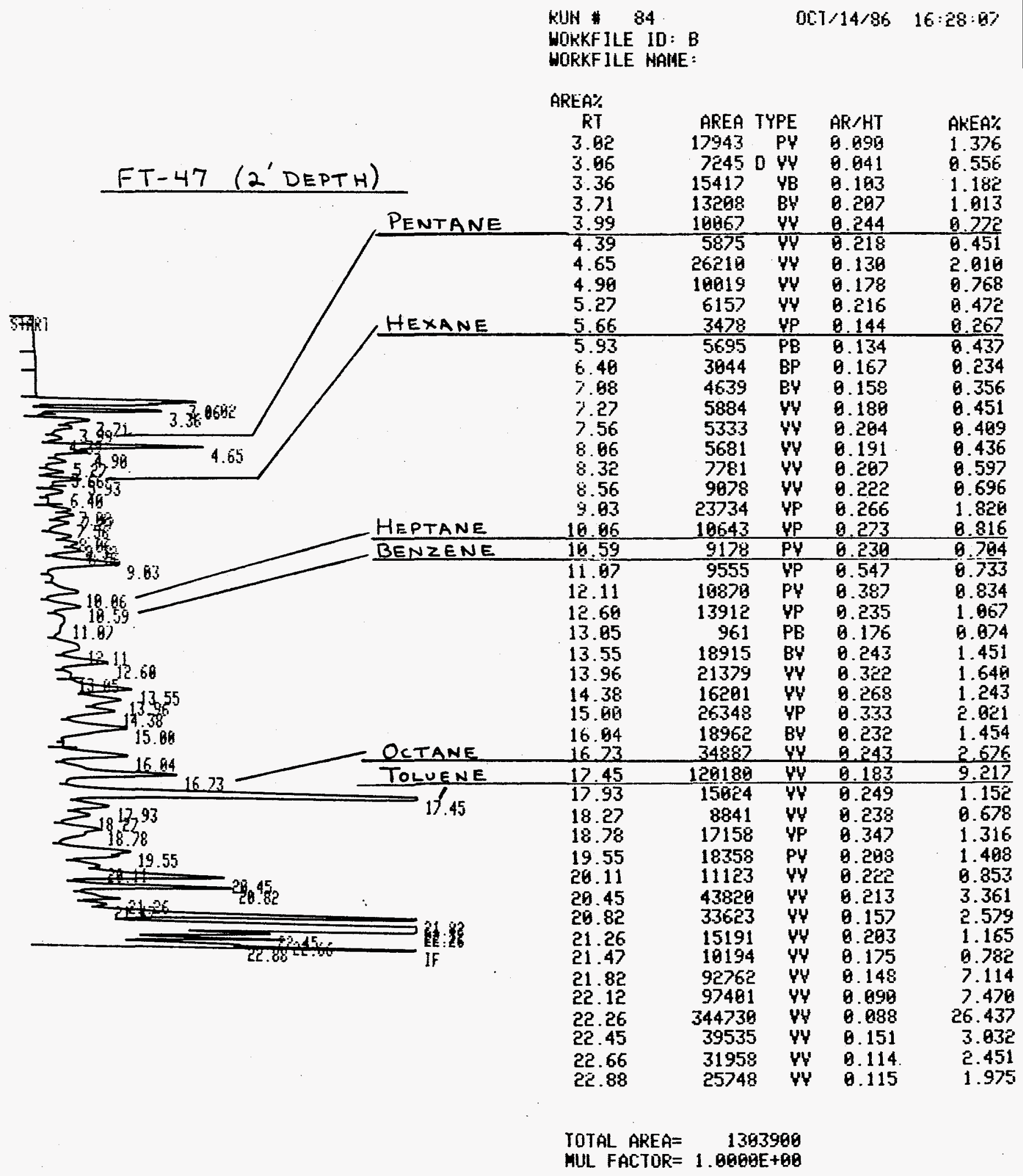


The Miscellaneous Chemicals Basin

At the Miscellaneous Chemicals Basin a total of 120 soil samples were taken for analysis of the halocarbons listed in Table 1. Of this total, seven sites were repeats of sites sampled in our prior survey conducted in the spring of 1986 under RFP85-43. In the earlier survey thirty-two sites were sampled. We have included those data in the statistics, maps and tables of this report. (Sites $1-32$ are the earlier survey; 'oct, 86

Sites 38-145 are the present survey.) In the case of repeat sites, only the data from the most recent survey was plotted on the maps, although both sets of data are 1isted in Table 22 .

Locations of all sites are shown on the Site Location Map in Figure 44. For the most part, the sites are located on $20 \mathrm{ft}$ centers and soil samples were taken from a depth of 18-24 inches.

The data (both sets) are shown in Table 21 , and are plotted in Figures 45-49. No data above minimum detection levels were found for vinyl chloride, 1,1-dichloroethane or 1,2-dichlorethane and only three sites were found which contained 1,1-dichloroethylene or methylene chloride. No maps were prepared for these compounds.

As may be determined from Table 22 and Figures 45 and 46, tetrachloroethylene and trichloroethylene have by far the largest magnitudes and are more widely distributed than the other halocarbons surveyed. Trichloromethane (chloroform), shown in. 
Figure 47, has considerably smaller magnitude, however, the higher samples cluster in the central portion of the basin. Only three sites had cis 1,2-dichloroethylene above minimum detection levels. These data are plotted in Figure 48. A number of sites, as shown in Figure 49, exhibited apparent trans 1,2-dichlorethylene levels above the minimum detection level. In both the earlier and present survey, we noticed that although the chromatographic peak for this compound had the proper retention time, the shape of the sample peak was different from that of the standard. We have also noticed that the range of the measurements, particularly for the present survey, is restricted. We therefore suggest caution in the interpretation of the data for this compound. 
E. 1. DUPONT DE MEMOURS CO. INC., S.R.P., AIKEN, SC. ----

MISCELLANEDUS CHEMICAL BASIN -- 1.5 FT. SOIL SAMPLES, OCTOBER 1983

---- SOIL COKCENTRATION BY MEIGHT IN PPB -..-

(satples 1 - 32 troe Jan. $86^{\prime}$ )

SAMPLE UIMYL 1,1-DiCl DiCl TI,2-DiCl 1,1-DiCl $[1,2-D i C l$ TriCl 1,2-DiCl TriCl TetraCl NUMBER CHLORJDE ETHYLENE METHANE ETHYLENE ETHANE ETHYLENE METHANE EthANE ETHYLENE ETHYLENE

\begin{tabular}{|c|c|c|c|c|c|c|c|c|c|c|c|}
\hline$p$ & 1 & $¥$ & - & - & 24.0 & - & 1 & 0.3 & t & 3.8 & 1.02 \\
\hline $\mathbf{p}$ & 2 & $\star$ & - & - & 21.0 & - & \pm & 1.9 & 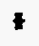 & 8.3 & 20.00 \\
\hline $\mathbf{P}$ & $2 R$ & - & - & - & - & - & - & 0.5 & - & 6.2 & 6.40 \\
\hline $\mathbf{P}$ & 3 & $*$ & - & - & 24.0 & - & \pm & 2.1 & $*$ & 6.8 & 296.00 \\
\hline$P$ & $3 R$ & - & - & - & - & - & 4.2 & 1.7 & - & 5.9 & 1200.00 \\
\hline $\mathbf{P}$ & 4 & $*$ & - & - & 18.0 & - & 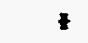 & - & 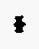 & 0.1 & 13.00 \\
\hline$P$ & $4 R$ & - & - & - & - & - & - & - & - & - & 0.10 \\
\hline $\mathbf{P}$ & 5 & 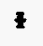 & - & - & 18.0 & - & 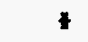 & - & t & - & 3.16 \\
\hline P & 6 & $¥$ & - & - & 24.0 & - & $¥$ & - & $\$$ & - & 1.96 \\
\hline$P$ & 7 & I & - & - & 21.0 & - & 1 & - & 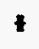 & - & 0.34 \\
\hline $\mathbf{P}$ & 8 & * & - & - & 24.0 & - & $*$ & - & $\$$ & - & 0.13 \\
\hline$P$ & 9 & $*$ & - & - & 24.0 & - & $¥$ & - & & - & - \\
\hline$P$ & 10 & $¥$ & - & - & 33.0 & - & * & - & $z$ & - & 0.04 \\
\hline $\mathbf{P}$ & 11 & \pm & - & - & 30.0 & - & $*$ & - & t & - & - \\
\hline$P$ & 12 & $*$ & - & - & 18.0 & - & * & - & $\mp$ & 0.1 & - \\
\hline $\mathrm{P}$ & 13 & * & - & - & 24.0 & - & $¥$ & 0.6 & 1 & 7.8 & 2.66 \\
\hline$P$ & $13 R$ & - & - & - & - & - & - & - & - & 0.1 & 0.10 \\
\hline$P$ & 14 & $\star$ & - & - & 42.0 & - & * & - & $z$ & 0.2 & 0.05 \\
\hline $\mathrm{P}$ & 15 & * & - & - & 39.0 & - & \pm & 0.1 & 1 & 2.6 & 0.29 \\
\hline$P$ & 16 & * & - & - & 24.0 & - & 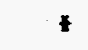 & 0.3 & $t$ & 1.7 & 1.24 \\
\hline$P$ & 17 & * & - & - & 42.0 & - & $*$ & 0.3 & 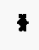 & 7.5 & 99.60 \\
\hline$P$ & 178 & - & - & - & 3.6 & - & - & - & - & 0.1 & 4.40 \\
\hline F & 18 & 1 & - & - & 48.0 & - & $¥$ & - & $z$ & 0.2 & 0.07 \\
\hline$P$ & 19 & * & - & - & 36.0 & - & $\star$ & - & $¥$ & - & - \\
\hline$P$ & 20 & $¥$ & - & - & 36.0 & - & 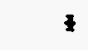 & - & 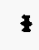 & 1.2 & - \\
\hline$P$ & 21 & 1 & - & - & 33.0 & - & 1 & - & $*$ & 0.2 & - \\
\hline$P$ & 22 & 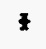 & - & - & 27.0 & - & \pm & - & \pm & 0.1 & - \\
\hline$P$ & 23 & 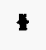 & - & - & 24.0 & - & 1 & - & 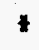 & - & - \\
\hline$P$ & 24 & 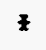 & - & - & 24.0 & - & \pm & 0.1 & $z$ & - & - \\
\hline$p$ & 25 & $i^{*}$ & - & - & 21.0 & - & 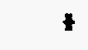 & - & 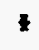 & - & - \\
\hline $\mathbf{P}$ & $2 b$ & $\mp$ & - & - & 2.0 & - & $z$ & 0.1 & 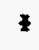 & - & 0.09 \\
\hline$P$ & 27 & ¥ & - & - & 4.0 & - & 1 & 0.1 & $z$ & 0.1 & 0.12 \\
\hline$P$ & 28 & \pm & - & - & 4.0 & - & $\mp$ & 0.1 & 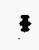 & 35.2 & 216.00 \\
\hline $\mathbf{P}$ & $28 R$ & - & - & 0.6 & 7.5 & - & 15.9 & 0.3 & - & 660.0 & 44800.00 \\
\hline P & 29 & * & - & - & 4.0 & - & 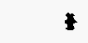 & - & $*$ & 0.1 & 3.22 \\
\hline $\mathbf{P}$ & $29 R$ & - & - & - & 3.3 & - & - & - & - & - & 4.30 \\
\hline $\mathrm{P}$ & 30 & 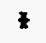 & - & - & 4.0 & - & $\star$ & - & \pm & 0.1 & 1.24 \\
\hline$P$ & 31 & $\star$ & - & - & 3.0 & - & \pm & 0.1 & 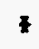 & 0.2 & 0.83 \\
\hline$P$ & 32 & $¥$ & - & - & 4.0 & - & t & - & $*$ & - & 0.41 \\
\hline$P$ & 33 & - & - & - & - & - & - & - & - & - & - \\
\hline$P$ & 34 & - & - & - & - & - & - & - & - & - & - \\
\hline$P$ & 35 & - & - & - & - & - & - & - & - & - & - \\
\hline$P$ & 36 & - & - & - & - & - & - & - & - & 0.1 & 560.00 \\
\hline$P$ & 37 & - & - & - & 7.2 & - & - & - & - & - & - \\
\hline$P$ & 38 & - & - & - & - & - & - & - & - & - & - \\
\hline$p$ & 39 & - & - & - & - & - & - & - & - & - & 0.12 \\
\hline $\mathrm{P}$ & 40 & - & - & - & - & - & - & 0.1 & - & 11.0 & 24.50 \\
\hline$P$ & 41 & - & - & - & - & - & - & - & - & - & 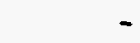 \\
\hline
\end{tabular}


--- E. I. DUPONT DE MEMOURS CO. INC., S.R.P., AIKEN, SC. ----

MISCELLANEOUS CHEMICAL BASIN -- 1.5 FT. SOIL SAMPLES, OCTOBER 1986

-..- SOIL CONCENTRATION BY WEIGHT IN PPB -...

(5anples 1 - 32 from Jan. 86\%)

\begin{tabular}{|c|c|c|c|c|c|c|c|c|c|c|c|}
\hline & $\begin{array}{l}\text { APLE } \\
\text { 1BER }\end{array}$ & $\begin{array}{r}\text { VINYL } \\
\text { CHLORIDE }\end{array}$ & $\begin{array}{l}1,1-D i C l \\
\text { ETHYLEHE }\end{array}$ & $\begin{array}{r}\text { DiCl } \\
\text { METHANE }\end{array}$ & $\begin{array}{l}T 1,2+D i C l \\
\text { ETHYUENE }\end{array}$ & $\begin{array}{r}1,1-\text { DiCl } \\
\text { ETHANE }\end{array}$ & $\begin{array}{r}C 1,2 \text { DICl } \\
\text { ETHYLENE }\end{array}$ & $\begin{array}{r}\text { TriCl } \\
\text { METHANE }\end{array}$ & $\begin{array}{r}\text { 1,2-DiCl } \\
\text { ETHANE }\end{array}$ & $\begin{array}{r}\text { Tricl } \\
\text { ETHYLENE }\end{array}$ & $\begin{array}{l}\text { Tetracl } \\
\text { ETHYLENE }\end{array}$ \\
\hline$p$ & 42 & - & - & - & - & - & t? & - & - & - & - \\
\hline $\mathbf{P}$ & 43 & - & - & - & - & - & - & - & - & - & - \\
\hline $\mathbf{P}$ & 44 & - & - & - & - & - & - & - & - & - & - \\
\hline$P$ & 45 & - & - & - & - & - & - & - & - & - & - \\
\hline$P$ & 46 & - & - & - & - & - & - & - & - & - & - \\
\hline$p$ & 47 & - & - & - & - & - & - & - & - & 1.9 & 1.60 \\
\hline$P$ & 48 & - & - & - & - & - & - & 0.1 & - & 0.1 & 0.80 \\
\hline$P$ & 49 & - & - & - & 3.0 & - & - & - & - & - & - \\
\hline P. & 50 & - & - & - & 3.7 & - & - & - & - & - & - \\
\hline $\mathbf{p}$ & 51 & - & - & - & - & - & - & - & - & - & - \\
\hline $\mathbf{P}$ & 52 & - & - & - & - & - & - & - & - & 0.1 & - \\
\hline P & 53 & - & - & - & - & - & - & - & - & 1.8 & 0.20 \\
\hline $\mathbf{P}$ & 54 & - & 0.7 & - & 3.9 & - & 295.0 & 13.2 & - & 5520.0 & 96.00 \\
\hline $\mathbf{P}$ & 55 & - & - & - & - & - & - & 0.4 & - & 23.0 & 9.50 \\
\hline $\mathbf{P}$ & 56 & - & - & - & 3.0 & - & - & - & - & 0.1 & - \\
\hline P & 57 & - & - & - & - & - & - & 1.4 & - & 7.8 & 44.00 \\
\hline $\mathbf{P}$ & 58 & - & 0.3 & - & - & - & - & 18.2 & - & 640.0 & 9333.00 \\
\hline $\mathbf{P}$ & 59 & - & - & - & - & - & - & - & - & - & 18.50 \\
\hline$p$ & 60 & - & - & - & - & - & - & 0.2 & - & 5.9 & 40.00 \\
\hline P & 61 & - & - & - & - & - & - & - & - & - & - \\
\hline p & 62 & - & - & - & - & - & - & - & - & - & 0.40 \\
\hline$P$ & 63 & - & - & - & - & - & - & - & - & - & - \\
\hline $\mathbf{P}$ & 64 & - & - & - & - & - & - & - & - & - & - \\
\hline $\mathrm{P}$ & 65 & - & - & - & - & - & - & - & - & - & - \\
\hline P & 66 & - & - & - & - & - & - & - & - & - & - \\
\hline $\mathbf{P}$ & 67 & - & - & - & - & - & - & - & - & - & - \\
\hline p & 68 & - & - & - & - & - & - & 0.3 & - & 0.3 & 15.30 \\
\hline$p$ & 69 & - & - & - & - & - & - & - & - & 0.1 & 0.80 \\
\hline$p$ & 70 & - & - & - & - & - & - & - & - & 0.2 & 0.09 \\
\hline P & 71 & - & - & - & - & - & - & 0.7 & - & 1.4 & 0.60 \\
\hline$P$ & 72 & - & - & - & - & - & - & - & - & - & - \\
\hline$P$ & 73 & - & - & - & - & - & - & - & - & 0.1 & - \\
\hline$P$ & 74 & - & - & - & - & - & - & - & - & 0.3 & 0.10 \\
\hline$P$ & 75 & - & - & - & 3.6 & - & - & - & - & 0.1 & 0.10 \\
\hline P & 76 & - & - & - & 4.4 & - & - & - & - & - & - \\
\hline $\mathbf{P}$ & 77 & - & - & - & 4.8 & - & - & - & - & - & 0.03 \\
\hline $\mathbf{P}$ & 78 & - & - & - & 8.0 & - & - & - & - & - & - \\
\hline $\mathbf{P}$ & 79 & - & - & - & - & - & - & - & - & - & 0.04 \\
\hline $\mathrm{P}$ & 80 & - & - & - & - & - & - & - & - & - & - \\
\hline $\mathbf{P}$ & 81 & - & - & - & 4.1 & - & $\begin{array}{l}- \\
-\end{array}$ & - & - & - & 0.04 \\
\hline 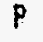 & 82 & - & - & - & 5.5 & - & $\begin{array}{l}- \\
-\end{array}$ & - & - & - & - \\
\hline $\mathbf{P}$ & 83 & - & - & - & 6.5 & - & - & 0.1 & - & 5.6 & 1640.00 \\
\hline $\mathbf{P}$ & 84 & - & - & - & - & - & - & 3.2 & - & 13.5 & 4800.00 \\
\hline $\mathrm{P}$ & 85 & - & - & - & 2.9 & - & - & - & - & - & 0.10 \\
\hline$P$ & 86 & - & - & - & - & - & - & - & - & - & - \\
\hline $\mathbf{p}$ & 87 & - & - & - & - & - & - & - & - & - & - \\
\hline$P$ & 88 & - & - & - & - & - & - & - & - & - & - \\
\hline $\mathbf{P}$ & 89 & - & - & - & - & - & - & - & - & - & - \\
\hline
\end{tabular}


E. 1. DUPONT DE NEMOURS CO. INC., S.R.P., AIKEN, SC. -..-

--- MISCELLANEOUS CHEMICAL BASIN -- 1.5 FT. SOIL SAMPLES, OCTOBER $1986---$

-- SOIL CONCEHTRATION BY HEIGHT IN PPB ----

(5amples 1 - 32 fron Jan. 8\%

S SAMPLE VINYL 1,1-DiCl DiCl T1,2-DiCl 1,1-DiCl C1,2-1iCl TriCl 1,2-DiCl TriCl TetraCl HuMEER CHLORIDE ETHYLENE METHANE ETHYLENE ETHANE ETHYLFNE METHANE ETHANE ETHYLENE ETHYLENE

P 90

p 91

P 92

P 93

$P 94$

P 95

p. 96

P 97

P 98

p 99

$P 100$

P 101

P 102

P 103

T $P 104$

P 106

P 107

1

P 108

P 109

P 110

| 111

P 112

$P 113$

3

P. 114

P 115

$P 116$

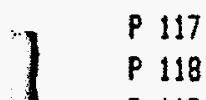

P 119

P 120

) $P 121$

P 122

$P 123$

$7 \quad P 124$

P 125

P 126

$P 127$
$P 128$

P 129

P 130

I P 131

P 132

P 133

P 134

P 135

P 136

P 137 
$-99-$

TABIE 21 (cont)

\#12-Dec-86 \$\$

MICROSEEPS LTD

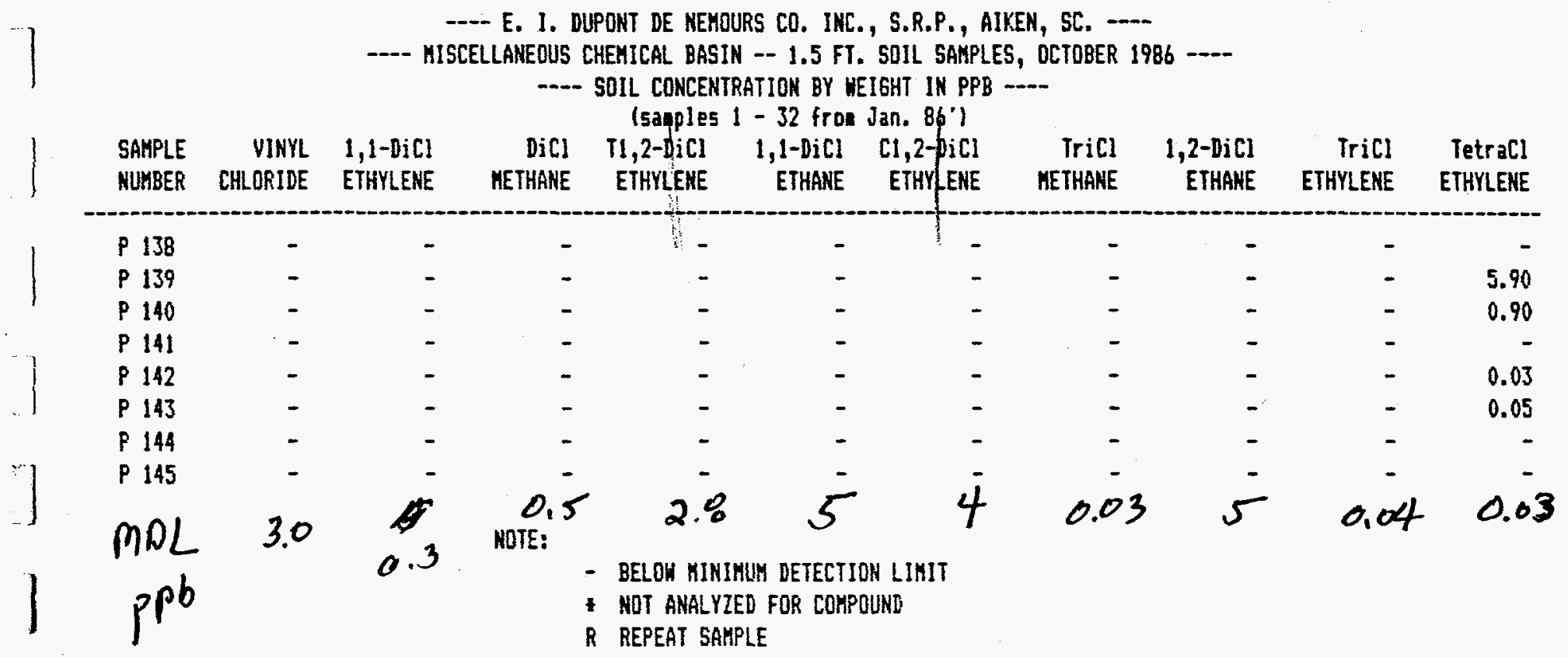

]

]

]

]

]

]

$$
\text { ] }
$$




\section{FIGURE 44}

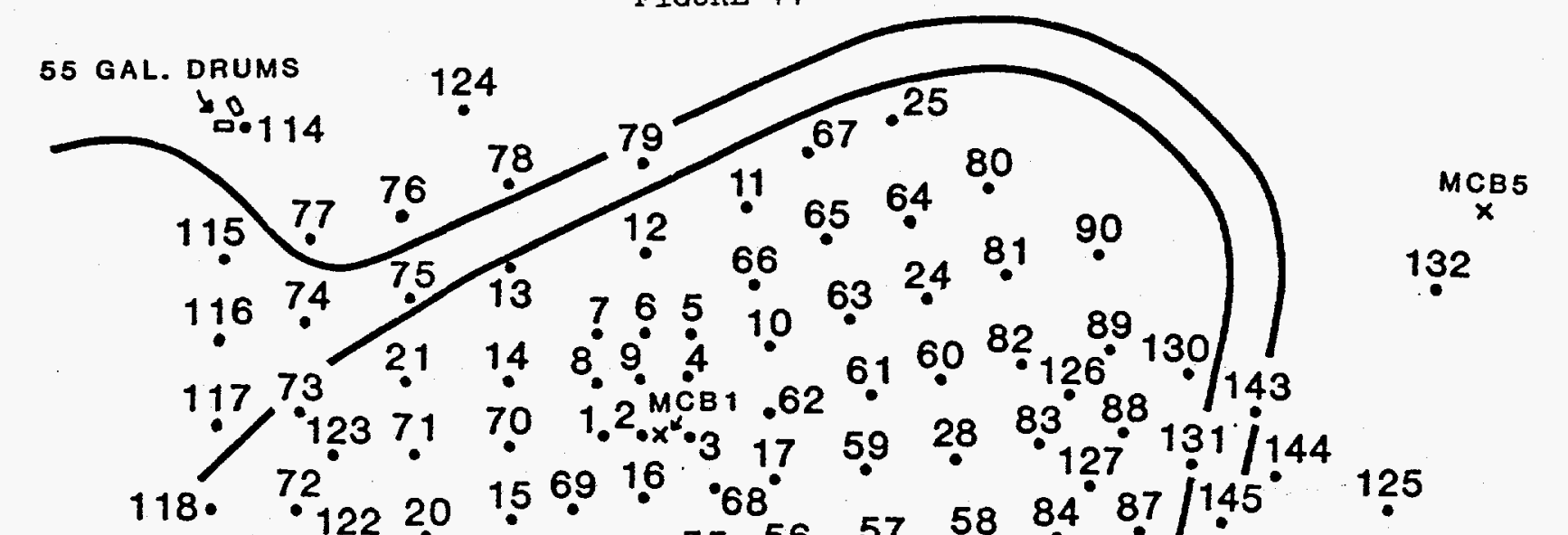

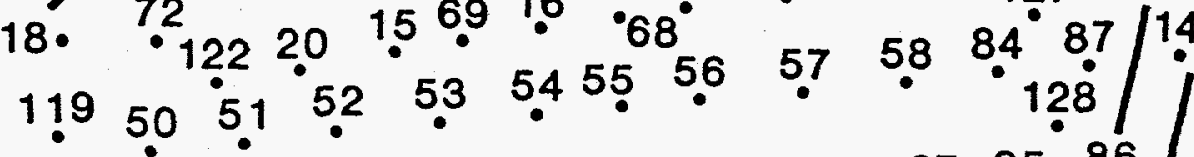
$\begin{array}{llllllllllll}120 & 22 & 46 & 19 & 47 & 18 & 48 & 26 & 49 & 27 & 85 & 86\end{array}$ $\begin{array}{lllllllllll}121 & 37 & 38 & 39 & 40 & 4.1 & 42 & 43 & 44 & 45 & 129\end{array}$ $\begin{array}{llllllllll}91 & 23 & 36 & 29 & 35 & 30 & 34 & 31 & 33 & 32\end{array}$ 92939495969798 10610510410310210110099

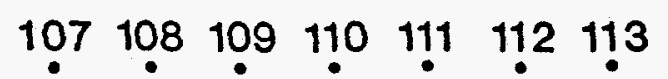

$\begin{array}{lllll}133 & 134 & 135 & 136 & 137\end{array}$

$142141 \quad 140139138$

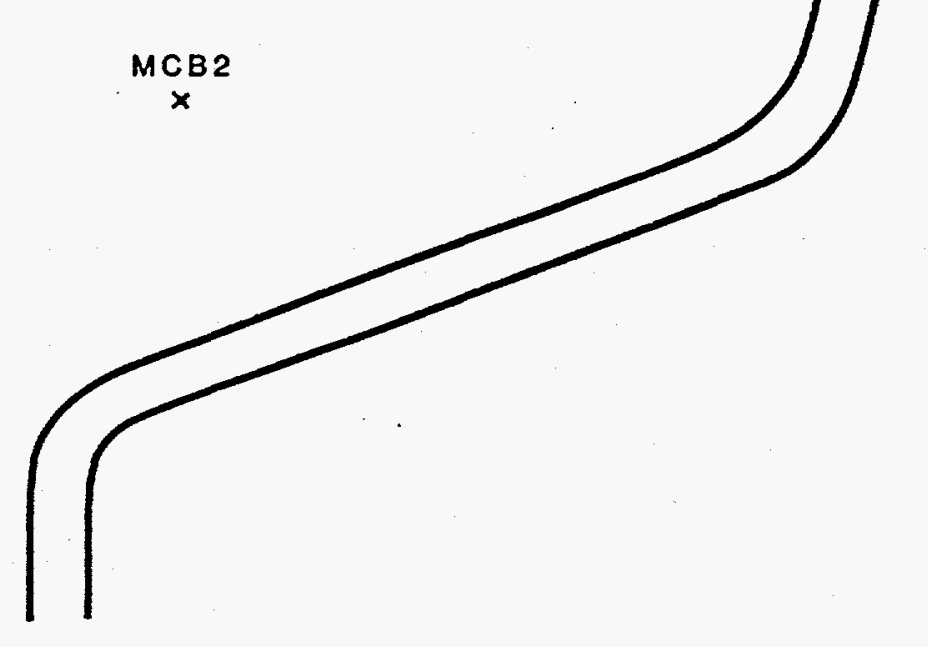

- SOIL SAMPLE LOCATION $x$ Proposed water wells

MISCELLANEOUS CHEMICAL BASIN SITE LOCATION MAP S.R.P. NOVEMBER, 1986 
FIGURE 45

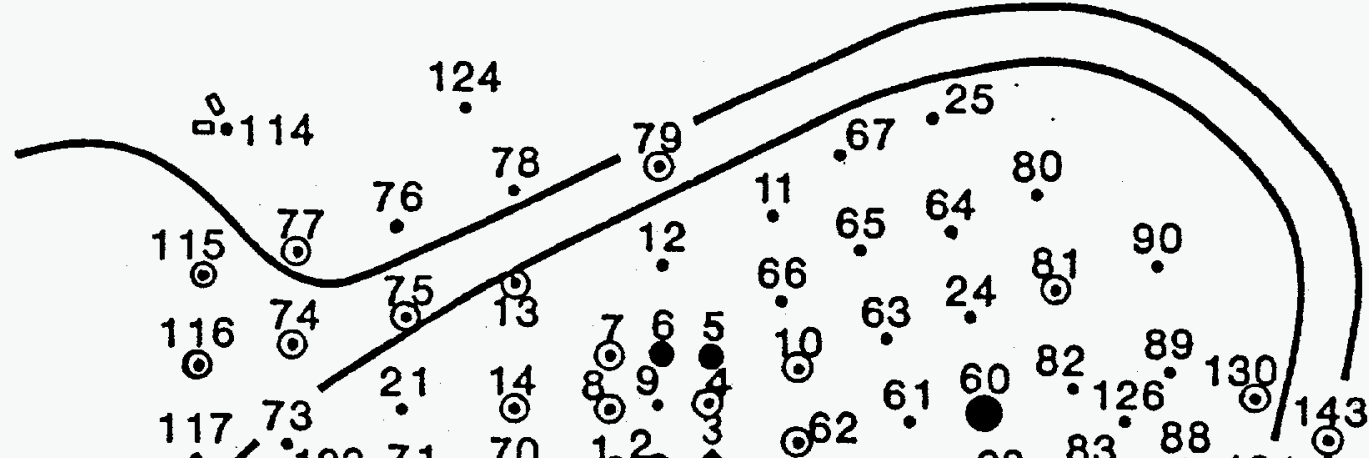

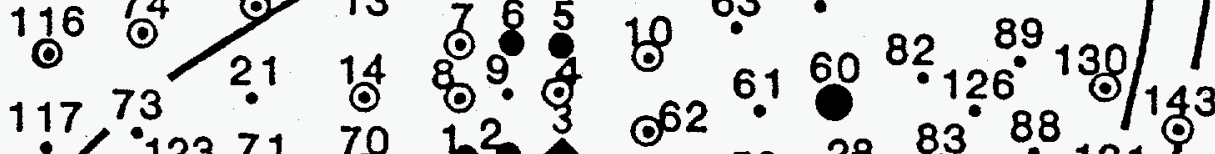

132

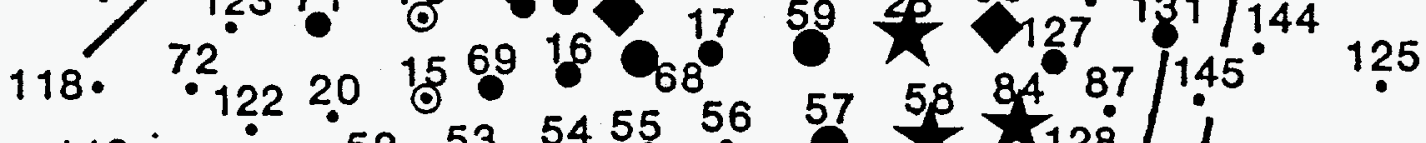

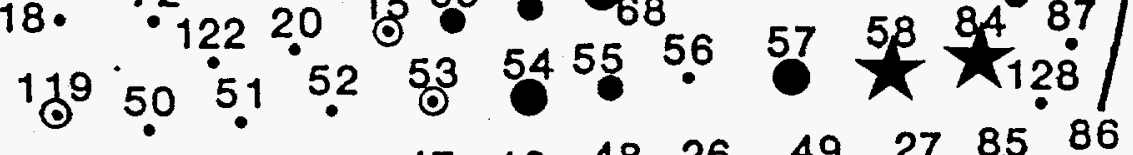
$\begin{array}{llllllllllll}120 & 22 & 46 & 19 & 47 & 18 & 48 & 26 & 49 & 27 & 85 & 86\end{array}$ $\begin{array}{lllllllllll}121 & 37 & 38 & 39 & 40 & 41 & 42 & 43 & 44 & 45 & 129\end{array}$ $\begin{array}{lllllllllllllllllllll}91 & 23 & 36 & 29 & 35 & 30 & 34 & 31 & 33 & 32\end{array}$ $9293 \quad 9495 \quad 969798$ 10610510410310210110099

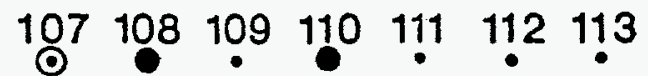

133134135136137

$142141 \quad 140139138$

]

1

]

LEGEND

נ

SYMBOL RANGE (ppb)

$\begin{array}{ll}\star & >4700 \\ & 500-4600 \\ & 11-100 \\ - & .6-10 \\ 0 & .03-.5 \\ - & \text { BELOW M.D.L. }\end{array}$

MISCELLANEOUS CHEMICAL BASIN

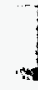




\section{FIGURE 46}

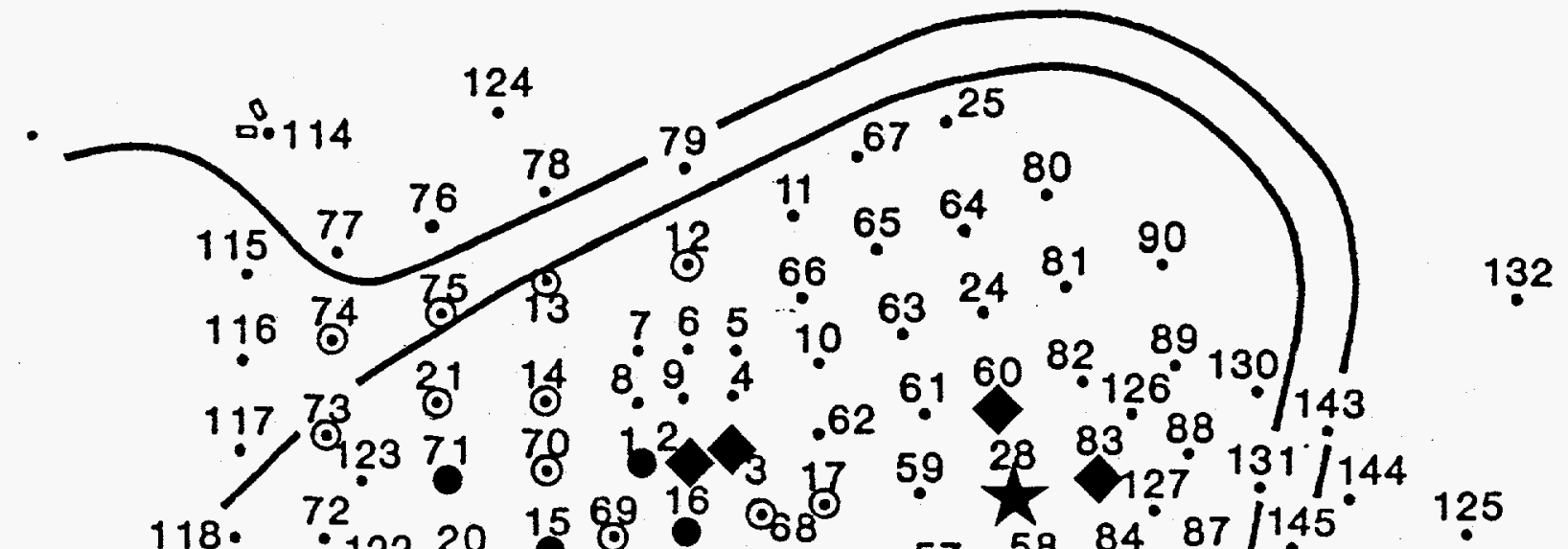
\begin{tabular}{lllllllll}
119 & 50 & 5 & 52 & 53 & 54 & 55 & 56 & 57 \\
\hline
\end{tabular} $\begin{array}{llllllllllll}120 & 22 & 46 & 19 & 47 & 18 & 48 & 26 & 49 & 27 & 8.5 & 86 \\ 0 & 0 & 0 & 0 & 0 & 0 & 0 & 0 & 0 & j\end{array}$ $\begin{array}{lllllllllll}121 & 37 & 38 & 39 & 40 & 4.1 & 42 & 43 & 44 & 45 & 129\end{array}$ $\begin{array}{llllllllll}91 & 23 & 36 & 29 & 35 & 38 & 34 & 81 & 33 & 32\end{array}$ $\begin{array}{llllllllll}92 & 93 & 94 & 95 & 96 & 97 & 98\end{array}$ 10610510410310218110099 $\begin{array}{lllllllll}107 & 108 & 109 & 110 & 111 & 112 & 113\end{array}$ 133134135136137

142141140139138

]

]

1

]

\section{LEGEND}

] $S Y M B O L$ RANGE (ppb)

了

$\begin{array}{ll}\star & 600-6000 \\ - & 5-25 \\ - & .5-4 \\ 0 & .1-.4 \\ - & \text { BELOW M.D.L. }\end{array}$

MISCELLANEOUS CHEMICAL BASIN

TRICHLOROETHYLENE

S.R.P.

NOVEMBER, 1986 


\section{FIGURE 47}

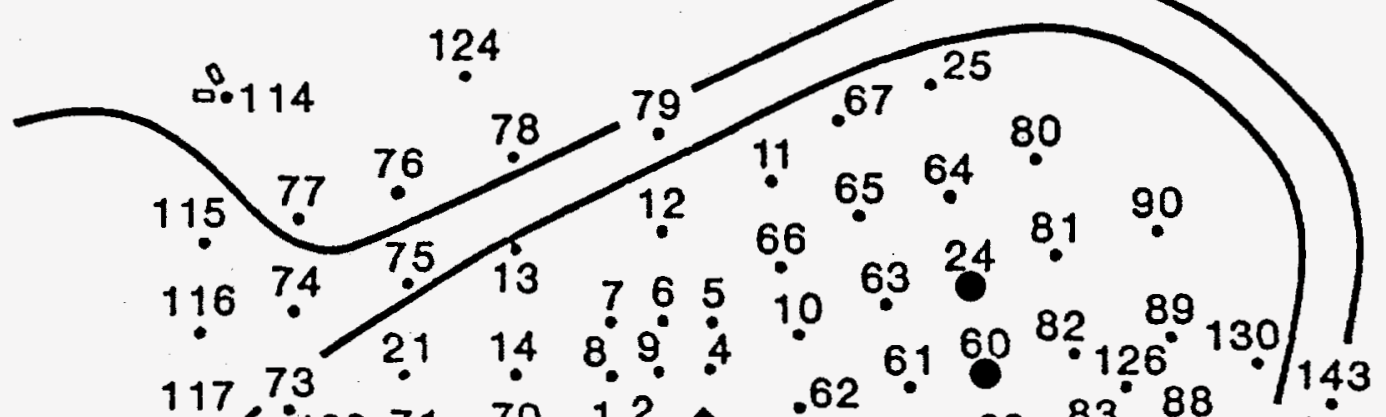

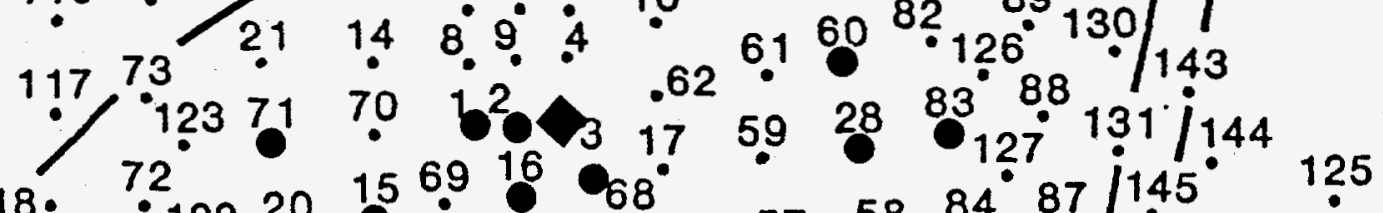

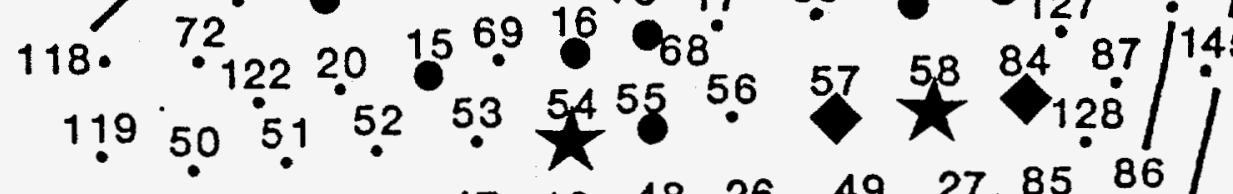

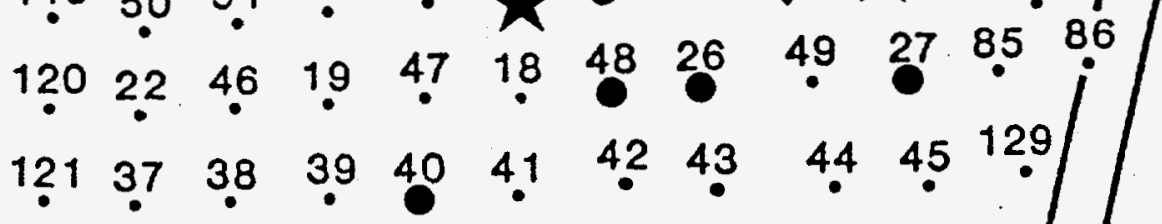

$\begin{array}{lllllllllll}91 & 23 & 36 & 29 & 35 & 30 & 34 & 31 & 33 & 32\end{array}$

$92 \quad 93 \quad 94 \quad 95 \quad 96 \quad 9798$

10610510410310210110099

$107 \quad 108 \quad 109 \quad 110 \quad 111 \quad 112 \quad 113$

$133 \quad 134135136137$

142141140139138

1

1 SYMBOL RANGE (ppb)

\section{LEGEND}

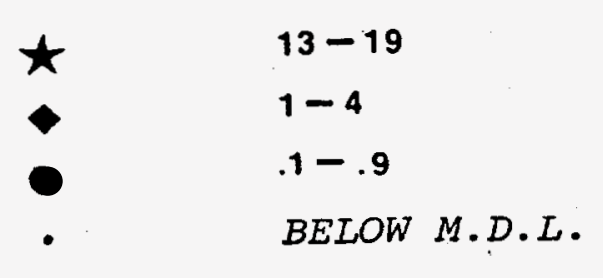

MISCELLANEOUS

CHEMICAL BASIN

TRICHLOROMETHANE

S.R.P.

NOVEMBER, 1986 


\section{FIGURE 48}

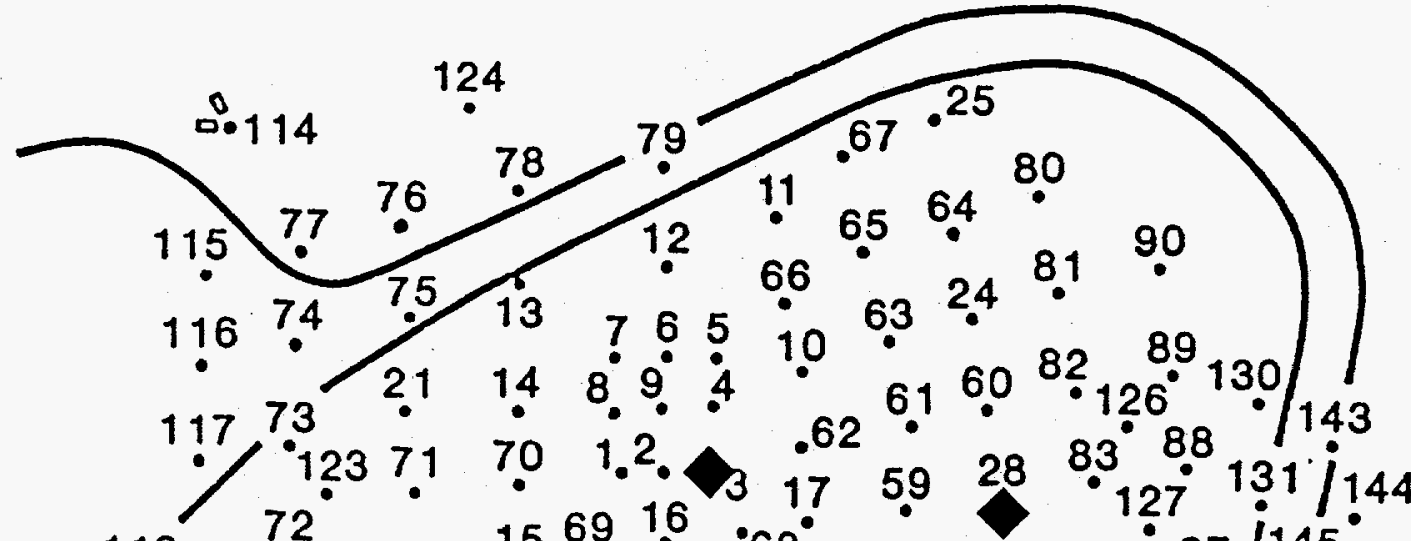

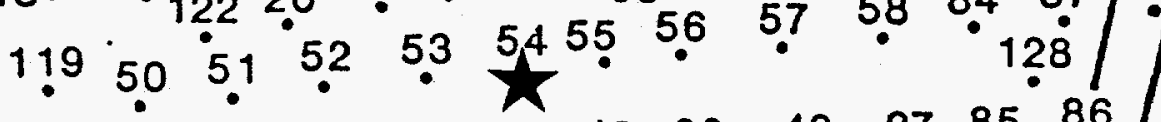

$\begin{array}{llllllllllll}120 & 22 & 46 & 19 & 47 & 18 & 48 & 26 & 49 & 27 & 85 & 86\end{array}$

$\begin{array}{lllllllllll}121 & 37 & 38 & 39 & 40 & 4.1 & 42 & 43 & 44 & 45 & 129\end{array}$

$\begin{array}{llllllllll}9 . & 23 & 36 & 29 & 35 & 30 & 34 & 3.1 & 33 & 32\end{array}$

$\begin{array}{llllllll}92 & 93 & 9.4 & 95 & 96 & 97 & 98\end{array}$

10610510410310210110099

$\begin{array}{lllllllll}107 & 108 & 109 & 110 & 111 & 112 & 113\end{array}$

$\begin{array}{lllll}133 & 134 & 135 & 136 & 137\end{array}$

142141140139138

]

]

1

]

]

LEGEND

SYMBOL RANGE (ppb)

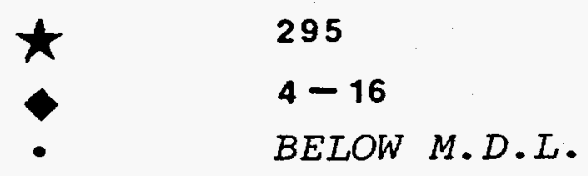

MISCELLANEOUS

$\begin{array}{ll}\star & 295 \\ & 4-16 \\ \text { BELOW M.D.L. }\end{array}$


FIGURE 49

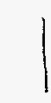

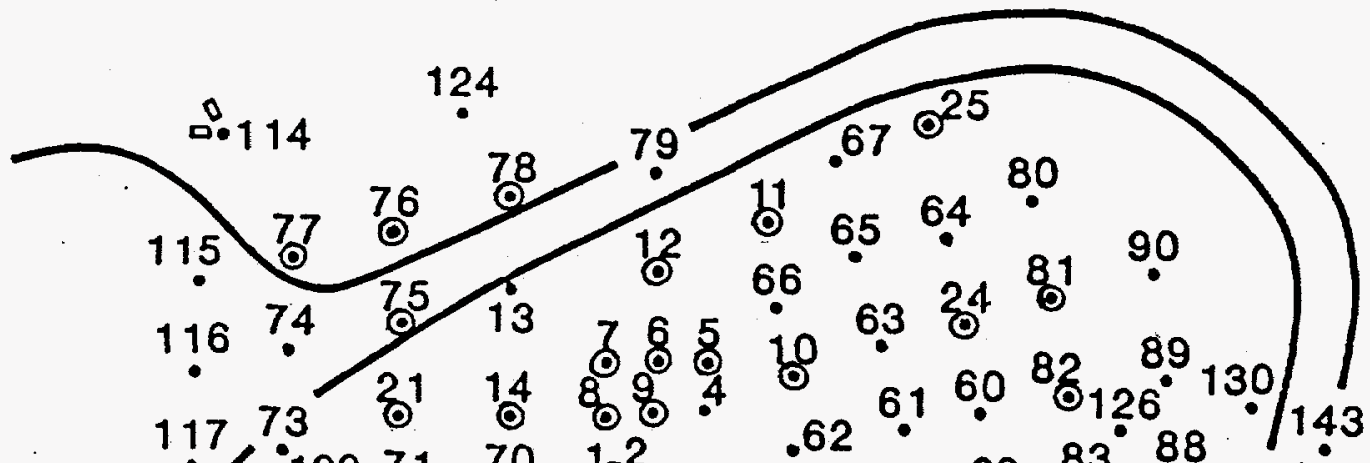

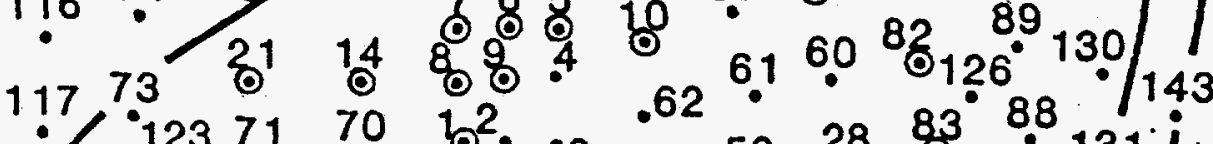

132

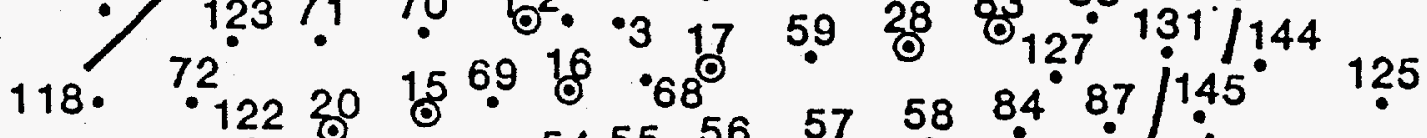

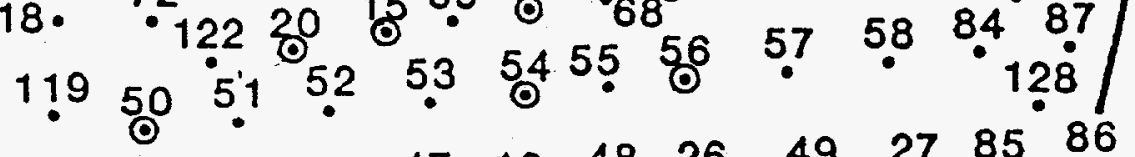

\begin{tabular}{llllllllllll}
120 & 32 & 46 & 19 & 47 & 18 & 48 & 26 & 49 & 27 & 85 & 86 \\
\hline & 0 & 0 & 0 & 0 & 0 & 0 & 0 & 0
\end{tabular}

$\begin{array}{lllllllllll}121 & 37 & 38 & 3.9 & 40 & 4.1 & 42 & 43 & 4.4 & 45 & 129\end{array}$

$\begin{array}{llllllllll}91 & 23 & 36 & 29 & 35 & 30 & 34 & 31 & 33 & 32\end{array}$

$\begin{array}{llllllll}92 & 9.3 & 94 & 95 & 96 & 97 & 98\end{array}$

]

10610510410310210110099

107108 109 $110111 \quad 112113$

$133 \quad 134 \quad 135136137$

142141140139138

1

]

]

1 SYMBOL RANGE (ppb)

]

○

\section{LEGEND}

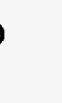

\footnotetext{
2- 48

BELOW M.D.L.
}

\author{
MISCELLANEOUS \\ CHEMICAL BASIN \\ TRANS 1, 2 \\ DICHLOROETHYLENE \\ S.R.P.
}

$02550 \mathrm{ft}$.

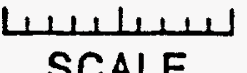
SCALE 
FIGURE 50

GASOLINE RANGE HYDROCARBONS ${ }^{\backslash}$

10ppb STANDARD CHROMATOGRAM

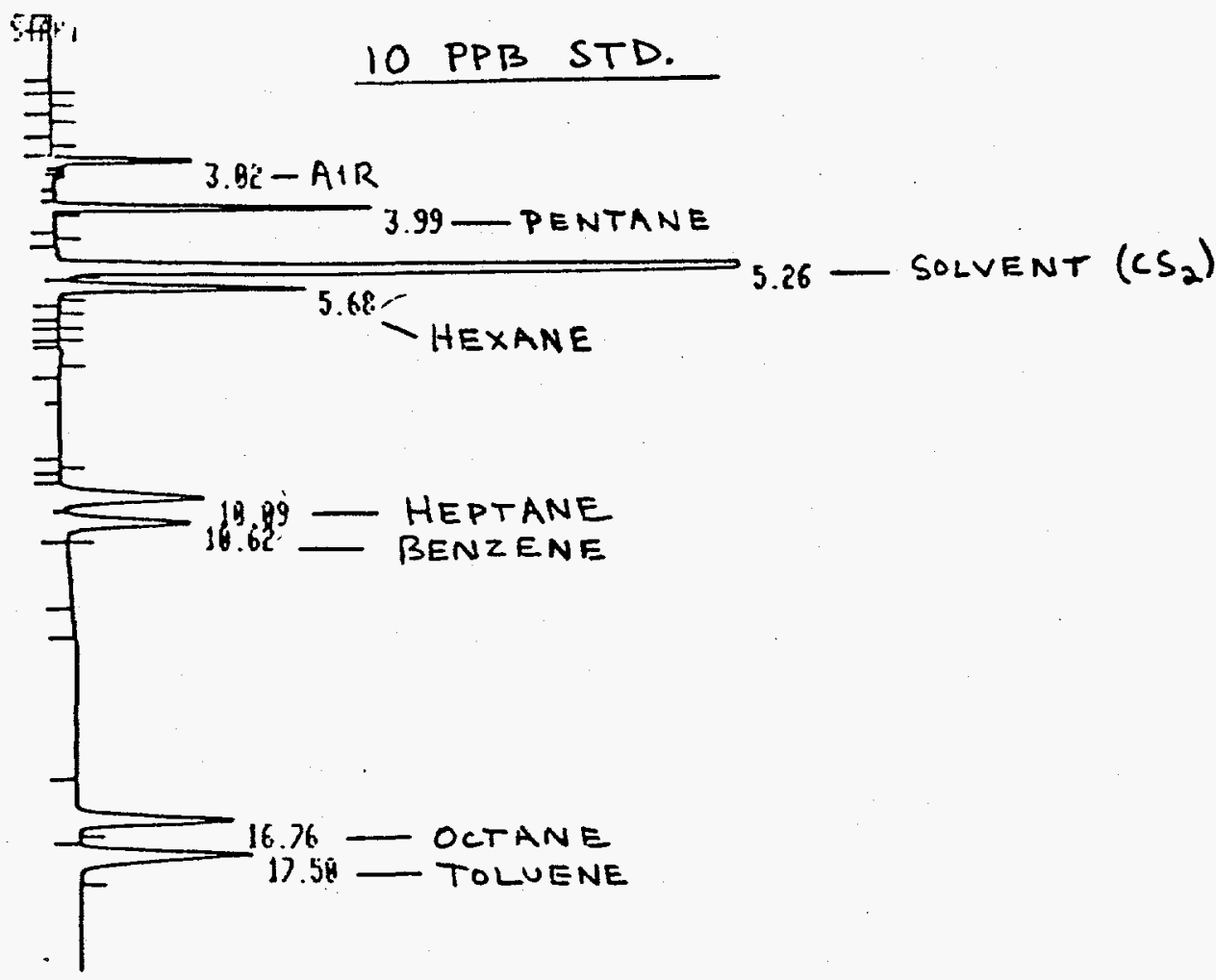

\section{KUN 89}

WUK'KF ILE ID : $B$

$007 / 14 / 86 \quad 22: 48: 46$

MOKKFILE NAME:

\begin{tabular}{|c|c|c|c|c|c|}
\hline PENTANE & $\begin{array}{r}\text { AREA\% } \\
\text { Ri } \\
3.82 \\
3.99\end{array}$ & $\begin{array}{r}\text { AREA } \\
18963 \\
22619\end{array}$ & $\begin{array}{r}\text { TYFE } \\
\text { PY } \\
\text { PB }\end{array}$ & $\begin{array}{l}\text { AR/HT } \\
0.112 \\
0.191\end{array}$ & $\begin{array}{l}\text { AREA\% } \\
1.944 \\
4.803\end{array}$ \\
\hline HEXANE & $\begin{array}{l}5.66 \\
5.68\end{array}$ & $\begin{array}{r}42284 \theta \\
21494\end{array}$ & $\begin{array}{l}\mathrm{PB} \\
\mathrm{BB}\end{array}$ & $\begin{array}{l}0.115 \\
0.128 \\
\end{array}$ & $\begin{array}{r}74.823 \\
3.883\end{array}$ \\
\hline HEPTANE & 18.09 & 22003 & PY & 0.219 & 3.894 \\
\hline BENZENE & 18.62 & 17559 & YB & 0.197 & 3.167 \\
\hline OCTAINE & 16.06 & 19599 & $\underline{P B}$ & 0.181 & 3.468 \\
\hline TOLUENE & 17.58 & 28045 & $\overline{\mathrm{B}} \overline{\mathrm{B}}$ & $0.2 \overline{32}$ & 4.963 \\
\hline
\end{tabular}

IOTAL AREA $=\quad 565120$

HUL FACTOR $=1.00 \theta 6 E+B \theta$ 
FIGURE 51

GASOLDNE RANGE HYDROCARBONS

5Ppb STANDARD GHROMATOGRAM

5 PPB STD.

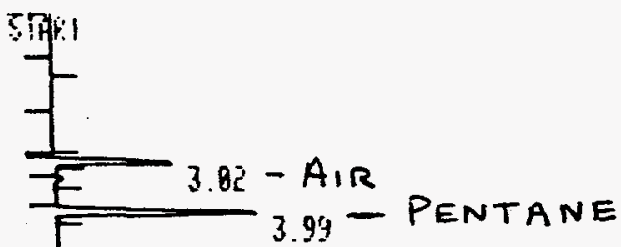

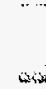

1

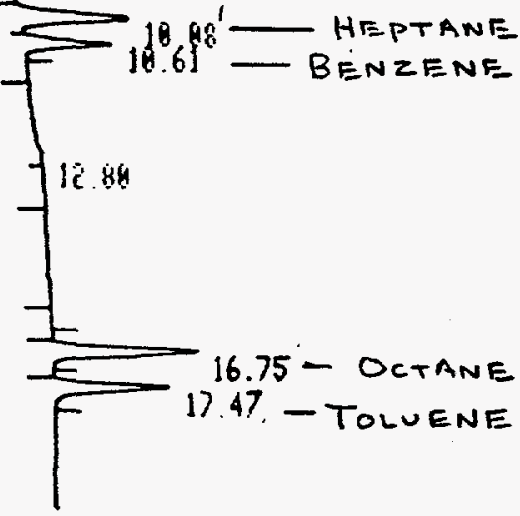

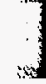

RUH 91

OLT $T / 14 / 86 \quad 23: 48: 51$

HIKKF HLE ID: E

WUKKF ILE HAHE:

Akit A\%

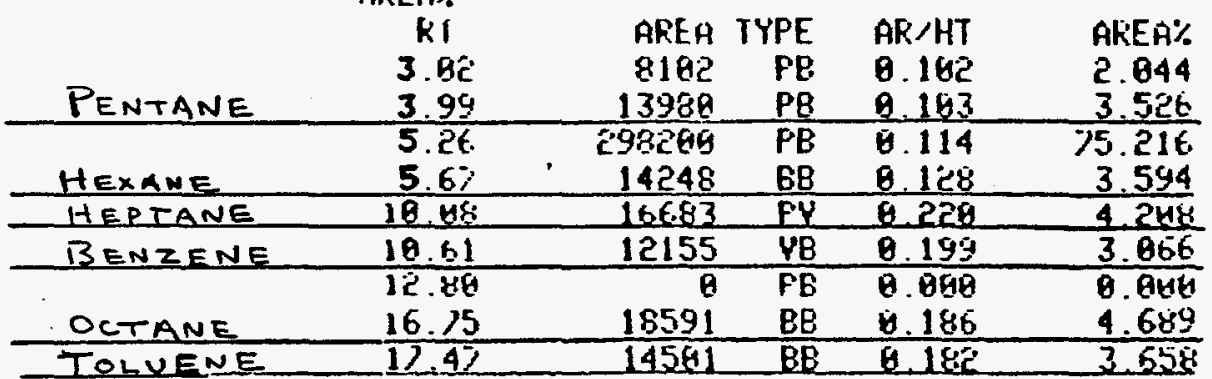

